\title{
USE OF TOPOGRAPHIC LIDAR POINT CLOUDS FOR BUILDING RECONSTRUCTION
}

\author{
by
}

Yipeng Yuan

B.A., Computer Science, York University, Toronto, Ontario, Canada, 2003

\author{
A thesis \\ presented to Ryerson University \\ in partial fulfillment of the \\ requirements for the degree of \\ Master of Applied Science \\ in the Program of Civil Engineering
}

Toronto, Ontario, Canada

CYipeng Yuan, 2009 


\section{DECLARATION}

I hereby declare that I am the sole author of this thesis.

I authorize Ryerson University to lend this thesis to other institutions or individuals for the purpose of scholarly research.

(Signature above)

Yipeng Yuan

I further authorize Ryerson University to reproduce this thesis by photocopying or by other means, in total or in part, at the request of other institutions or individuals for the purpose of scholarly research.

(Signature above)

Yipeng Yuan 


\section{BORROWER'S PAGE}

Ryerson University requires the signatures of all persons using or photocopying this thesis.

Please sign below, and give address and date.

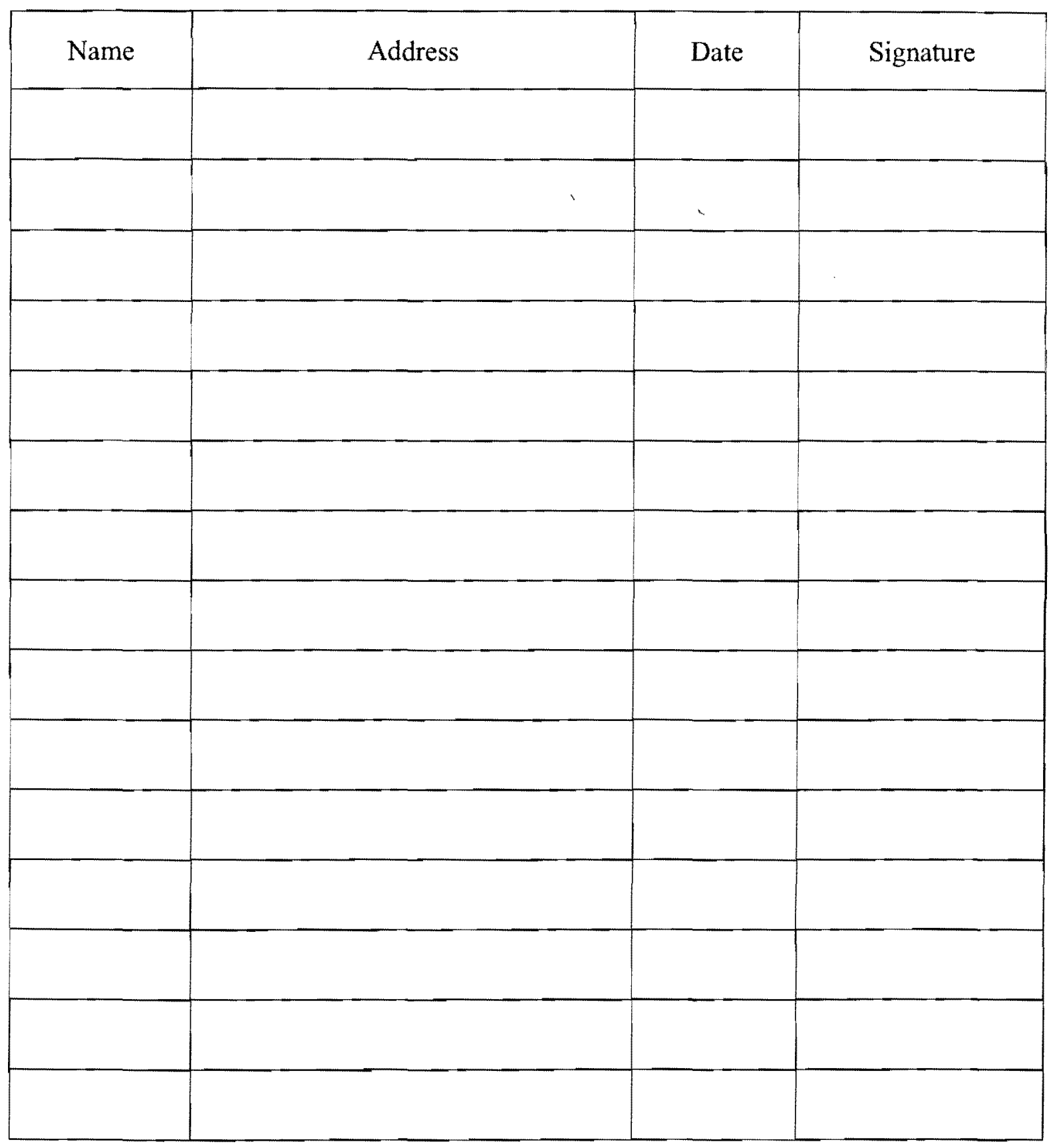




\section{USE OF TOPOGRAPHIC LIDAR POINT CLOUDS FOR BUILDING RECONSTRUCTION}

Yipeng Yuan

Master of Applied Science, Ryerson University, Toronto, Ontario, Canada, 2009

\section{ABSTRACT}

Demand for three-dimensional (3D) urban models keeps growing in various civil and military applications. Topographic LiDAR systems are capable of acquiring elevation data directly over terrain features. However, the task of creating a large-scale virtual environment still remains a time-consuming and manual work. In this thesis a method for 3D building reconstruction, consisting of building roof detection, roof outline extraction and regularization, and $3 \mathrm{D}$ building model generation, directly from LiDAR point clouds is developed. In the proposed approach, a new algorithm called Gaussian Markov Random Field (GMRF) and Markov Chain Monte Carlo (MCMC) is used to segment point clouds for building roof detection. The modified convex hull $(\mathrm{MCH})$ algorithm is used for the extraction of roof outlines followed by the regularization of the extracted outlines using the modified hierarchical regularization algorithm. Finally, 3D building models are generated in an ArcGIS environment. The results obtained demonstrate the effectiveness and satisfactory accuracy of the developed method. 


\section{ACKNOWLEDGEMENTS}

I would like to express my gratitude and appreciation to my supervisors, Professor Dr. Jonathan Li and Professor Dr. Michael A. Chapman for guiding me throughout my work, for their continuous and generous help and support. Their valuable advice and comments are exceptionally precious for me.

Many thanks to the members of examining committee, Professor Dr. Songnian Li, Professor Dr. Ahmad Shaker for reviewing and commenting my thesis.

Special thanks go to $\mathrm{Yu} \mathrm{Li}$ for his encouragement and great help in my study. Time spent with him is rewarding and appreciated. I also acknowledge the support from my fellow graduate students, Xiangyu Si, Wenxia Tan, Yao Lu, Xian Guan and Faranak Amirsalari.

I would like to thank Tao Cui, $\mathrm{Xu}$ Lin and Yanrong Liu for their sincere friendship and encouragement during the hardest time while completing my thesis.

My deepest appreciation goes to my parents, my sisters and their families. Without their unconditional love and faith, I could not have achieved success in my career. 


\section{TO MY PARENTS}




\section{TABLE OF CONTENTS}

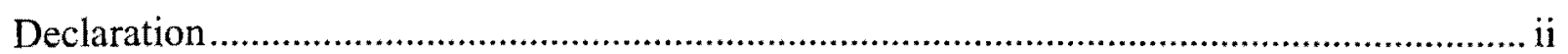

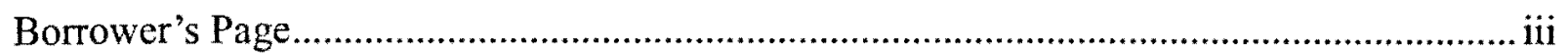

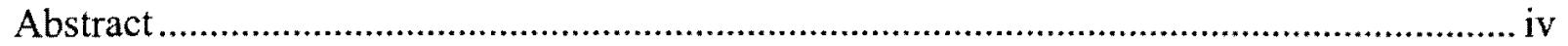

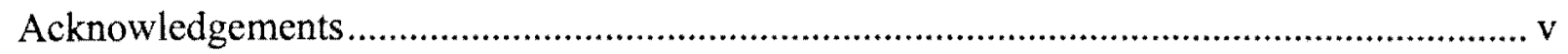

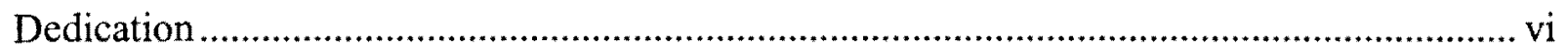

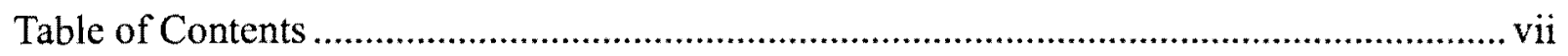

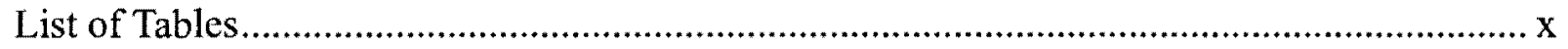

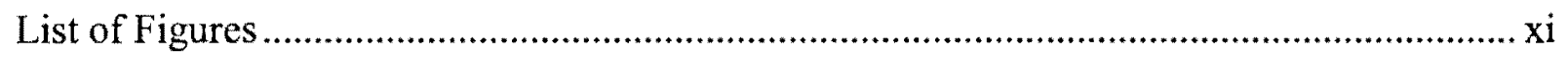

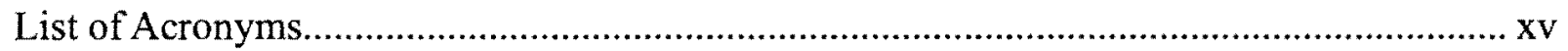

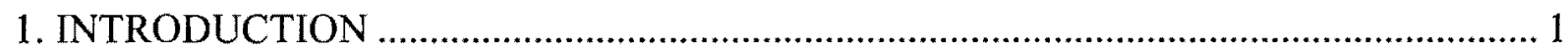

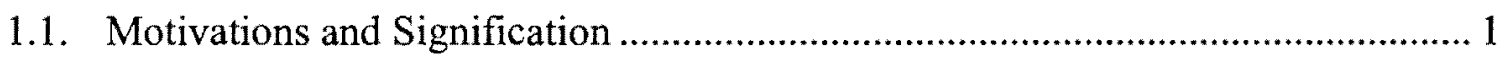

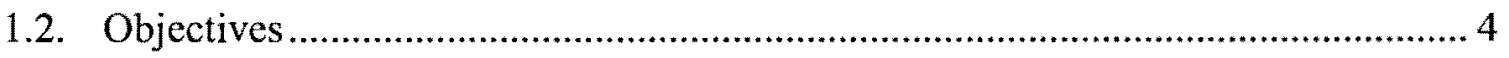

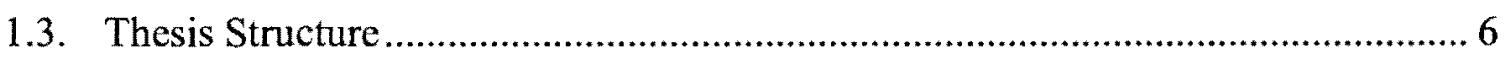

2. TOPOGRAPHIC LIDAR FOR 3D CITY MODELING: AN OVERVIEW ....................... 8

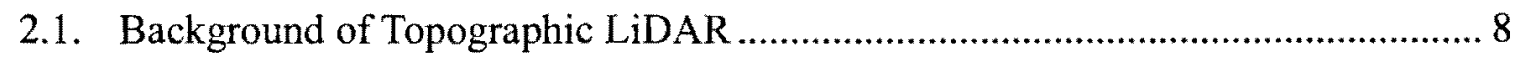

2.2. Working Principles of Topographic LiDAR .......................................................... 10

2.3. Summary of Main Topographic LiDAR Systems ............................................... 15

2.4. Basic Ranging Formulas ............................................................................. 17 
2.5. Segmentation of Topographic LiDAR Point Clouds........................................ 20

2.6. Building Outline Extraction and Regularization ................................................ 26

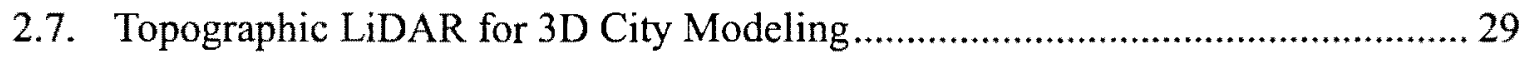

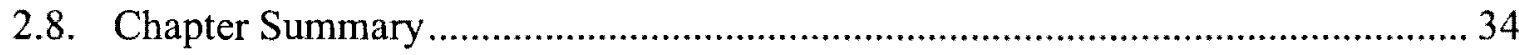

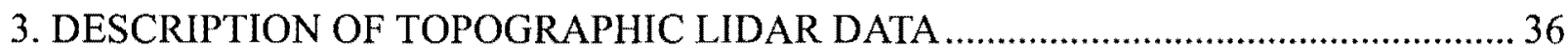

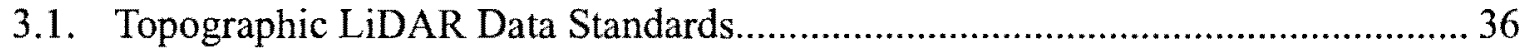

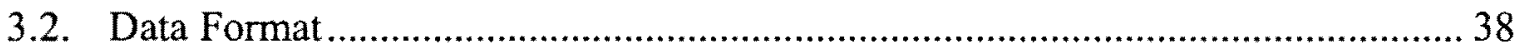

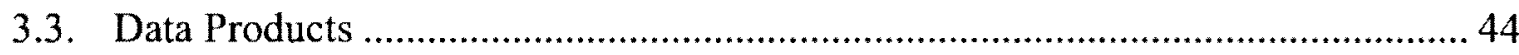

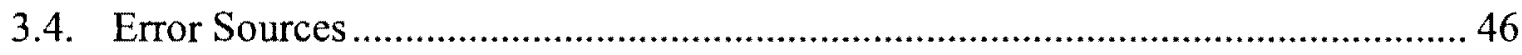

3.5. Data Ground Processing ..................................................................................... 51

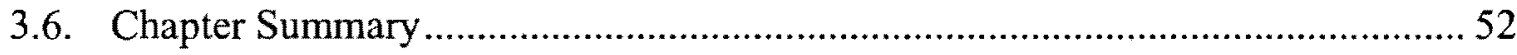

4. METHODOLOGY FOR BUILDING RECONSTRUCTION …...................................5

4.1. Segmentation Algorithm for Building Roof Detection ......................................5 54

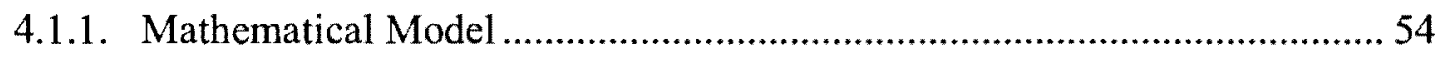

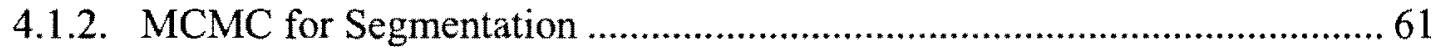

4.2. Building Outline Extraction and Regularization Algorithms ................................64

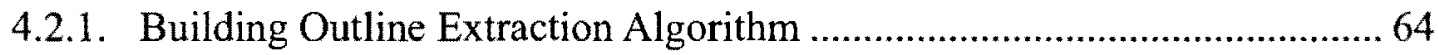

4.2.2. Building Outline Regularization Algorithm...............................................6 68

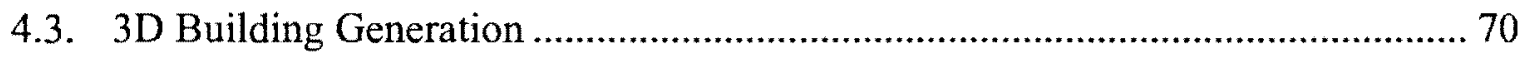

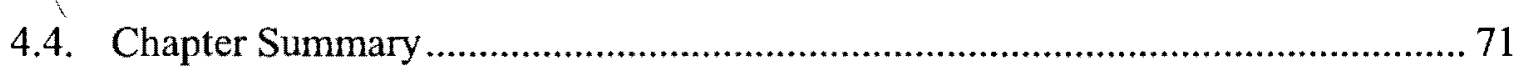




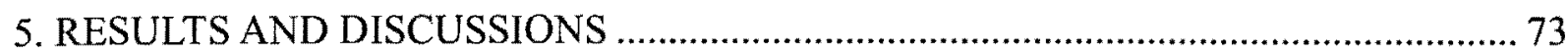

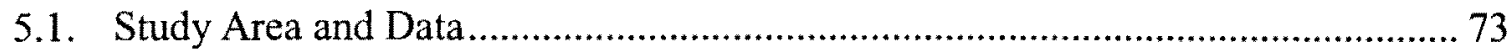

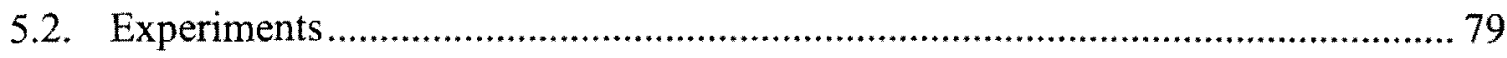

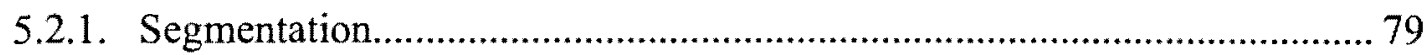

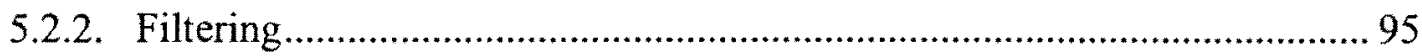

5.2.3. 3D Building Reconstruction in ArcGIS .............................................. 100

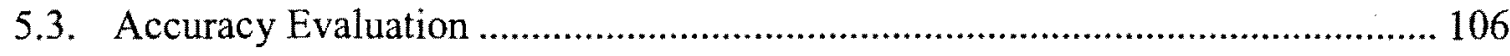

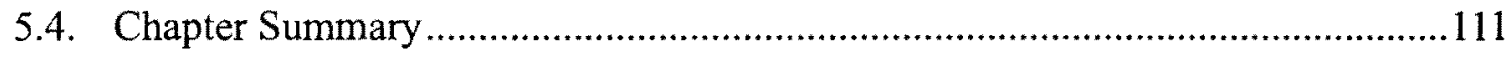

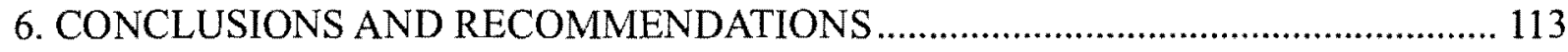

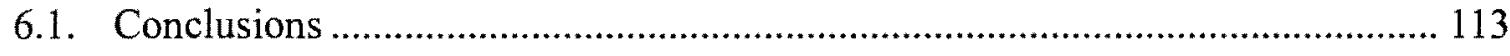

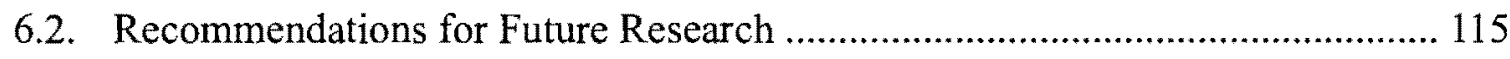

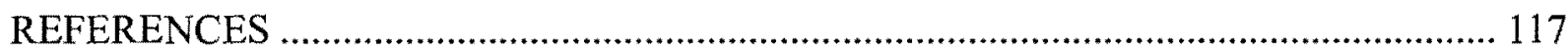




\section{LIST OF TABLES}

Table 2.1 Main manufacturers of topographic LiDAR systems

Table 2.2 Comparison between topographic LiDAR and photogrammetry

Table 3.1 Existing common LiDAR data file formats 40

Table 5.1 Topographic LiDAR data specifications 74

Table 5.2 Aerial image specification 76

Table 5.3 Sample building properties

Table 5.4 Input constants 80

Table 5.5 Parameters of sample buildings 81

Table 5.6 Means and their colour representations. 91

Table 5.7 Building roof outline extraction and regularization 99

Table 5.8 Building height estimation 104

Table 5.9 Shapefiles and their area coverage 108

Table 5.10 Accuracy evaluation 109 


\section{LIST OF FIGURES}

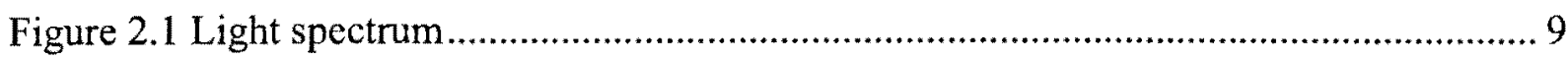

Figure 2.2 Paradigm of topographic LiDAR …............................................................ 10

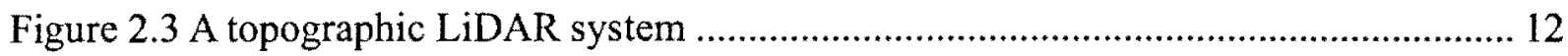

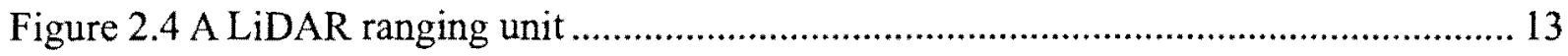

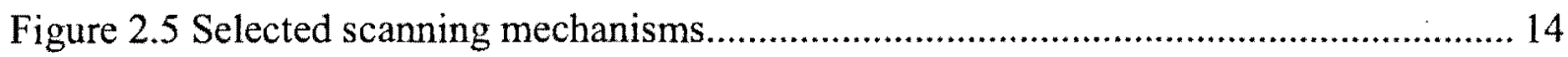

Figure 3.1 Sample topographic LiDAR data in ASCII format ............................................. 41

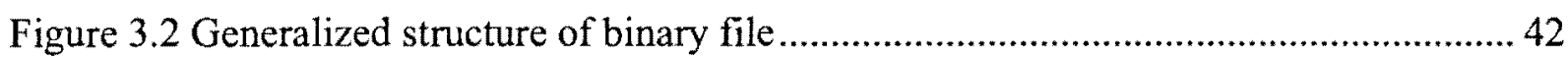

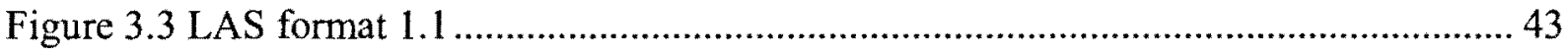

Figure 3.4 Error components of topographic LiDAR data ..................................................46

Figure 3.5 Illustrations of the results of misalignments.......................................................... 48

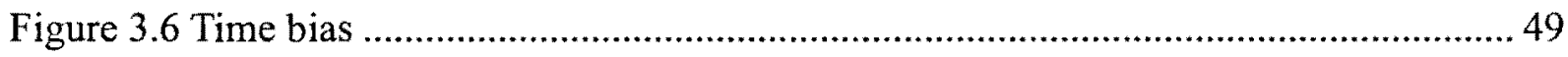

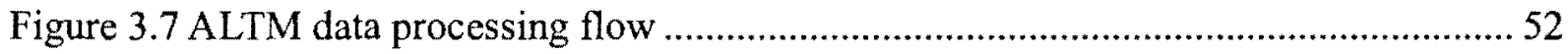

Figure 4.1 Relationship between label field and data field..................................................56

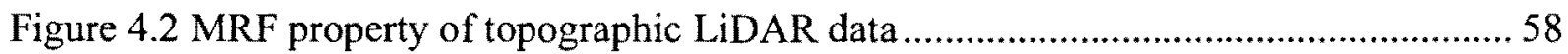

Figure 4.3 GMRF parameters and their interrelationships …............................................... 61

Figure 4.4 Flowchart illustrating Metropolis-Hastings algorithm ........................................63

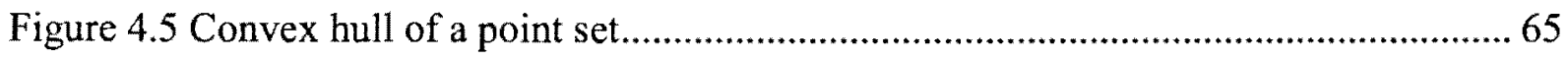

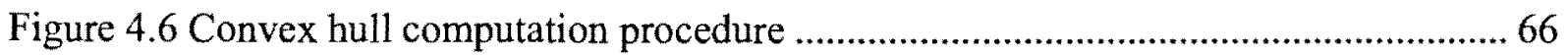


Figure 4.7 Regular concave shaped buildings

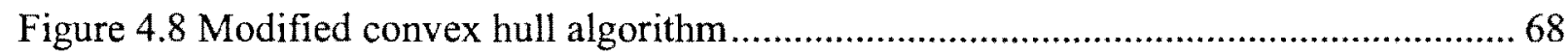

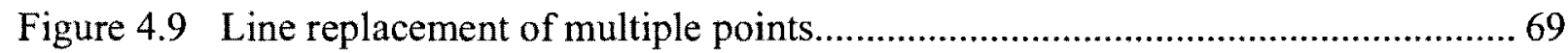

Figure 4.10 Process of 3D building generation in ArcGIS ............................................. 71

Figure 5.1 Topographic LiDAR point clouds of the University of Waterloo campus and surrounding area.. 73

Figure 5.2 Reference map to topographic LiDAR image. 74

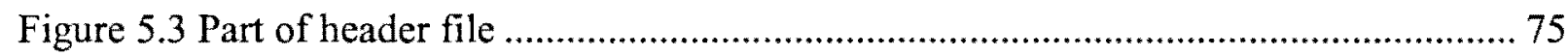

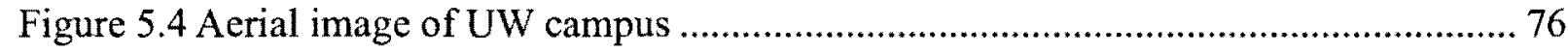

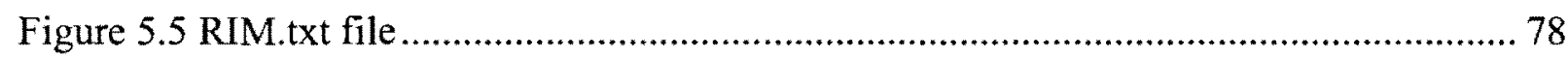

Figure 5.6 Topographic LiDAR Images of sample buildings .............................................. 79

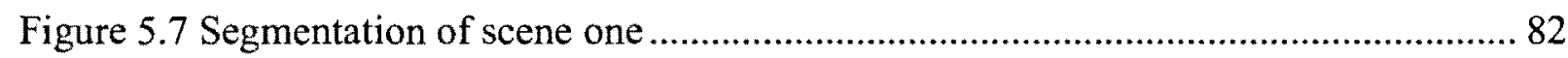

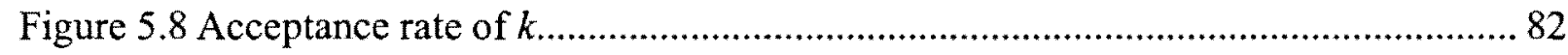

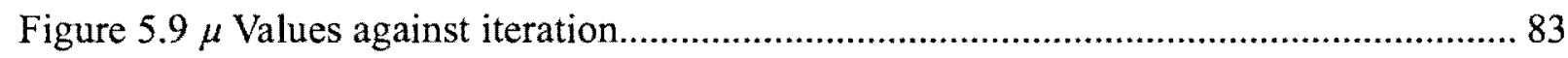

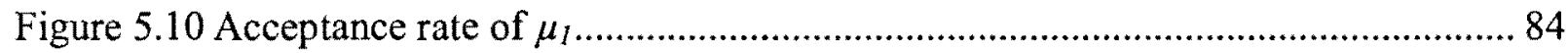

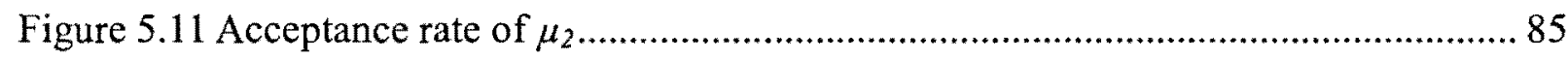

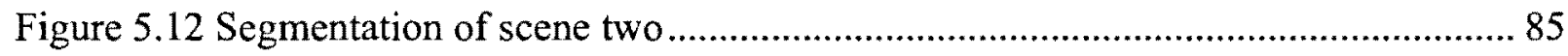

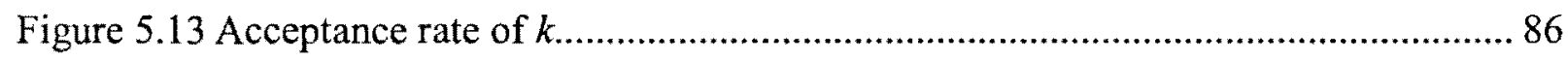

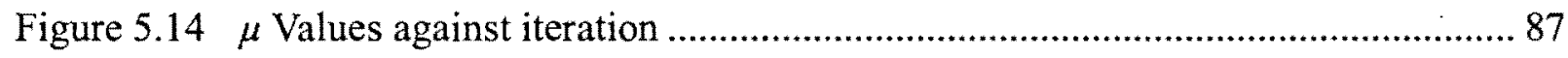

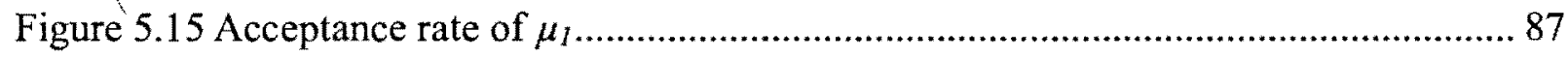




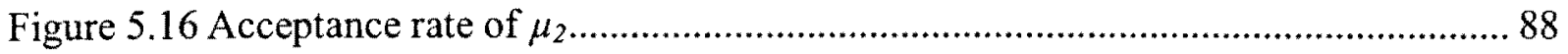

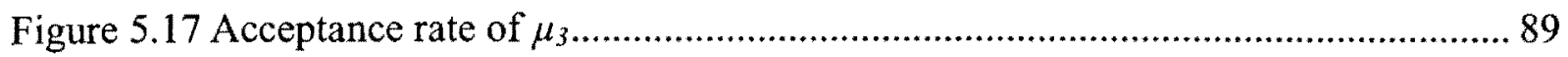

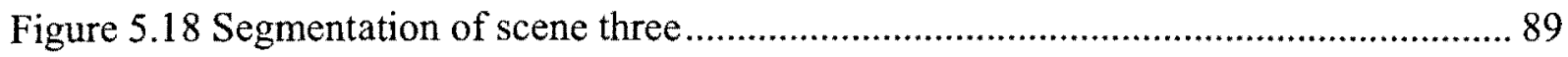

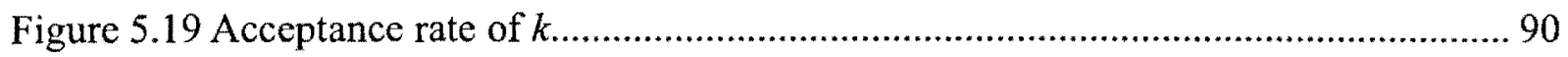

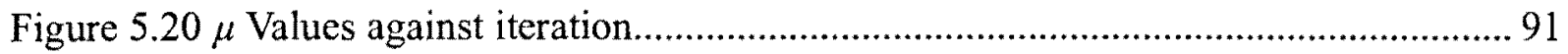

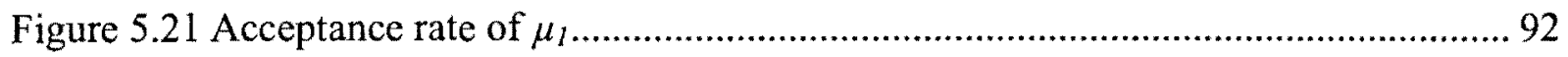

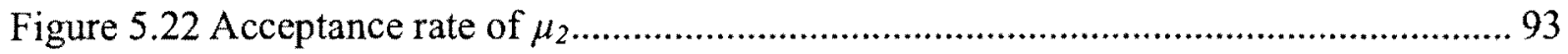

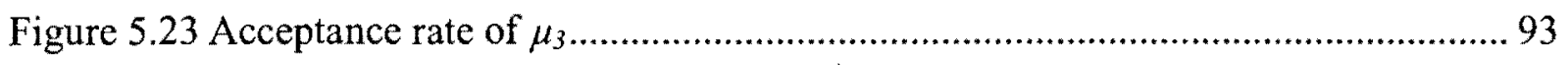

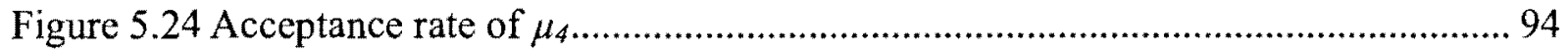

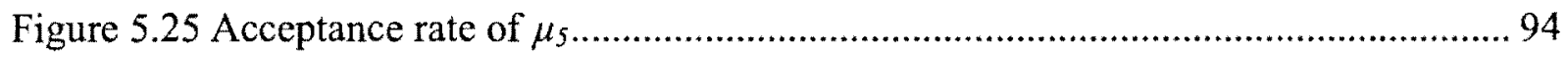

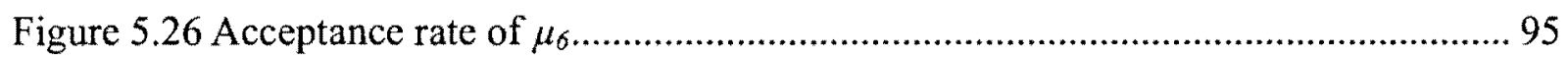

Figure 5.27 Optometry building and its neighboring HRV ................................................ 96

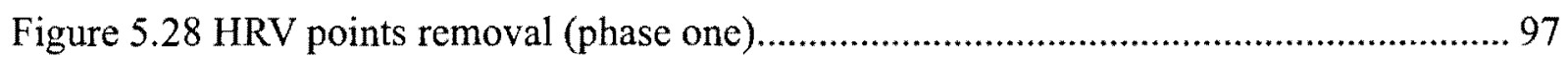

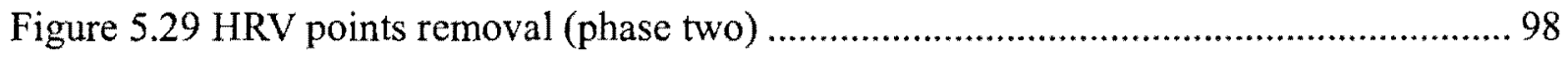

Figure 5.30 File re-formatting of RIM building roof outline points................................. 100

Figure 5.31 RIM building line coverage file in ArcGIS ................................................... 101

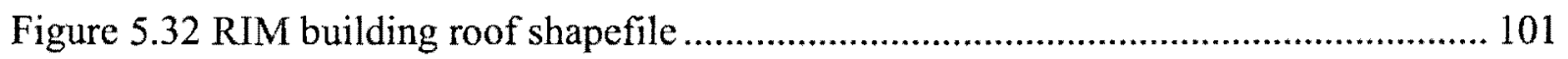

Figure 5.33 Shapefiles of three selected buildings .......................................................... 102

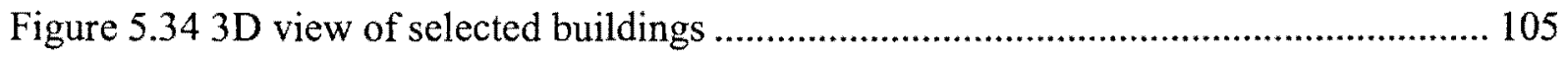

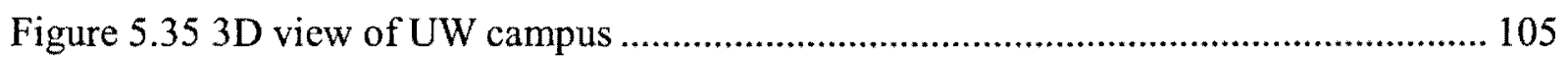


Figure 5.36 Overlay of shapefiles.

Figure 5.37 Overlay of RIM building roof points from topographic LiDAR data and standard shapefile 


\section{LIST OF ACRONYMS}

\begin{tabular}{|c|c|}
\hline AGL & Above Ground Level \\
\hline AMSL & Above Mean Sea Level \\
\hline ANSI & American National Standards Institute \\
\hline $\mathrm{AOI}$ & Area Of Interest \\
\hline $\mathrm{AR}$ & Acceptance Rate \\
\hline ASCII & American Standard Code for Information Interchange \\
\hline ASPRS & American Society for Photogrammetry and Remote Sensing \\
\hline $\mathrm{CE}$ & Commission Error \\
\hline $\mathrm{CW}$ & Continuous Wave \\
\hline DEM & Digital Elevation Model \\
\hline DGPS & Differential Global Positioning System \\
\hline DSM & Digital Surface Model \\
\hline DTM & Digital Terrain Model \\
\hline FR & Phase Ranging \\
\hline GIS & Geospatial Information System \\
\hline GMRF & Gaussian Markov Random Field \\
\hline GPS & Global Positioning System \\
\hline HRV & High Rise Vegetation \\
\hline
\end{tabular}




\begin{tabular}{|c|c|}
\hline IFOV & Instantaneous Field Of View \\
\hline IMU & Inertial Measurement Unit \\
\hline INS & Inertial Navigation System \\
\hline ISO & The International Standardization Organization \\
\hline ISWG & IEEE Committee on Earth Observations Standards Working Group \\
\hline LiDAR & Light Detection And Ranging \\
\hline LRV & Low Rise Vegetation \\
\hline $\mathrm{MCH}$ & Modified Convex Hull \\
\hline $\mathrm{MCMC}$ & Markov Chain Monte Carlo \\
\hline MMSE & Minimum Mean Square Estimate \\
\hline NDVI & Normalized Difference Vegetation Index \\
\hline $\mathrm{OA}$ & Overall Accuracy \\
\hline $\mathrm{OE}$ & Omission Error \\
\hline POS & Position and Orientation System \\
\hline PR & Pulse Ranging \\
\hline UTM & Universal Transverse Mercator \\
\hline UW & University of Waterloo \\
\hline WGS & World Geodetic System \\
\hline TIN & Triangulated Irregular Network \\
\hline
\end{tabular}




\section{INTRODUCTION}

Spatial information is gaining increasing attention from the general public, government agencies and commercial establishments. Topographic light detection and ranging (LiDAR), which integrates a laser scanner, a Global Positional System (GPS) receiver and an Inertial Measurement Unit (IMU), is capable of acquiring dense elevation point data over terrain rapidly, economically and accurately. Since the appearance of topographic LiDAR in 1990s, its applications extend from Digital Elevation Model (DEM) generation (Lee and Younan, 2003; Shan and Sampath, 2005), urban modeling (Haala and Brenner, 1997; Schwalbe et al., 2005) to disaster management (Webster et al., 2004; Dash et al., 2004). Topographic LiDAR has grown to a viable and valuable tool in the survey's toolbox (Jonas and Byrne, 2003). In this chapter, the motivations and the objectives of the study are illustrated, the structure of the thesis is summarized.

\subsection{Motivations and Signification}

Today more than half of the world's population lives in urban areas. As large cities around the globe keep propagating and become more populated, up-to-date geospatial information and three-dimensional (3D) city models are becoming increasingly important for applications such as city planning, crisis management, visualization, architecture, and landscaping (Tolt et al., 2007). 
Traditionally $3 \mathrm{D}$ city models are produced by conventional aerial photogrammetry or by semi-automated procedures for measurements in aerial imagery (Vosselman and Dijkman, 2001), in which the process is labour-intensive, time-consuming, expensive and error prone. Topographic LiDAR provides direct 3D information precisely over urban areas regardless of illumination conditions and reduces or eliminates the interpretation errors that may occur in traditional elevation data generation. These superior attributes make topographic LiDAR systems ideal for high-fidelity 3D urban model reconstruction. The scanning rate of modern topographic LiDAR systems is as high as $200 \mathrm{kHz}$ with the precision to centimeter level. As a result topographic LiDAR datasets are notoriously famous for their huge volume of data size. When a topographic LiDAR system is in operation, the data collected are non-selective. The system records everything under the flying route, which includes less wanted data like vegetation in metropolitan areas. In the past decade a wealth of research activities developed various approaches to extract topographic features from topographic LiDAR data. Several standardized workflows for the reconstruction of 3D city models exist, they are either based on photogrammetry or on LiDAR or on a combination of both data acquisition techniques. However, the automated reconstruction of reliable and highly accurate 3D city models is still a challenging task, requiring a workflow comprising several processing steps. Some of the most relevant challenges are building detection, building outline generation, building modeling, and last but not least, an accuracy assessment. 
Building detection focuses on locating point sets covering building roofs in the dataset and is achieved by using either segmentation or filtering methods. It is the most critical step in building reconstruction process and determines the quality of the building model. In the building outline extraction step, edge points forming the shape of the building roof arc traced out from building roof points. Due to the nature of topographic LiDAR, some of building roof edge points are missing, which makes building roof outlines distorted. The building shape regularization method removes distortions produced in the previous step. In the last step, 3D city models are reconstructed in a 3D environment. Commercial software tools for building modeling require, generally, a high degree of human interaction and most automated approaches described in literature stress the steps of such a workflow (Dorninger and Pfeifer, 2008).

As the demand for reconstruction of 3D building models keeps growing and high quality topographic LiDAR data become available, practical, integrated and efficient approaches are expected urgently. A greater number of research papers examine only one of aforementioned four aspects. For instance, Brovelli et al. (2002), Chen et al. (2007), Kilian et al. (1996), Kraus and Pfeifer (1998), Lee and Younan (2003), Sithole (2001), Vosselmann (2000) presented building detection approaches from gridded topographic LiDAR data. Huber et al. (2003) and Kim et al. (2006) depicted 3D building reconstruction methods with building roof shapes acquired from auxiliary data. Jwa et al. (2008) and Sampath and Shan (2007) 
illustrated the building outline extraction and building shape regularization methods from building point set of topographic LiDAR data. These partly finished algorithms may cause confusion and contradiction when choosing proper combination of approaches to achieve 3D applications. At the same time, few researchers presented the complete methodology including above four aspects for 3D city modeling.

The work demonstrated in this study is motivated by presenting an approach for generation of 3D city models from topographic LiDAR point clouds, which comprises the entire sequence from building detection, extraction and regularization to reconstruction. The proposed approach works on topographic LiDAR point cloud directly without an interpolation process and does not need maps, construction plan or GIS data to establish building roof shape. This approach has a substantial advantage over previous methods as the 3D building models are created solely from topographic LiDAR data. The algorithm is versatile in term of data resolution and, is applicable for topographic LiDAR data with a density around two points per square meter. However, it is capable of processing topographic LiDAR data with higher point density,

\subsection{Objectives}

The objective of this study is to develop a methodology for the generation of $3 \mathrm{D}$ building models from topographic LiDAR point cloud data. The proposed approach consists of five 
parts: building detection, roof outline extraction, building shape regularization, 3D building model creation and accuracy assessment. The methodology is implemented using the following four steps:

1. Point cloud segmentation.

2. Building roof outline extraction.

3. Roof outline regularization.

4. 3D building reconstruction in a GIS environment.

First, this study attempts an overview of the working principles of topographic LiDAR, physical components, data formats and various data processing algorithms, which offers the background for further study on point cloud segmentation and building reconstruction.

Second, this study attempts an extension of the literature to the utilization of Gaussian Markov Random Field (GMRF) and Markov Chain Monte Carlo (MCMC) algorithm for the segmentation of point clouds. The advantages of the algorithm include:

1. The GMRF-MCMC algorithm works directly on topographic LiDAR raw data without any data conversion.

2. The GMRF-MCMC algorithm does not require data pre-processing or support data. The segmentation algorithm achieves satisfactory results based on an accuracy analysis of sample data. 
Third, this study verifies building outline extraction and regularization algorithms from other researchers. Future studies will benefit in term of choosing proper matching methods with respect to the outcome from building extraction.

\subsection{Thesis Structure}

The thesis is comprised of six chapters.

Chapter 1 outlines the motivations of the study. Subsequently the study objectives are defined.

Chapter 2 provides a review of the working principle of topographic LiDAR systems and the methods used for building reconstruction from LiDAR data.

Chapter 3 describes the characteristics of topographic LiDAR data, various data formats, different levels of products, main error sources and data ground processing workflow.

Chapter 4 presents the methodology for building reconstruction. The GMRF-MCMC segmentation algorithm is described first, followed by the algorithms for building roof outline extraction and regularization. Finally the reconstruction of $3 \mathrm{D}$ building models is implemented in an ArcGIS environment. 
Chapter 5 reports the experimental results of building roof detection, roof outline extraction and regularization, and the generation of 3D building models in the GIS environment. The accuracy of the developed method is discussed.

Chapter 6 presents conclusions related to the developed method and suggests future work to extend the study. 


\section{TOPOGRAPHIC LIDAR FOR 3D CITY MODELING:}

\section{AN OVERVIEW}

As the name suggests, topographic LiDAR involves mounting a laser scanner on an aircraft or helicopter and setting it to scan the measurements of the surface along the flying route. In this chapter, some basic working principles and mathematical formulas are elaborated, then various algorithms on building extraction, building outline extraction and regularization and 3D city modeling will be examined and compared.

\subsection{Background of Topographic LiDAR}

Topographic LiDAR transmits laser beams to acquire elevation data of surface, its frequency is in the $500-1500 \mathrm{~nm}\left(0.5 \times 10^{-6} \mathrm{~m}-1.5 \times 10^{-6} \mathrm{~m}\right)$ range, with typical values of $1040-1060 \mathrm{~nm}$ (Baltsavias, 1999a). Figure 2.1 shows the composition of the light spectrum. The laser falls in the near infrared portion of the infrared region (red part close to visible light portion). According to the definitions from the European X-ray Laser Project, laser has three distinct aspects in contrast to other light sources:

1. Monochromatic. The light emitted from laser is monochromatic, which mean it only produces radiation of a specific wavelength, whereas visible light includes red, green and blue wavelengths (see Figure 2.1), and appears white/yellow when three wavelengths are added together. 
2. Coherence. Electromagnetic radiation can be regarded as a composition of individual wavetrains (fixed wavelength plus a length and a position). The individual wavetrains are extremely long for laser light, and adjacent wavetrains oscillate in synchronization manner, for visible light, the wavetrains are quite short.

3. Intensity and emittance. Laser beams can be very thin. As a result, a laser is usually extremely intense as it is concentrated on a tiny area. A laser beam does not diverge, which means it remains the "thin" state during its journey, so it is more focused than other light sources.

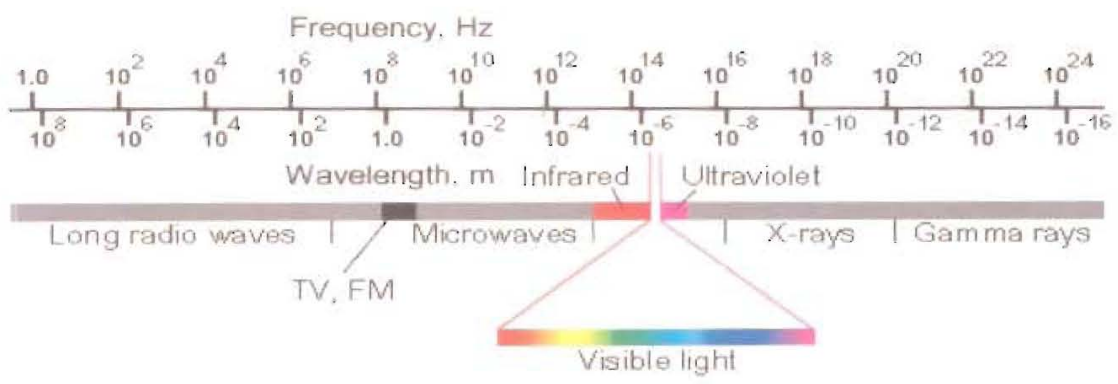

Figure 2.1 Light spectrum (Sentinel Archiving, Inc., 2008)

All the above important properties make the laser the excellent candidate of more accurate distance detection. So shortly after the advent of laser, very precise ranging was carried out with this new tool (Wehr and Lohr, 1999). LiDAR technology began to develop in late 1960 's, the first commercial topographic LiDAR mapping system became in 1993 (NOAA, 2008) thanks to the development of GPS and inertial navigation technologies. 
2. Coherence. Electromagnetic radiation can be regarded as a composition of individual wavetrains (fixed wavelength plus a length and a position). The individual wavetrains are extremely long for laser light, and adjacent wavetrains oscillate in synchronization manner, for visible light, the wavetrains are quite short.

3. Intensity and emittance. Laser beams can be very thin. As a result, a laser is usually extremely intense as it is concentrated on a tiny area. A laser beam does not diverge, which means it remains the "thin" state during its journey, so it is more focused than other light sources.

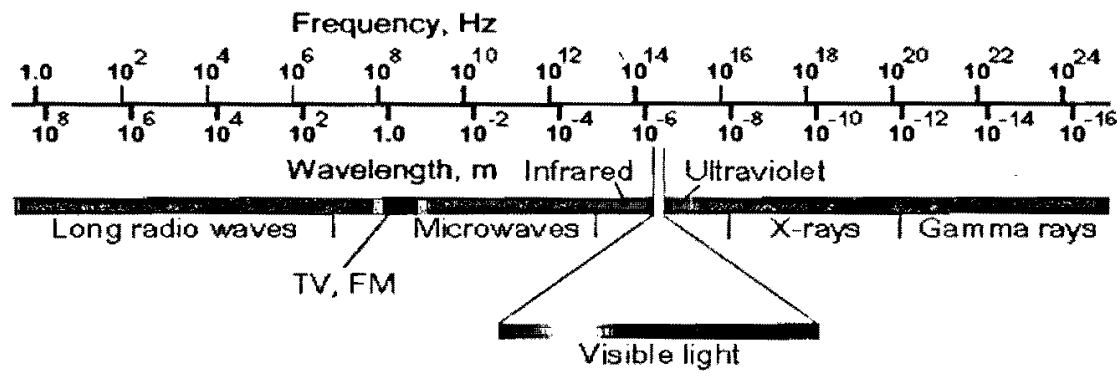

Figure 2.1 Light spectrum (Sentinel Archiving, Inc., 2008)

All the above important properties make the laser the excellent candidate of more accurate distance detection. So shortly after the advent of laser, very precise ranging was carried out with this new tool (Wehr and Lohr, 1999). LiDAR technology began to develop in late 1960's, the first commercial topographic LiDAR mapping system became in 1993 (NOAA, 2008) thanks to the development of GPS and inertial navigation technologies. 


\subsection{Working Principles of Topographic LiDAR}

Figure 2.2 demonstrates generic topographic LiDAR in operation. The preferred platforms of topographic LiDAR systems are fixed-wing aircrafts or helicopters. According to Baltsavias $(1999 \mathrm{c})$, helicopters cruise at $40-90 \mathrm{~km} / \mathrm{h}$ with a typical flying height of $200-300 \mathrm{~m}$, and are typically used in applications of small width, elongated areas (e.g., power lines, corridor mapping, topographic and bathymetric mapping along coastlines) or small areas (e.g., airports, open pit mines). They are also capable of conducting data capture when low speed (flood mapping) or high maneuverability (road mapping) is required. The fixed-wing aircrafts usually travel at $160-270 \mathrm{~km} / \mathrm{h}$ with altitude of $500-1000 \mathrm{~m}$, which can cover a larger area in a relatively short time.

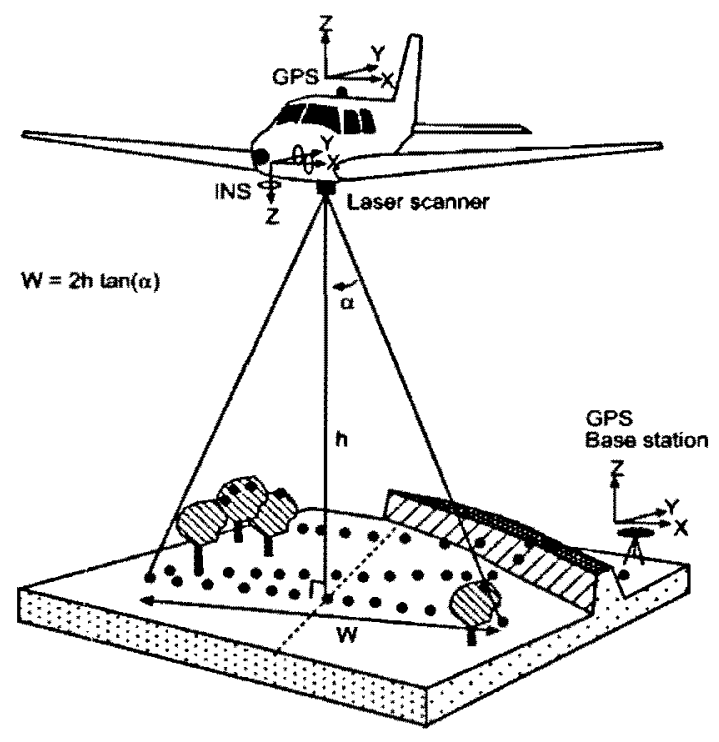

Figure 2.2 Paradigm of topographic LiDAR (Straatsma and Middelkoop, 2006)

When the aircraft flies over the target area, the scanner mounted emits LiDAR beams to the 
ground and, the scanning rate could be as high as $200 \mathrm{kHz} / \mathrm{s}$. Absorptions and reflections occur with atoms, molecules and aerosol which float in the air during the emitted LiDAR beam's travel to the ground. These changes could be recorded for atmosphere remote sensing, which is the scope of atmospheric science studies. For photogrammetric applications the interactions with man-made or natural features on the ground should be concentrated on. After the LiDAR beams hit the ground, the same phenomenon happens as it does in the air, part of LiDAR beams are back scattered to the air and are recoded. The velocity of light is constant, $299,792,458 \mathrm{~m} / \mathrm{s}$, and by calculating the difference between the time when LiDAR beams is generated and the time when its return is captured, the accurate elevation data of sampled points on the earth can be determined. The divergence of LiDAR beam is quite limited and the ground surface covered by single LiDAR beam is limited as well. By adding oscillating devices, LiDAR beams can scan the earth in a systemic way covering a wider area in one strip of flight.

Topographic LiDAR point cloud data, like images in photogrammetry, must be geo-referenced, so they can be utilized by other geo-spatial applications. On-board GPS device provides the geo-location of each sample point. Using ground GPS stations, high accuracy can be reached. INS device records aircraft orientation (i.e., attitude) data. Through the integration of LiDAR scanning data, GPS data and INS data, a densely sampled, geo-referenced yet highly precise elevation dataset is achieved. Millions of points are 
sampled in one dataset, sometimes referred to as a point cloud.

A complete topographic LiDAR system consists of several integrated parts. Flood and Gutelius (1997), Wehr and $\operatorname{Lohr}(1999)$ and Webster and Dias (2006) all analyzed the composition of topographic LiDAR systems. These researchers agreed that the three parts should be included in order to maintain its full functionality.

Figure 2.3 shows a complete topographic LiDAR system. According to Wehr and Lohr (1999), a typical topographic LiDAR system is comprised of three main parts: ranging unit, opto-mechanical scanner and control and processing unit. The ranging unit comprises the emitting LiDAR beam and the electro-optical receiver. The LiDAR scanner deflects a ranging beam in a certain pattern, causing it to move back and forth along the flying path, so that an object surface is sampled with a high point density. The control and processing unit consists of scanning control and monitor equipment, position and orientation system (POS) and on-board computers to record elevation and auxiliary data.

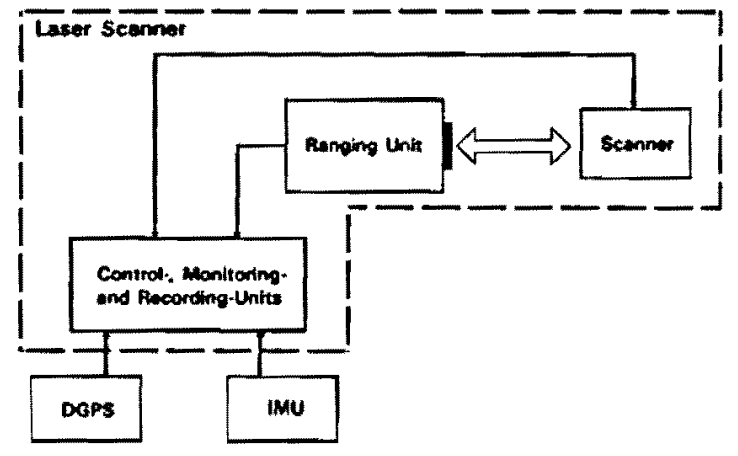

Figure 2.3 A topographic LiDAR system (Wehr and Lohr, 1999) 
Figure 2.4 displays the composition of a ranging unit. A pulse generator usually applies xenon flash tubes, arc lamps, metal-vapor lamps or semiconductors to generate the LiDAR beam. Through the aperture on the transmitter, the LiDAR beam is emitted to the ground, at the same time the counter is triggered. When the LiDAR beam hits the earth, a tiny part of surface is illuminated that is called a footprint. The smaller the footprint size, the higher the accuracy of the LiDAR range unit. When the echo of LiDAR beam is captured by the receiver, the counter is stopped, the accurate round trip time of LiDAR beam is documented. The intensity of echo may be recorded as well, which is the ratio to the emitted the LiDAR beam, and symbolized as a digital number.

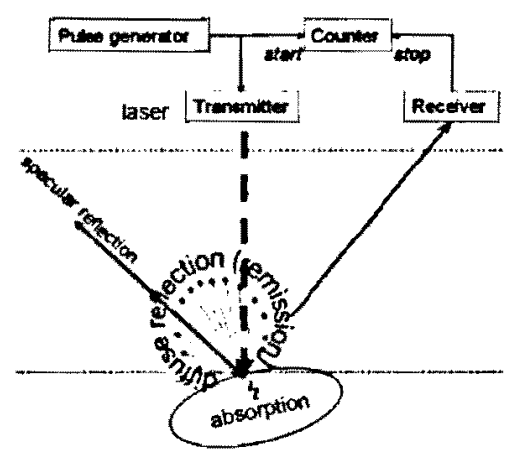

Figure 2.4 A LiDAR ranging unit (Pfeifer and Briese, 2007)

Since the divergence rate of LiDAR beam is so small, a scanner is introduced in order to cover the reasonable swath of the ground surface beneath the fly route. Figure 2.5 lists some of scanning mechanisms applied by scanners. An oscillating mirror usually produces Z-shaped lines and its scanning pattern is bidirectional. This type of systems involves stopping and accelerating when each scanning line is finished. The Palmer scan (mutating 
mirrors) results in elliptical pattern due to its inclining mirror design. The rotating polygon scanners (including its variation-multifaceted scanner) generate parallel lines and always start to scan from one direction. For a fiber scanner, an array of optical fibers is mounted in the focal plane of both transmitting and receiving lenses respectively. Two fiber arrays have identical number of fibers. Fibers on the transmitting side emit LiDAR beam to ground in sequence, fibers on the receiver side detect reflected LiDAR beam in sequence. No mechanical movement is involved during the set-up, which is distinct from the three above mentioned scanning patterns, so a higher scanning rate can be achieved.
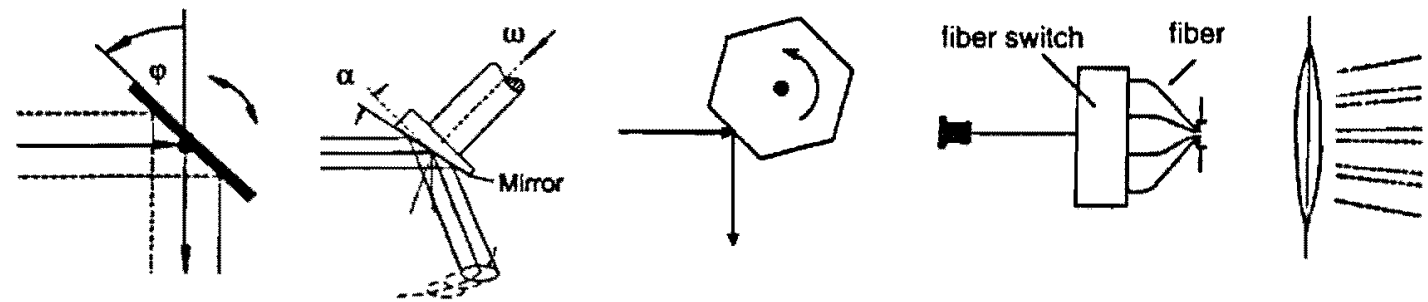

Figure 2.5 Selected scanning mechanisms (Wehr and Lohr, 1999) From left: oscillating mirror, Palmer scan, rotating polygon, fiber scanner.

Due to the structure of scanners, elevation data collected at the margin area of the swath shows distinct properties and needs to be removed from the dataset. Usually there are certain overlapping parts between the adjacent strips to ensure those areas are normally sampled.

In the control and processing unit, control and monitor equipment integrate and synchronize components to ensure efficient and error-free functionality of the system. Data storage equipment offers depository media for data collected. 
POS contributes critical auxiliary data to dataset and it consists of two components: an inertial measurement unit (IMU) and a differential global positioning system (DGPS). When topographic LiDAR is in operation, the IMU measures velocity and position adjustments of the aircraft, thus the pitch, roll and yaw of the aircraft can be acquired. The DGPS contains an on-board GPS receiver and a base GPS station on the ground within the vicinity of the operation site, GPS mounted on the aircraft geo-references the sample points and the base GPS station can correct the inaccuracies of on-board GPS to insure high precision of geo-representation of a dataset.

\subsection{Summary of Main Topographic LiDAR Systems}

Table 2.1 lists topographic LiDAR systems from five main manufacturers worldwide. Compared with major technical parameters of topographic LiDAR systems summarized by Baltsavias (1999c), the functionality of current systems has dramatically improved. Scanning angle augments by $20^{\circ}$ to $60^{\circ}$. Pulse rate reaches above $200 \mathrm{kHz}$, almost quadruples their predecessors. Range accuracy increases to around $5 \mathrm{~cm}$ in contrast to more than $10 \mathrm{~cm}$ a couple of years ago. The flying height has also increased, but not as much as other parameters. The current systems can operate on higher altitude, larger area, with a denser sampling rate and shorter time interval. 
Table 2.1 Main manufacturers of topographic LiDAR systems (modified from GIM International)

\begin{tabular}{|c|c|c|c|c|c|}
\hline Manufacturer & Leica & Optech & Riegl & Fugro & TopoSys \\
\hline Type/name of scanner & ALS50-II & $\begin{array}{l}\text { ALTM } \\
\text { Gemini }\end{array}$ & LMS-Q560 & $\begin{array}{l}\text { FLI-MAP } \\
400\end{array}$ & Harrier 56 \\
\hline Dimension \& weight & $\begin{array}{l}37 \times 56 \times 24 \mathrm{~cm}, \\
30 \mathrm{~kg}\end{array}$ & $\begin{array}{l}26 \times 19 \times 57 \mathrm{~cm} \\
23.4 \mathrm{~kg}\end{array}$ & $\begin{array}{l}56 \times 20 \times 22 \mathrm{~cm} \\
20 \mathrm{~kg}\end{array}$ & $\begin{array}{l}50 \times 30 \times 30 \mathrm{~cm} \\
30 \mathrm{~kg},\end{array}$ & $\mathrm{~N} / \mathrm{A},>15 \mathrm{~kg}$ \\
\hline Wavelength & $1,064 \mathrm{~nm}$ & $1,060 \mathrm{~nm}$ & $1,500 \mathrm{~nm}$ & $1,500 \mathrm{~nm}$ & $1,550 \mathrm{~nm}$ \\
\hline Pulse length & $<9$ ns & $7 \mathrm{~ns}$ & $<4 \mathrm{~ns}$ & $4 \mathrm{~ns}$ & $<4$ ns \\
\hline Scanning method & $\begin{array}{l}\text { oscillating } \\
\text { mirror, }\end{array}$ & $\begin{array}{l}\text { oscillating } \\
\text { mirror }\end{array}$ & $\begin{array}{l}\text { rotating } \\
\text { multi-facet } \\
\text { mirror }\end{array}$ & $\begin{array}{l}\text { rotating } \\
\text { mirror }\end{array}$ & $\begin{array}{l}\text { rotating } \\
\text { multi-facet } \\
\text { mirror }\end{array}$ \\
\hline Max. pulse frequency & $150, \mathrm{kHz}$ & $167 \mathrm{kHz}$ & $200 \mathrm{kHz}$ & $250 \mathrm{kHz}$ & $200, \mathrm{Khz}$ \\
\hline Max. scanning angle & $75^{\circ}$ & $50^{\circ}$ & $60^{\circ}$ & $60^{\circ}$ & $45^{\circ}$ or $60^{\circ}$ \\
\hline Max.\#. of echoes/pulse & 4 & 4 & unlimited & 4 & Unlimited \\
\hline Range precision & $<10 \mathrm{~cm}$ & $0.05 \mathrm{~cm}$ & $2 \mathrm{~cm}$ & $2-3 \mathrm{~cm}$ & $5-30 \mathrm{~cm}$ \\
\hline Cameras & $\begin{array}{l}1.3 \mathrm{MP} \text { digital } \\
\text { frame camera }\end{array}$ & $\begin{array}{l}\text { Rollei } 39 \\
\text { Mpixel }\end{array}$ & $\begin{array}{l}\text { IGI } \\
\text { DigiCAM }\end{array}$ & $\begin{array}{l}11 \mathrm{Mpix} \text { still } \\
\text { and video }\end{array}$ & $\begin{array}{l}\text { Applanix } \\
\text { POSAV } 410\end{array}$ \\
\hline Fly height & $200-6,000 \mathrm{~m}$ & $200-4000 \mathrm{~m}$ & $\begin{array}{l}30 \mathrm{~m} / 500 \mathrm{~m} / \\
1,000 \mathrm{~m}\end{array}$ & $50-400 m$ & $\begin{array}{l}30 \mathrm{~m} / 800 \mathrm{~m} \\
/ 1,000 \mathrm{~m}\end{array}$ \\
\hline Max. operation time & $\sim 17 \mathrm{hrs}$ & unlimited & $\sim 8 \mathrm{hrs}$ & $3-6 \mathrm{hrs}$ & $>8 \mathrm{hrs}$ \\
\hline
\end{tabular}

Besides the above advantages, current systems have two properties which were not well established in previous systems: multiple echoed recording and a digital camera. Multiple echoes occur when the LiDAR beam penetrates the surface of the object (e.g., tree canopies) or hits the border portion of objects (e.g., edge of a building). By applying these extra data, classification in the dense urban area can achieve higher accuracies. A digital camera has become a standard component of today's topographic LiDAR systems. Topographic LiDAR 
is a single wavelength detector. Its gray scale images are obscure to visualize by naked eye while on board camera enables real-time views of the scanning surface underneath the flight route by offering images (multiband or visible band) at a fixed time interval. Combining these two kinds of images, true colour 3D imagery can be generated.

\subsection{Basic Ranging Formulas}

In topographic LiDAR ranging, two principles are utilized: pulse ranging (PR) and phase ranging (FR). PR calculates the time interval between emitted LiDAR beam and its echo, FR measure the phase difference between the transmitted and the returned LiDAR beam through continuous wave (CW) LiDAR beam generation. Both principles measure the travel time of the signal, however, different physical effects are utilized (Wehr and Lohr, 1999). Since the pulse LiDAR beam is applied in most of the systems (Wehr and Lohr, 1999, Flood, 2001, Pfeifer and Briese, 2007), this section will mainly describe formulas related to PR.

In the early stages of topographic LiDAR research, rich sets of formulas were examined by some renowned contributors in the community (Baltsavias, 1999b; Wehr and Lohr, 1999). In the following sections, basic formulas are elaborated for the interest of remote sensing. For simplicity, the attitude of aircraft is assumed zero while the flying height and ground speed are assumed as constants.

1. Range 
Range is the distance from LiDAR scanner to ground surface, and is represented by $R, \mathrm{c}$ is the velocity of light, $t$ is traveling time of LiDAR beam captured by counter.

$$
R=\frac{1}{2} c^{*} t
$$

where $t$ is the round trip time and needs to be divided by two.

2. Maximum Range

$$
R_{\max }=\frac{1}{2} c^{*} t_{\max }
$$

The maximum range $\left(R_{\max }\right)$ is limited by the maximum traveling time $\left(t_{\max }\right)$ of LiDAR beam and the time that could be captured by the counter in the scanner.

3. Range Accuracy

$$
\delta \sim \frac{1}{2} c \lambda * \frac{1}{\sqrt{S / N}}
$$

Range accuracy $(\delta)$ is dependent on the wave length $(\lambda)$ of the LiDAR beam and inversely proportional to the square root of signal to noise ratio $(S / N)$. Typical factors contributing to the ratio are the cloud condition in the sky, the power of received signal and radiation property of the terrain surface.

\section{LiDAR Beam Divergence}

Typically the value of instantaneous field of view (IFOV) is applied to describe the divergence of LiDAR beam. IFOV is determined by the wave length of LiDAR $(\lambda)$ and the diameter of the aperture $(D)$ on the ranging unit. 


$$
I F O V=2.44 \frac{\lambda}{D}
$$

The emitted LiDAR beam and its echo share the same aperture, which ensures that the terrain surface covered by the LiDAR beam is always within the receiver's field of view. The value of IFOV is normally between $0.3-3 \mathrm{mrad}$.

\section{LiDAR beam Footprint Diameter}

As shown in Figure 2.2, let $h$ be the flying height of the aircraft and $\alpha$ be the half angle value between emitted LiDAR beam and its echo.

$$
w=2 h^{*} \tan (\alpha)
$$

Since $\alpha$ is relatively a small value, $w$ can be approximately evaluated by the following equation.

$$
w=2 h * I F O V
$$

where $h$ is measured in metre and IFOV in mrad.

6. Scanning Swath

Let $Q$ be the swath width of the topographic LiDAR system, $h$ is the flying height of the aircraft and $\beta$ is the swath angle of the scanner.

$$
Q=2 h * \tan \left(\frac{\beta}{2}\right)
$$

7. Minimum Required Number of Strips

Multiple strips are required if the width of target area is larger than the scanning swath of the topographic LiDAR system. Let $N$ be the minimum number of strips, $L$ 
is the width of ground surface region of interest, $Q$ is the scanning swath and $p$ is the overlapping fraction.

$$
N=\frac{L}{(1-p)^{*} Q}
$$

When $N$ is not an integer, one more strip is needed to complete the mapping.

8. Point Density

Point density refers to the number of sampled points within a unit area (usually one square metre). A higher point density can achieve more accurate rate in mapping elevation data. Let $d$ be the point density, $f$ is the frequency of LiDAR, $l$ is the strip length, $v$ is the velocity of aircraft, $N$ is the number of strips and $A$ is the total area covered.

$$
d=\frac{f^{*}\left(\frac{l}{v}\right)^{*} N}{A}
$$

Two main factors influence the point density: the design of ranging unit, and the flying speed; the lower speed, the higher the point density.

\subsection{Segmentation of Topographic LiDAR Point Clouds}

The topographic LiDAR raw data consist of a combination of terrain, buildings, vegetation, roads and other man-made structures (Charaniya et al., 2004). There have been a large number of works concentrating on data mining from topographic LiDAR data and they can be classified into two categories: filtering and segmentation. In this section both approaches 
are reviewed in detail.

Filtering refers to the elimination of points caused by reflections of LiDAR pulses on vegetation and buildings (Vosselman and Maas, 2001). After filtering, ground and non-ground points are separated. Ground points are widely employed for generating DEM, which is essential for many topographic, hydrographic, agricultural, and construction applications (Fowler, 2001). Above terrain features can be further extracted from non-ground points and building footprint derivation is one of main tasks. Most topographic LiDAR data filtering techniques consist of morphology, slope and surface approaches.

Morphology filters are very popular in optical image processing and were introduced to handle topographic LiDAR data in the 1990s. Kilian et al. (1996) applied the lowest elevation value plus a certain band width threshold (determined by accuracy level of LiDAR scanner) within a moving window to remove building roof and vegetation points. Zhang et al. (2003) introduced a progressive morphology filter, which engaged a series of windows whose size grew in increasing order to generate DEM. Combined with elevation difference thresholds, the algorithm can create smoother DEM without pre-defining the size of windows and with improved efficiency. Arefi and Hahn (2005) designed construction element opening which held feature needs to be removed through morphology filtering, by overlaying opening with rasterized topographic LiDAR image and imposing feature constraints like shape, size 
and orientation, not only DEM, but also buildings and vegetation can also be separated.

Slope-based filters were developed by Vosselmann (2000). It utilizes the slope of line connecting any two points in topographic LiDAR data set as benchmark to determine terrain points. The algorithm assumes that inclinations among ground points are obviously distinct from those between ground and non-ground points, by comparing gradients with pre-defined threshold to detect terrain points. Roggero (2001) constructed a local linear regression model to estimate intercept, gradient and its standard deviation. Then, a curve function of ground points is established based on these parameters to separate terrain points. Buildings and vegetation can be further segregated from non-terrain points by variance difference in their spatial distributions. Sithole (2001) modified Vosselmann (2000)'s algorithm by introducing a threshold variable, its value varies with respect to the steepness of the terrain and is acquired by computing slope map based on gridded minimum height image in which pixel values are the least elevations within the local neighborhood.

In surface-based filter approach, mathematical equation which best describes the curve of terrain is established. Kraus and Pfeifer (1998) designed weight functions to recursively remove non-terrain points above interpolation surface. After each iteration, the points remaining are getting closer to the actual ground elevation until the cycle limit is reached. Schickler and Thorpe (2001) modified Kraus and Pfeifer (1998)'s algorithm by 
incorporating the concept of surface classes to guide the estimation process and additional curvature and slope constraints to control the shape of the estimated surface. With auxiliary mass-points and break line data, the algorithm can create smoother terrain surface model with a reduced noise level. Lee and Younan (2003) added a post processing step to optimize the result acquired from Kraus and Pfeifer (1998)'s algorithm where the terrain points obtained were compared with the original topographic LiDAR data to extract the matched points with identical georeference characteristics, then interpolation was implemented for refinement.

The performances of different filtering algorithms were reviewed by Sithole and Vosselman (2004). They concluded that none of them are capable of handling every kind of data reflecting various terrain types. All above mentioned filtering algorithms suffer from different problems. The choice of moving window size is critical in morphological filters. The ideal way to locate it with respect to the original topographic LiDAR data is still under investigation. In slope filtering, deciding how to locate the optimal slope of a point with its neighboring points which matches real terrain situation is not finalized yet. Surface-based filtering algorithms require long processing time and it is difficult to extract the exact edge points because the scan data are made up of discrete points and edge points are not always included in the scan data (Woo et al., 2002). Another common issue for filtering algorithms is reformatting. As a pre-processing step, irregular distributed 3D points are converted into rasterized images, the nodes of the gridded network have to be constructed by interpolation 
in the original data set, and consequently some of the information will be lost (Roggero, 2001). In recent years, some researchers started to apply new filtering algorithms on point cloud data directly. Shan and Sampath (2005) proposed a two directional labeling approach to generate DEM. Lin and Wu (2006) presented sweep line method to extract off-terrain points.

In segmentation, points are grouped into segments according to some homogeneity criterion (Tovari and Pfeifer, 2005). In most cases, result of segmentation reveals topographic LiDAR data more explicitly than simply terrain points and object points, so filtering can be regarded as special case of segmentation, which results in two groups without regard to what features are involved in point cloud data. Traditional segmentation approaches can be classified into two categories: edge detection and region growing.

Edge detection is performed by searching the discontinuities along the borders of closed terrain features. Fan et al. (1987) employed zero-crossings and local extrema of curvature along a given direction to extract edge points, by grouping these points into different classes, important physical properties are distinguished from range data. Brovelli et al. (2002) applied spline threshold to separate ground points and non-ground points, then edge points are connected, if edges are closed and heights of points within edges are greater than the mean heights of edge points, then they are labeled as terrain features. This algorithm is 
implemented in GRASS (Geographic Resources Analysis Support System). ThuyVu and Tokunaga (2002) engaged wavelets to partition range images (converted from topographic LiDAR raw data), then object edges are detected using scale factor, as a result, features with different sizes and shapes are distinguished.

Region growing, on the other hand, conglomerates the points sharing similar geometrical properties or belonging to the same terrain features. Jiang and Bunke (1994) reported a straight line based method. A small portion of lines are selected by optimal criterion as seed region, neighboring lines are added into the region until no new line segments are detected in the data set. Lee and Schenk (2001) utilized Delaunay triangulation to represent topographic LiDAR raw data and then those triangles are grouped within adjacency area according to plane parameters and the roughness. Finally patches with similar geo-spatial properties are merged to form meaningful terrain features. Gorte (2002) introduced a Triangulated Irregular Network (TIN) approach, the algorithm iteratively merges TIN meshes created from topographic LiDAR raw data into planar segments by calculating similarities among adjacent triangle meshes. After each iteration, planar segments become larger until all the small triangle structures are properly grouped.

Both of aforementioned approaches have some limitations. Edge-based methods run short when a portion of an edge shows a small difference or when regions are homogeneous (Woo 
et al., 2002) or the edges are not closed. Region-growing methods have problems in noisy data and data in which points from different surfaces overlap (Sithole, 2005). Besides, some of the algorithms still suffer from information loss resulting from rasterization. In recent years, novel techniques to segment topographic LiDAR data have been introduced with some of the typical ways including a split and merge approach (Wang and Tseng, 2004), a graph approach (Sithole and Vosselman, 2005), an object approach (Lohmann, 2002) and a scanning line approach (Han et al., 2007).

\subsection{Building Outline Extraction and Regularization}

Building outlines refer to roof boundaries that segregate the building areas from other terrain features like vegetation or the ground. There are two general assumptions about the building outlines: walls of the buildings are perpendicular, roof areas equal to building planar coverage. These assumptions simplify the work and can produce more generalized building models.

Since LiDAR beams are randomly emitted during flight operation, some points may be missing at the building roof boundaries or shadowed by trees nearby the building. Usually building edge points extracted are zigzagged and further refinement called regularization is needed to regularize the shape of the building roof before they can be employed in a geo-database for3 $\mathrm{D}$ building reconstruction. 
In the early stage of 3D building modeling, due to the limitations from both topographic LiDAR technology and immaturity of algorithms, the point cloud data are rasterized before processing. Meaningful building outline extraction and regularization operations cannot be performed on raster data. There some popular remedies including digital cadastral map (Haala and Brenner, 1997), ground plans (Vosselman and Dijkman, 2001), aerial images (Huber et al., 2003) and orhoimages (Kim et al., 2006) to provide regularized building shapes for 3D city modeling.

In recent years, as scanning rate and accuracy level increasè, it becomes possible that $3 \mathrm{D}$ building models can be reconstructed solely from topographic LiDAR raw data without rasterization and other supporting data. After points covering building roofs are detected, edge points have to be separated from non-edge points in order to estimate building roof outline. A traditional convex hull algorithm is the start point of most edge point extraction algorithms and some modifications are applied to make the algorithm work for various shaped buildings. Lee et al. (2007) separated building points by grids and restricted the search space within current and adjacent grids. By joining the edge points within each grid, building edge points are achievable. The algorithm presented in Sampath and Shan (2007) started with corner edge point, and then calculates localized minimum clock-wise angle repeatedly to trace the next edge point until the start point is reached. 
Line simplification, aiming at reducing the number of boundary segments in a polygon, shares some similarities with building outline regularization. There are a number of algorithms dealing with this application in cartography for decades. Douglas and Peuker (1973) proposed a simple algorithm that recursively eliminates intermediate point if its distance to a polygon is less than distance threshold, otherwise it is maintained. The algorithm terminates when all of the points in the polygon are checked. Jenks (1989) introduced neighbourhood algorithm. It considers three points for each iteration, if the distance from middle point to the line connecting the first and the third point is less than distance tolerance, middle point is discarded and point next to the third point is included in a three point group, otherwise the first point is kept and next three points are chosen to continue calculation until all the points are checked.

The algorithms for building outline regularization from topographic LiDAR point clouds go back in the 1990s. They are based on line simplification algorithms and take the properties of the building into consideration. Weidner and Förstner (1995) presented a minimum description length-based approach. Four points are selected as a group of polylines to fit in one of ten regularization models. With consideration of orthogonality of adjacent polylines, models are created by either reorganizing two middle points or removing one of the middle points. Sampath and Shan (2007) developed a hierarchical regularization approach. First long line segments from building outline are extracted and their linear equations are fixed through 
a least squares solution, then long line segments are divided into groups which are perpendicular each other, finally all line segments are determined by applying the slopes of lines as estimation parameters. Jwa et al. (2008) modified Weidner and Förstner (1995)'s algorithm, such that the directionality all the edge segments are labeled according to compass line filter and weighted with scores, and then three points are chosen in one time to fit in one of three hypothetical solutions by the scores of their line segments.

\subsection{Topographic LiDAR for 3D City Modeling}

The 3D city model consists of landmarks, buildings, vegetation, traffic and transportation networks, etc, among which buildings receive most interest from city planners, environmental managers, commercial organizations and the general public.

Traditionally, 3D objects are reconstructed by two properly angled 2D optical images, which are called a stereo image pair. In the geo-science domain, photogrammetry is a classic, accurate and operational approach for 3D data acquisition (Tao, 2005). However manual 3D processing of aerial images is time consuming and requires the expertise of highly qualified persons (Deng et al., 2004). Researchers are applying multi-sensor data or fusion of data from different sources to recreate 3D city models out of consideration of cost, efficiency and accuracy. 
As a newly emerged remote sensing technique, topographic LiDAR mainly focused on generation of DEMs or Digital Terrain Models (DTMs) at early stage thanks to its unique properties presented in previous section. Several researchers including Kraus and, Axelsson (2000) and Baltsavias et al. (2001) and Pfeifer (1997) examined the suitability through different approaches. Modern topographic LiDAR systems are capable of generating much denser sampling rate (more than $200 \mathrm{kHz}$ ), buildings and other man-made features in urban areas are represented by hundreds of points, which makes it possible to model 3D cities using topographic LiDAR point cloud data.

Due to topographic LiDAR's ability to directly geo-referencing 3D features, it is natural to integrate topographic LiDAR data with existing 2D maps for fast, accurate and highly automated acquisition of 3D maps (Elberink et al., 2006). Haala and Brenner (1997) segmented the Digital Surface Model (DSM) from topographic LiDAR data to extract building regions, then building parameters were defined by a least squares adjustment procedure. Based on these predefined references roof elevations were estimated, with available building map data 3D city model were recovered. Vosselman and Dijkman (2001) applied Hough transform algorithm to extract planner faces of buildings from topographic LiDAR point cloud, by the support of building ground plan, building models were recovered.

Besides 2D maps, optical images are another popular remote sensing data type utilized 
together with topographic LiDAR data to achieve better outcome in 3D city modeling. Huber et al. (2003) extracted building shapes and boundaries from aerial images and DSM from topographic LiDAR data, through fusion of two data layers, accurately positioned 3D buildings were remodeled. Rottensteiner et al. (2003) computed normalized difference vegetation index (NDVI) from green and near infrared bands of the geocoded multi-spectral images, which helped to remove vegetation regions from DSM created from topographic LiDAR data. After traditional morphologic filtering processes, building points were preserved for 3D building retrieval. Kim et al. (2006) suggested a new algorithm to produce the true ortho-images from optical images through a co-registration process with topographic LiDAR data of the same coverage. By draping an ortho-image on top of the DSM, a 3D city model can be created.

Some researchers even piloted to integrate multiple data sources for 3D city modeling. Steed et al., (2004) and Vosselman (2002) applied aerial images, topographic LiDAR data and existing $2 \mathrm{D}$ vector maps to create $3 \mathrm{D}$ city models in a purpose to make best use of available data, reduce work load and achieve higher level of automation.

As topographic LiDAR technology evolves, it has become reality that $3 \mathrm{D}$ city models are created solely from topographic LiDAR raw data without any supporting data, researchers concentrate more on this topic in recent years. Rottensteiner and Briese (2002) proposed a 
subtraction approach. First DSM and DTM are produced through interpolation, non-ground points are picked by subtraction of DSM and DTM, and then building points are filtered out by proper threshold, in final step buildings are reconstructed geometrically by fine filtering and modeling procedures. Hofmann (2004) introduced TIN structure to establish 3D buildings. Topographic LiDAR raw data are re-formatted by TIN pattern, each triangle is recorded by spherical coordinates and those coordinates are displayed in a Cartesian coordinate system. Through clustering algorithm, small TIN patterns are grouped into several clusters, where these clusters are further assembled to form the roof and wall of the building according to certain thresholds. This algorithm can recover a building roof in more detail. Tarsha-Kurdi et al. (2006) proposed new approach by utilizing first returns of topographic LiDAR data to extract 3D buildings. Points of first return contain ground points, building roof points and vegetation crown points, ground points are filter out by proper height threshold, rest of the points are rasterized. By integrating elevation information of each point and a filtering technique in an optical image, building points are extracted and applied for 3D building recovery.

From above analysis it is can be clearly observed that topographic LiDAR data has proven to be a rather powerful source for a wide range of 3D GIS object tasks (Schwalbe et al., 2005). According to Hofmann (2004) and Tarsha-Kurdi et al. (2007) two approaches are adapted for 3D city modeling applications by employing topographic LiDAR data. The first approach is 
model driven, a range of basic building models are established in advance, then $3 \mathrm{D}$ building models are recovered by searching the best matching models in the building model library. Haala, and Brenner (1997) and Maas (1999) took this track. But this approach has constraints and is usually limited to simple building models. Complex ground plans may be split into parts, which can be modeled individually (Haala et al., 1998). The second approach is data driven, in contrary to model driven, the buildings are re-established completely depend on the information from topographic LiDAR data. This approach works on arbitrary shaped building roofs, however, it requires data with a higher sampling rate and more complicated algorithms. Since data driven approach is capable of modeling the 3D cities more faithfully, the majority of researchers focus on this track and most of the literature reviewed in this section belong to this domain.

Though topographic LiDAR is gaining increasing importance over photogrammetry, it does not mean that topographic LiDAR will replace the latter completely in 3D city modeling, because both technologies have their strong and weak aspects. As indicated in Table 2.2, topographic LiDAR is superior in terms of data collection, direct 3D coordinates acquisition and vertical accuracy. Photogrammetry is predominant in semantic and break line information and planimetric accuracy. In the future the choice of one technique over another will mainly depend on case requirements. 
Table 2.2 Comparison between topographic LiDAR and photogrammetry (Kim et al. 2006)

\begin{tabular}{|c|c|c|c|}
\hline \multicolumn{2}{|r|}{ Topographic LiDAR } & \multicolumn{2}{|r|}{ Photogrammetry } \\
\hline \multirow{4}{*}{$\overrightarrow{8}$} & $\begin{array}{l}\text { dense information along } \\
\text { homogenous surfaces }\end{array}$ & \multirow{4}{*}{ 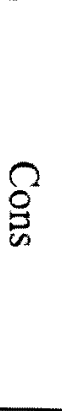 } & $\begin{array}{l}\text { almost no positional information } \\
\text { along homogeneous surfaces }\end{array}$ \\
\hline & day or night data collection & & day time data collection \\
\hline & direct acquisition of 3D coordinates & & $\begin{array}{l}\text { complicated and sometimes } \\
\text { unreliable matching procedures }\end{array}$ \\
\hline & $\begin{array}{l}\text { vertical accuracy is better than } \\
\text { planimetric accuracy }\end{array}$ & & $\begin{array}{l}\text { vertical accuracy is worse than } \\
\text { planimetric accuracy }\end{array}$ \\
\hline \multirow{4}{*}{ 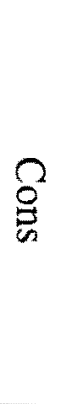 } & no inherent redundancy & \multirow{4}{*}{ ర্ } & high redundancy \\
\hline & $\begin{array}{l}\text { positional; difficult to derive } \\
\text { semantic information }\end{array}$ & & rich in semantic information \\
\hline & $\begin{array}{l}\text { almost no information along break } \\
\text { lines }\end{array}$ & & $\begin{array}{l}\text { dense positional information along } \\
\text { object space break lines }\end{array}$ \\
\hline & $\begin{array}{l}\text { planimetric accuracy is worse than } \\
\text { vertical accuracy }\end{array}$ & & $\begin{array}{l}\text { planimetric accuracy is better than } \\
\text { vertical accuracy }\end{array}$ \\
\hline
\end{tabular}

Based on the observation of properties of both topographic LiDAR and photogrammetry, Rönnholm et al. (2007) proposed a concept of integration of both methods to achieve optimal outcome. Four levels of integration, object-level integration, photogrammetry aided by LiDAR scanning, LiDAR scanning aided by photogrammetry and tightly integrated LiDAR scanning and optical images are elaborated, which provides general guide lines in choosing most appropriate integration to satisfy project criterions.

\subsection{Chapter Summary}

In the first part of this chapter, several topics about topographic LiDAR systems including their composition, basic ranging formulas and properties of current systems are discussed, 
which offers a background for further study. In the second part, different approaches on building detection, building roof outline extraction and regularization, 3D city modeling from topographic LiDAR data are reviewed. The difficulties and challenges remaining in these areas are examined. The objectives and structure of this thesis are presented. 


\section{DESCRIPTION OF TOPOGRAPHIC LIDAR DATA}

In this chapter some important aspects regarding topographic LiDAR data are discussed in detail. They are essential for choice of proper topographic LiDAR data products, algorithm design and data accuracy evaluation.

\subsection{Topographic LiDAR Data Standards}

Topographic LiDAR systems have been developed through increasing demands for high-accuracy and low cost surface elevation data collection. Each manufacturer adopted its own standard regarding topographic LiDAR data collection, data format, accuracy assessment, etc. This pure commercial behavior, on one hand, makes vendors focus on competing to have larger market share while ignoring cooperation in term of facilitating customers to choose desirable topographic LiDAR system wisely, on the other hand, greatly hinder the interoperatability and post processing of topographic LiDAR data.

As topographic LiDAR implicates a wider array of mapping and photogrammetry applications, the industry standards are in high priority for geomatics community in order to regulate the development of this comparatively new technology. The American Society for Photogrammetry and Remote Sensing (ASPRS) has established several guidelines and industry standards which have been widely used by various users. The main works include: 
1. Digital Elevation Model Technologies and Applications: The DEM Users Manual. The second version of this manual was released in 2007 . It covers a wide variety of topics about the DEM including an overview of the topographic LiDAR systems, procedures of creating LiDAR-based DEMs, the advantages and limitations of topographic LiDAR, data processing software and LiDAR-derived DEM accuracy . assessment (Maune, 2008).

2. ASPRS LiDAR Guidelines : Vertical Accuracy Reporting for LiDAR Data This document identifies the vertical accuracy reporting requirement when analyzing elevation data generated using airborne light detection and ranging or laser radar (LiDAR) technology. It consists of three parts: first part lists accuracy requirements (horizontal accuracy and vertical accuracy) when specifying the quality of elevation data; second part deals with Accuracy Assessment and Reporting, starting from designing accuracy tests, selecting and collecting and checkpoints, Deriving Dataset Elevations for Checkpoints, to computing errors and analyzing errors; third part is about how to Calculating and Reporting Vertical Accuracy. (ASPRS LiDAR Guidelines: Vertical Accuracy Reporting for LiDAR Data, 2008).

3. ASPRS LiDAR Guidelines: Horizontal Accuracy Reporting This is the accomplishment of vertical accuracy reporting, where in some cases horizontal accuracy has to meet certain level. In this document, several popular 
minimum horizontal accuracy standard including ASPRS 1990 are listed, as well as their differences and conversion formulas. ASPRS also published Planimetric Accuracy for large scale LiDAR maps. In the last part, common errors and their effect on horizontal accuracy are investigated, correct operation instructions are provided as well (ASPRS LiDAR Guidelines: Horizontal Accuracy Reporting, 2008).

\subsection{Data Format}

Comparing with other imagery widely applied in remote sensing and mapping industries, the information included in the topographic LiDAR data is relatively simple. There are millions ,

of points in one dataset, each point is georeferenced in a geographic coordinate system like Universal Transverse Mercator (UTM) system and assigned with an elevation value. Optionally each point can be associated with intensity value, time tag or colour (red, green and blue) value.

At the initial stage of topographic LiDAR advancement, each manufacture adopted its own data delivery format out of hardware and software properties and requirements from clients. As a result there are many different data formats and they are company-dependent.

Table 3.1 lists major topographic LiDAR formats available in technical reports and academic 
literatures. Except for a few exceptions (index file, project file or DTM file), two data formats, ASCII and binary are widely applied. ASCII is abbreviation for American Standard Code for Information Interchange, it is numerical representation of English alphabet and symbols. .ASC, .DAT, .PTS, .PTX, .RAW, .TXT, .WRL, .XYZ are all generic forms of ASCII format, they are plain text files and can be edited by any text file editors. Binary format takes another path, where all the contents in the file are represented in binary numbers $(0$ and 1$)$, extra information needed to be provided on how to interpret binary number correctly in order to apply various functions on dataset. Formats such as .3DD, .BIN, .LAS, .LDA, .TEW, .TS, .ZFC and .ZFX all belong to this group. 
Table 3.1 Existing common LiDAR data file formats (Samberg, 2007)

\begin{tabular}{|l|l|l|}
\hline Format & Type & \\
\hline .3DD & binary & Riegl \\
\hline .ASC & ASCII & text file \\
\hline .BIN & binary & TerraScan \\
\hline .CMP & propriety & Optech's REALM, comprehensive format \\
\hline .CSD & propriety & Optech's REALM \\
\hline .DAT & ASCII & text file \\
\hline .DVZ & propriety & project file in FUSION/LDV \\
\hline .IXF & propriety & Optech's ILRIS parser \\
\hline .LAS & binary & ASPRS LAS \\
\hline .LDA & binary & FUSION/LDV \\
\hline .LDI & propriety & index file in FUSION/LDV \\
\hline .LDX & propriety & index file in FUSION/LDV \\
\hline .PTC & & TerraScan classification file \\
\hline .PTS & ASCII & Leica Geosystems \\
\hline .PTX & ASCII & Leica Geosystems \\
\hline .QTC & propriety & QT Modeler, ungridded point clouds, no interpolation or \\
& & approximation \\
\hline QTTT & propriety & QT Modeler, surface model, gridded data set \\
\hline RAW & ASCII & raw topographic LiDAR points \\
\hline .TEW & binary & TopEye Mark II \\
\hline .TS & binary & TerraScan \\
\hline .TXT & ASCII & text file \\
\hline .WRL & ASCII & used in 3D range imaging \\
\hline .XLS & worksheet & Microsoft Excel \\
\hline .XML & & DTM file \\
\hline XYZ & ASCII & text file \\
\hline ZFC & binary & Zoller+Fröhlich \\
\hline ZFS & binary & Zoller+Fröhlich \\
\hline
\end{tabular}

Topographic LiDAR data file in ASCII format looks a like a giant table, each tuple delineates a sampled point, specifying its geospatial location ( $\mathrm{x}$ and $\mathrm{y}$ value), elevation value ( $\mathrm{z}$ value) and intensity value, etc. Figure 3.1 shows part of sample data in ASCII format. 


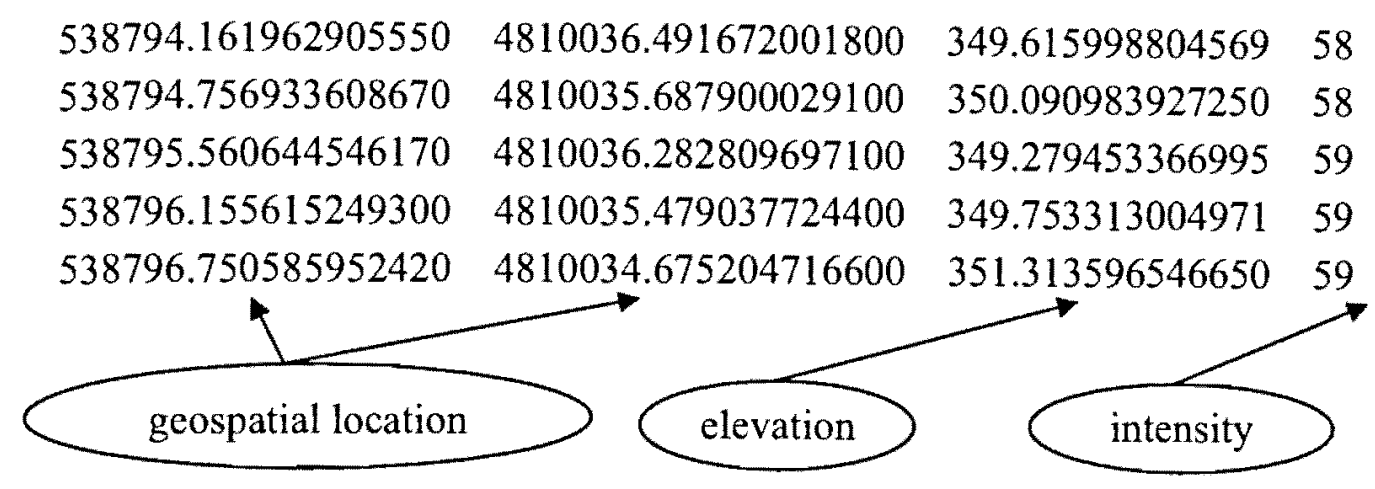

Figure 3.1 Sample topographic LiDAR data in ASCII format

Under this format, topographic LiDAR dataset can be edited on almost any computer without any special software, it also convenient to segment one dataset or combine multiple datasets. This format also widely accepted as input file format by science or engineering computation software like SAS or Matlab.

Figure 3.2 shows the generalized structure of binary file. The header contains general information about the file and information to process the data sections. The relocation table contains records used by the link editor to update pointers in combining binary files. The symbol table holds records used by the link editor to cross reference the addresses of named variables and function between binary files (Inside Mac Media, Inc., 2008). Sections 1 to $\mathrm{n}$ hold the raw data. 


\begin{tabular}{|c|}
\hline Header \\
\hline Relocation Table \\
\hline Symbol Table \\
\hline Section 1 \\
\hline Section 2 \\
\hline$\ldots$ \\
\hline$\ldots$ \\
\hline Section $\mathrm{n}$ \\
\hline
\end{tabular}

Figure 3.2 Generalized structure of binary file (Ung, 1996)

As topographic LiDAR technology advances, it is necessary to have a standard data format that can be integrated by various processing software to simplify the distribution and manipulation of datasets. In 2003, ASPRS published LiDAR Data Exchange Format Standard 1.0, which is referred to as "LAS" format. In 2005, LAS 1.1 was released, while LAS 2.0 is under development. LAS format applies binary format, with file extension ".las". According to ASPRS (LAS format, 2008), the following reasons contribute to the birth of LAS format.

1. Interoperatability. Data cannot be easily taken from one system or process flow to another with proprietary systems.

2. Performance. Processing performance is degraded because the reading and interpretation of ASCII elevation data can be very slow and the file size can be extremely large.

3. Accuracy. All raw data and information specific to the LiDAR data collection is lost. This can inhibit troubleshooting and debugging of problem data sets and limit 
third-party analysis of data integrity

Figure 3.3 illustrates the structure of the LAS format. A complete topographic LiDAR dataset in LAS format 1.1 should carry three parts, public header block, variable length records and point data. In the public header block, general system information like unique file number, type of scanner involved and software information are clarified and data information is also included. Variable length records is developer defined project information, it varies from individual developer. Point data part is the critical part of dataset, $\mathrm{x}, \mathrm{y}$ and $\mathrm{z}$ values, colour and classification of each point are recorded. The full version of LAS 1.1 is accessible through http://www.asprs.org/society/divisions/ppd/standards/asprs_las_format_v11.pdf.

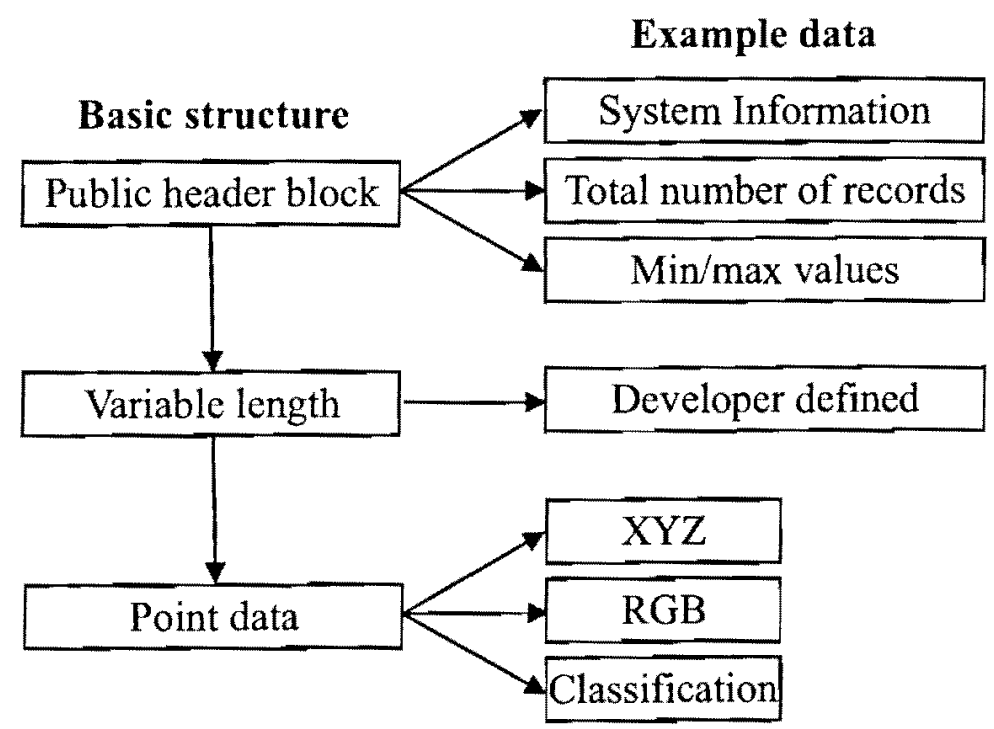

Figure 3.3 LAS format 1.1 (Barber, 2006)

LAS format has be widely accepted both in the USA and internationally so far. Major 
photogrammetric software and topographic LiDAR providers (e.g., Z/I Imaging, Leica Geosystems and Optech), and the US Army Corps of Engineers Topographic Engineering

Center (TEC) have adopted the LAS data format. According to Samberg (2007), some professional organizations including American National Standards Institute (ANSI), The International Organization for Standardization (ISO), ISPRS, The IEEE Committee on Earth Observations Standards Working Group (ISWG) have also started to look over ASPRS LAS. Commercial GIS and image processing software packages including ArcGIS 9.2 Workstation, ENVI 4.3, ERDAS Imagine 9.1, GIS Global Mapper 8.0, Leica Photogrammetry Suite 9.1, QT Modeler 6.0, PCI Geomatica 10.0 all use LAS as the standard input file format. Therefore, LAS format is also used in this study in this study.

\subsection{Data Products}

Collected topographic LiDAR data can be customized or post-processed by a commercial data provider to generate data products with different level of complexity. Flood (2002) defined a series of data products which are commonly accepted by the industry and can be used as guidelines when choosing the best matching topographic LiDAR product from market.

Table 3.2 lists five different levels of products available in general, with costs arranged from the lowest to the highest. Level 1 products contain all the sampled points and have a large data 
Table 3.2Product definitions for topographic LiDAR data (Flood, 2002)

\begin{tabular}{|c|c|c|}
\hline Level & Name & Description \\
\hline 1 & $\begin{array}{l}\text { Basic or } \\
\text { "All } \\
\text { Points" }\end{array}$ & $\begin{array}{l}\text { All of the post-processed topographic LiDAR data properly geo-referenced } \\
\text { but with no additional filtering or analysis. Suitable for those organizations } \\
\text { with in-house data processing tools and capabilities or who work with a } \\
\text { third-party data processing service bureau. Cheapest and fastest product. }\end{array}$ \\
\hline 2 & $\begin{array}{l}\text { Low } \\
\text { Fidelity } \\
\text { or "First } \\
\text { Pass" }\end{array}$ & $\begin{array}{l}\text { Using either proprietary algorithms or third-party software tools, the data } \\
\text { provider will automatically filter the point cloud in to points on the } \\
\text { ground, the "bare earth", and points that are not ground. There is generally } \\
\text { no classification of the non-ground points in to separate features types } \\
\text { (buildings, trees, etc.) and the ground points generally include some } \\
\text { percentage of residual features not extracted by the automated } \\
\text { classification algorithms. Suitable for those organizations with in-house } \\
\text { data processing tools and capabilities or who work with a third-part data } \\
\text { processing service bureau. Common, deliverable. Usually same } \\
\text { cost/schedule as All-Points }\end{array}$ \\
\hline 3 & $\begin{array}{l}\text { High } \\
\text { Fidelity } \\
\text { or } \\
\text { "Cleaned } \\
\text { " }\end{array}$ & $\begin{array}{l}\text { A fully edited data set that has been extensively reviewed by an } \\
\text { experienced data analyst to remove any artifacts created by the automatic } \\
\text { classification routine and provide a " } 99 \% \text { " clean terrain model. The low } \\
\text { fidelity data are analyzed and classified manually, usually with supporting } \\
\text { imagery. Labor-intensive product. Moderate cost but with longer delivery } \\
\text { schedules, especially on larger projects. }\end{array}$ \\
\hline 4 & $\begin{array}{l}\text { Feature } \\
\text { Layers }\end{array}$ & $\begin{array}{l}\text { Further processing using a combination of automated and manual } \\
\text { classification to identify features of interest such as power lines or building } \\
\text { footprints. Generally completed in-house or using a service bureau or } \\
\text { third-party data processor that specializes in the desired application and } \\
\text { has experience or has developed customized tools for the specific type of } \\
\text { feature extraction. Usually more expensive product than high fidelity } \\
\text { terrain model. }\end{array}$ \\
\hline 5 & Fused & $\begin{array}{l}\text { A further refinement of the topographic LiDAR data product achieved by } \\
\text { the fusion of the topographic LiDAR-derived elevation data set with } \\
\text { information from other sensors. This can include digital imagery, } \\
\text { hyperspectral data, thermal imagery, planimetric data or similar data } \\
\text { sources. Generally the most information-rich product with the highest cost. }\end{array}$ \\
\hline
\end{tabular}

volume and the richest information content. Clients must have the ability to extract information desired. In Level 2 products, automatic algorithm is applied to separate ground 
points and non-ground points into layer's structure. The accuracy is limited and cost is same as Level 1 products. Manual involvement is required for Level 3 products to improve the accuracy of Level 2 products, with reference images and trained personnel, high accuracy DEM can be achieved. In Level 4 products, features of interest (trees, buildings, roads, etc) are extracted from non-ground points of Level 3 products, it may employ both automatic and manual classification or tailored software. Level 5 products integrate topographic LiDAR product with information of other sources, it carries abundant information and is the priciest.

\subsection{Error Sources}

Topographic LiDAR system is a complex system, its data file is the fusion of several data sources, errors occur in each sub-system will deteriorate the overall positioning accuracy. Crombaghs et al. (2000) categorized data errors into four components: error per point, error per GPS-observation, error per strip, error per block. Figure 3.4 illustrates the components.

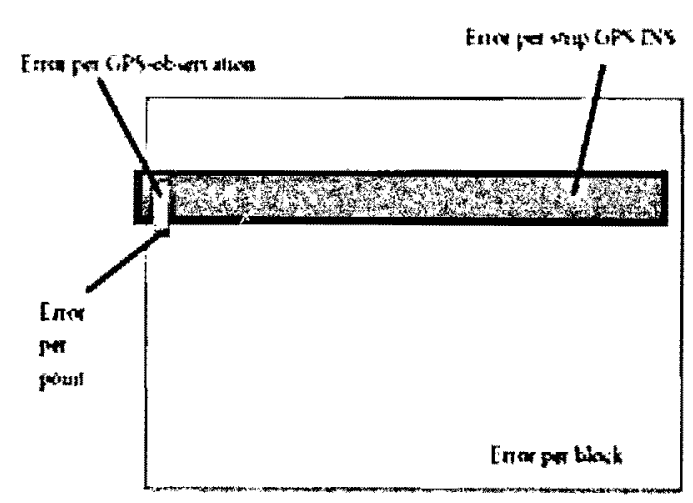

Figure 3.4 Error components of topographic LiDAR data (Crombaghs et al., 2000)

Error per point is introduced by the measuring uncertainty of LiDAR scanner. The cause of error per GPS-observation is similar to error per point, because the GPS time interval is 
larger than the pulse ranging interval, so multiple sampled points within one GPS interval will be influenced. Error per strip happens when integrating both GPS data and inertial navigation system (INS) data, which causes the vertical offsets on every strip during flying operation. Error per block occurs when ground control points are utilized to calibrate the topographic LiDAR dataset, inaccuracies in measuring control points will affect the whole block of dataset which has multiple strips.

Potential error sources contribute to the quality of topographic LiDAR data are examined by Alharthy et al. (2004), Mass (2003), Schenk (2001), Sithole and Vosselman (2003) and Zhang and Liu (2004). They can be grouped into three aspects: systematic errors, random errors and other errors. All types of errors are explained in the following:

Systematic errors can happen in each part of topographic LiDAR system, they are results of deficiencies of equipments or mistakes happened during operation.

1. Ranging Unit and Scanner

These errors include the alignment failure of the emitted LiDAR beam and its echo, the counter's inaccuracy in timing the LiDAR beam's traveling time, scanner mirror vibration and swath angle errors, etc. Total elimination of errors is difficult, but they can be minimized through calibration.

2. GPS errors 
Accurate positioning from the GPS device requires the availability of properly positioned GPS satellite constellation at the time of flying operation, otherwise it might be insufficient to geo-reference sampled data precisely. GPS signal from ground station is applied to correct the distortion of GPS data from satellites, this distance from aircraft in mission to GPS ground stations in another factor, usually the shorter the distance, the better the improvement effect.

\section{INS Errors}

INS consists of IMU and auxiliary computers and it constantly generates the position, orientation and velocity of the aircraft. Initialization errors, misalignment (boresight error), and gyro drifts contribute to systematic errors (Schenk, 2001). The misalignment between the INS system and the scanner is the largest source of systematic error (Morin and Sheimy, 2002) and it is necessary to be addressed in more detail.

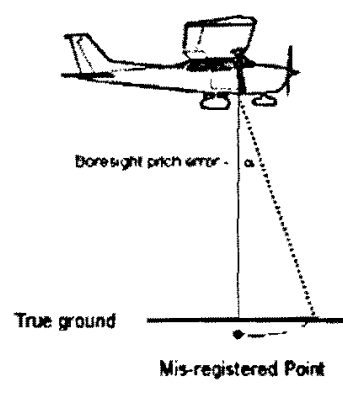

(a)

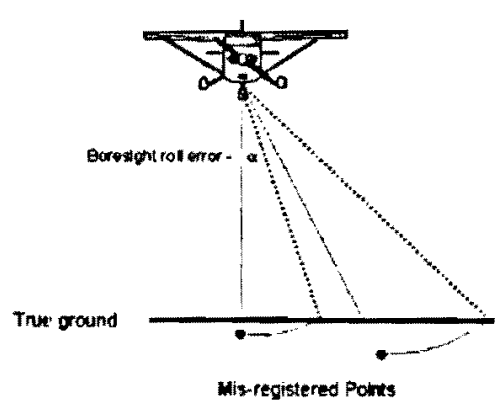

(b)

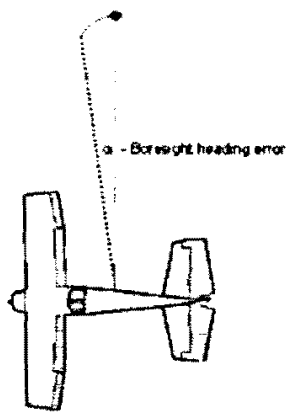

(c)

Figure 3.5 Illustrations of the results of misalignments (ASPRS LiDAR Guidelines:

Horizontal Accuracy Reporting, 2008) 
Pitch error causes inclined recording of nadir (Figure 3.5a), roll error causes wrong range registration (Figure 3.5b), the heading error causes the distortion of each scanning line (Figure 3.5c), deviation scale correlates with angleapositively. Boresight error skews each point within a strip and is removable with reference to the ground control points.

4. Time Bias

Topographic LiDAR systems consist of GPS, INS and ranging components, each component works independently and their sampling rate are distinct, GPS has the slowest rate, ranging unit has the fastest rate. Error occurs when matching three datasets precisely. Schenk (2001) summarized it as time bias, which includes synchronization error and interpolation error. Figure 3.6 demonstrates both types of errors. Synchronization error (Figure 3.6a) occurs when ranging data is available, but the GPS and INS data are absent. Interpolation error (Figure 3.6b) arises when INS data is present, but ranging data is missing. Time bias can be corrected through post processing algorithms.

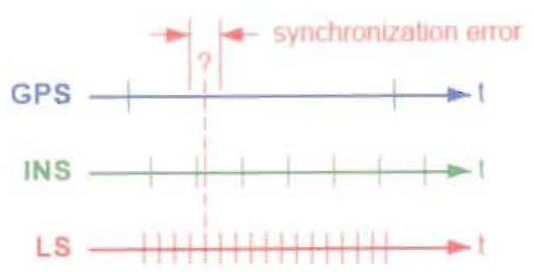

(a)

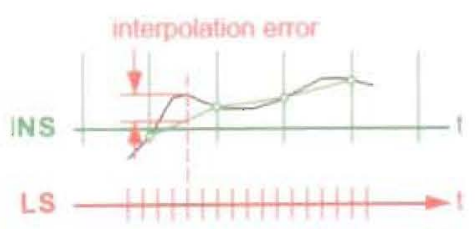

(b)

Figure 3.6 Time bias (Schenk, 2001) 
Pitch error causes inclined recording of nadir (Figure 3.5a), roll error causes wrong range registration (Figure $3.5 \mathrm{~b}$ ), the heading error causes the distortion of each scanning line (Figure 3.5c), deviation scale correlates with angleapositively. Boresight error skews each point within a strip and is removable with reference to the ground control points.

4. Time Bias

Topographic LiDAR systems consist of GPS, INS and ranging components, each component works independently and their sampling rate are distinct, GPS has the slowest rate, ranging unit has the fastest rate. Error occurs when matching three datasets precisely. Schenk (2001) summarized it as time bias, which includes synchronization error and interpolation error. Figure 3.6 demonstrates both types of errors. Synchronization error (Figure 3.6a) occurs when ranging data is available, but the GPS and INS data are absent. Interpolation error (Figure 3.6b) arises when INS data is present, but ranging data is missing. Time bias can be corrected through post processing algorithms.

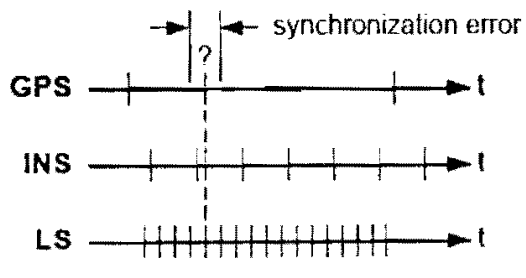

(a)

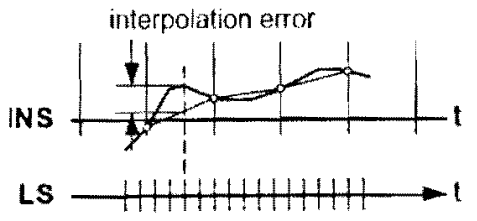

(b)

Figure 3.6 Time bias (Schenk, 2001) 
Random errors arise from topographic LiDAR system designed accuracy limits (vertical accuracy, horizontal accuracy, etc). They cannot be totally eliminated from the system and statistical analysis can minimize these types of errors.

Other errors do not belong to systematic errors or random errors, but they occur and have impact on the data accuracy.

1. Outliers.

Outliers develop when LiDAR beam hit the objects which does belong the topographical features of the earth surface. These objects could include airborne objects like birds or low flying aircraft, or ground objects like pedestrian, cars and animals. Outliers can be easily removed from dataset if their elevations are quite distinct from neighboring features.

2. Atmosphere

As discussed in Section 2.1, emitted LiDAR beam interacts with atmosphere (mainly in troposphere) first before reaching ground. Under unfavorable conditions, air pressure, temperature and humidity may influence the accuracy beyond an acceptable level. These errors can be alleviated by careful mission planning, aircraft maneuver and interpolation in the lab.

3. Human errors

Raw data generation or high level products developing require extensive human 
involvement, which may introduce human errors.

\subsection{Data Ground Processing}

Ground processing can be regarded as generation of a data product. The choice of working procedures and software tools varies from different data providers, but three main objectives (data assembly, data calibration and data customization) must be achieved in this stage. An example from Optech is used to illustrate this process, which will offer snapshot on how data will be manufactured.

Figure 3.7 demonstrates the processing flow of airborne LiDAR terrain mapper (ALTM). First, data stored in hard drives are downloaded to a PC/laptop by the Disk Extraction software. Then range file is decoded into range data and POS data by DashMap software. In stage three, POSPac software is applied to extract both IMU data and air GPS data from POS data, together with basic data processing and adjustment. POSGPS software can create centimeter-level, inertial-aided differential GPS data through combining air GPS data and ground/virtual reference stations (VRS) GPS data. POSProc function from POSPac decodes and reprocesses inertial data using inertial-aided differential GPS information, a Smoother and more accurate (up to 100 times more detailed) Smoothed Best Estimate Trajectory (SBET) is generated. In the last stage, the original point cloud is produced, usually in WGS84 
coordinate system, DashMap can transform XYZ data to local coordinate system decimate and output in desired formats (binary, ASCII, LAS,etc.) and product is ready to be delivered to client.

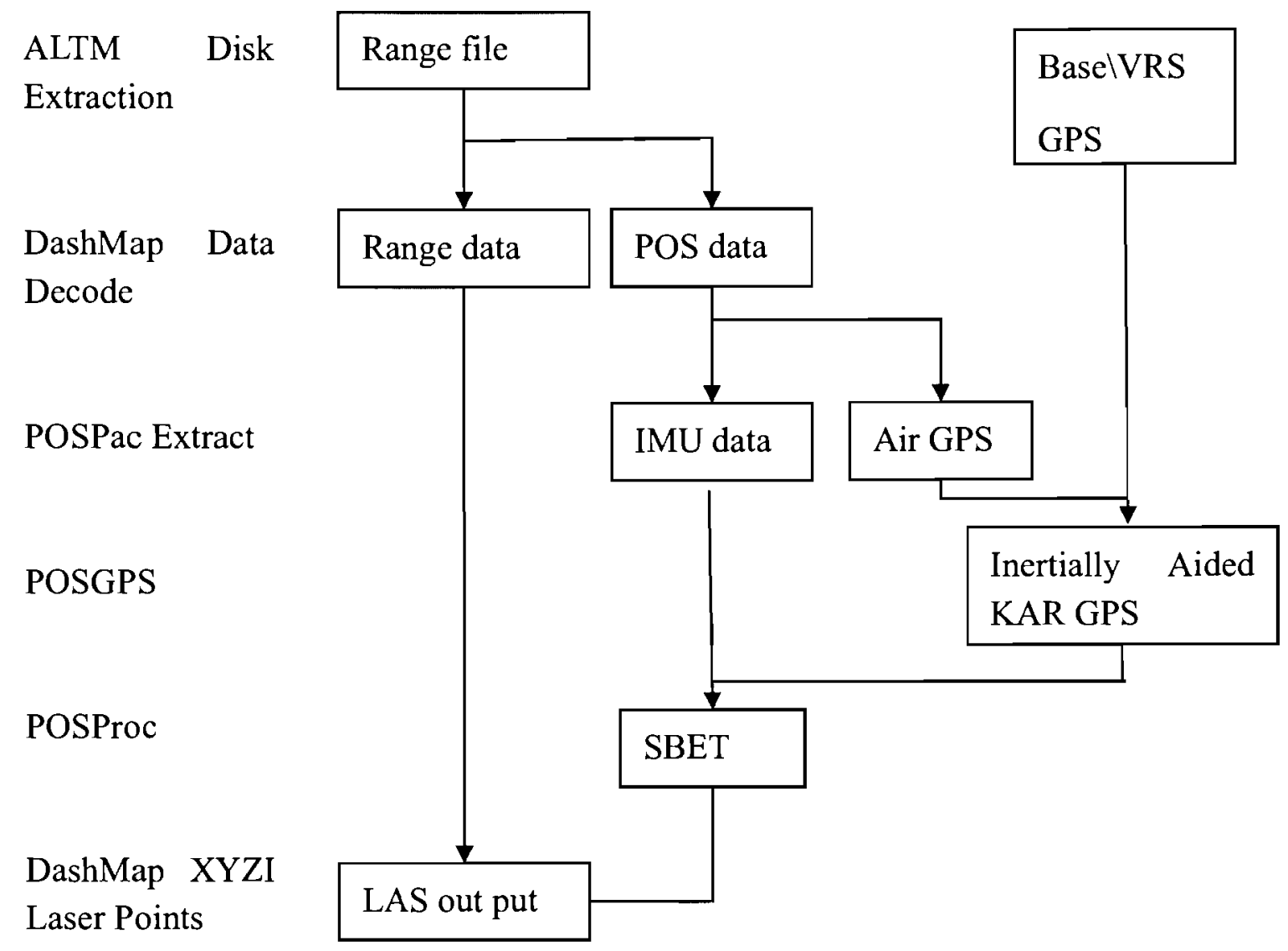

Figure 3.7 ALTM data processing flow (Optech, 2008)

\subsection{Chapter Summary}

Topographic LiDAR developed purely from market demands, which caused confusion and complexity in term of choosing data products. This chapter provides knowledge for topographic LiDAR data standards and formats, which are critical for the design of LiDAR 
data processing algorithms. Main error sources, data ground processing procedures and various data products are also introduced. This background information is beneficial in terms of choosing proper LiDAR products satisfying the requirements of the applications. 


\section{RECONSTRUCTION}

This chapter consists of three sections. First a new topographic LiDAR raw data segmentation algorithm for building coverage detection based on Gaussian Markov Random Field (GMRF) and Markov Chain Monte Carlo (MCMC) is introduced. Then modified convex hull algorithm and a hierarchical regularization algorithm are applied to extract and regularize building roof outlines respectively. Finally, extracted building roof outline points are exported to the ArcGIS to generate 3D building models.

\subsection{Segmentation Algorithm for Building Roof Detection}

This algorithm is applicable for segmenting topographic LiDAR point clouds and can be further divided into two integrated portions. By utilizing GMRF, a mathematical model simulating the distribution of topographic LiDAR point cloud is established and then MCMC is employed to acquire optimal segmentation solution.

\subsubsection{Mathematical Model}

Gaussian distribution, also referred as normal distribution, is one of the basic forms of distribution in statistics and its general formula for the probability density function is expressed as 


$$
y=\frac{1}{\sqrt{2 \pi} \delta} \exp \left\{-\frac{(x-\mu)^{2}}{2 \delta^{2}}\right\}
$$

where $\mu$ is the mean and $\sigma^{2}$ is the variance.

MRFs are stochastic models which describe the spatial relationship among the subset of the data. During the past decades its applications have been extended from mathematical analysis to physics, artificial intelligence and computer vision. Several researchers (Bouman 1995, Perez 1998, Pieczynski and Tebbache 2000, Heesch and Petrou 2007) have applied MRF as a tool for image processing because it provides an efficient and convenient way to model context-dependent features like pixels (Zhang, 2000). In MRF, the correlated relationships among the elements in set $T$ are achieved through neighboring system, which can be defined as $N=\left\{N_{i}, i \in T\right\}$, where $N_{i}$, is neighboring set of element $i(i \in T), i \notin N_{i}$, and $i \in N_{j} \Leftrightarrow j \in N_{i}$. A random field $X$ is MRF within $T$ with respect to defined $N$ if and only if

$$
\begin{aligned}
& p(x)>0, \forall x \in X \\
& p\left(x \mid x_{N_{i}}\right)=p\left(x \mid x_{T-\{i\}}\right)
\end{aligned}
$$

Segmentation is the process of subdividing raw topographic LiDAR data (point cloud) into homogeneous regions, generally as a prelude to further analyses. What should be regarded as homogeneous depends on the context. However, in this study only the elevations are considered. A regression model is specified with a piecewise constant mean function (i.e., a step function) for elevations distributed on an area of interest (AOI) $D$. 
Before going further, concepts of two sets, which are essential during modeling process, need to be set up. The measurements of elevations acquired can be modeled as a random data field $Y$ $=\left\{Y_{1}, \ldots, Y_{n}\right\}$ and a raw topographic LiDAR data on $D$ can be considered as a incomplete random sample of the random field, $y=\left\{y_{1}, \ldots, y_{n}\right\}$. A label indicating the group number to which the corresponding point belongs is represented as a random variable $X_{i}$. For the topographic LiDAR data set with $n$ points and $k$ groups, the collection of all label, $X=\left\{X_{i}, i \in\right.$ $\left.I, X_{i} \in J\right\}$ where $J=\{1, \ldots, k\}, I=\{1, \ldots, n\}$, can be viewed as label field, while $x=\left\{x_{i}, i \in I\right\}$ is one realization of $X$. Figure 4.1 indicates the relationship between label field and data field, for each $y$ value in dataset, there is one and only one $x$ value associating to it.

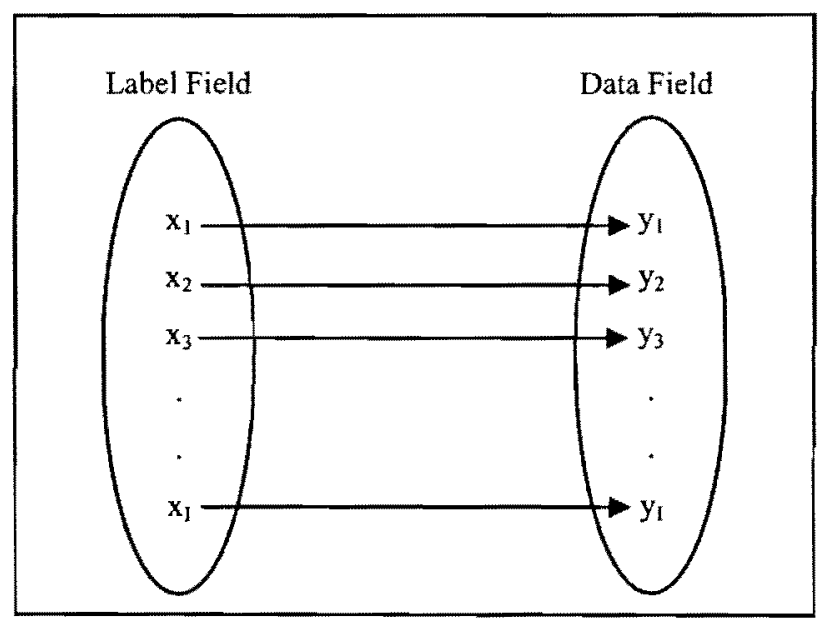

Figure 4.1 Relationship between label field and data field

Given $Y$ (elevations of each point) in a topographic LiDAR raw data set, value of $X$, needs to be optimized such that the all points with similar elevation values are grouped together through finite cycle of computations. The parameter vector $s$ is defined, and $s=\{X, \theta\}$, where $\theta$ is a parameter vector to be estimated, the optimum is achieved by maximizing $p(s \mid Y)$. Using 
Bayes' Theorem, the following equation is obtained:

$$
p(s \mid Y)=p(X, \theta \mid Y) \propto p(X, \theta) p(Y \mid X, \theta)
$$

Assuming $X$ and $\theta$ are statistically independent, therefore

$$
p(X, \theta)=p(X) p(\theta)
$$

Combining Eqs. 4.4 and 4.5, then

$$
p(s \mid Y) \propto p(X) p(Y \mid X, \theta) p(\theta)
$$

Through Eq. 4.6 the maximum of $p(s \mid Y)$ can be calculated by multiplying $p(X), p(Y \mid X, \theta)$ and $p(\theta)$, once the values of above three parts are optimized, $p(X, \theta \mid Y)$ can be obtained. Set $X$, which holds the labels for each point is fixed, thus the whole dataset is segmented according to the labels attached to each point. In the following context, equations to calculate the $p(X), p(Y)$ $X, \theta)$ and $p(\theta)$ will be elaborated.

In a topographic LiDAR raw data set, the distances among points vary in contrast to the regularized distance among pixels in optical images, which introduces extra steps in processing topographic LiDAR data. For label field $X$, it is assumed that the distribution of $x_{i}$ satisfies MRF( i.e., for each independent point of the data set, its elevation is dependent of the elevations of its neighboring points). Since points are randomly scattered, reasonable radium $r$ is designated such that each point has at least one neighboring point and $r$ is determined by the 
intensity of data. As shown in Figure 4.2, the elevation of Point 1 is closely related to the elevations of Points 2, 3, 4 and 5 within square window delineating neighboring zone.

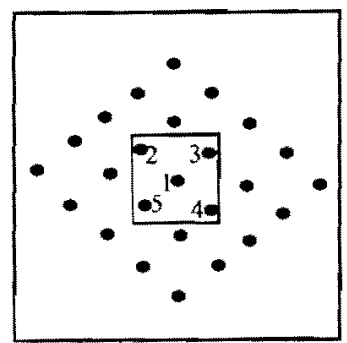

Figure 4.2 MRF property of topographic LiDAR data

$P\left(x_{i}\right)$ is expressed by:

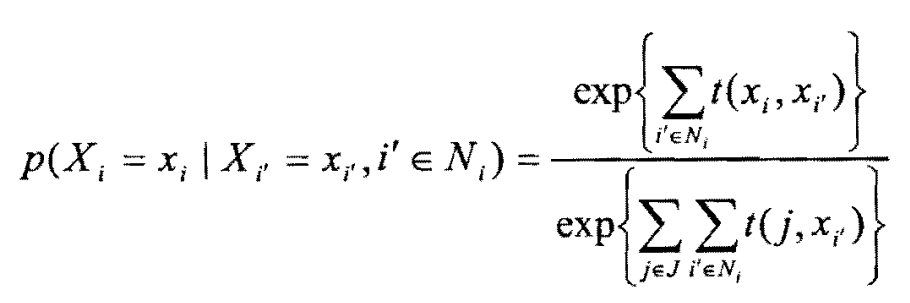

where $t(x, y)=\left\{\begin{array}{ll}1, & \text { if } x=y \\ 0, & \text { otherwise }\end{array}, N_{i}=\left\{i^{\prime}, d\left(i, i^{\prime}\right) \leq r\right\}, d\left(i, i^{\prime}\right)\right.$ is the Euclidian distance between points $i$ and $i^{\prime}, \exp \left\{\sum t\left(x_{i}, x_{i^{\prime}}\right)\right\}$ is the potential power function of $x_{i}$.

By multiplication of $p\left(x_{i}\right), p(X)$ can be obtained by:

$$
p(X)=\prod_{i \in I} p\left(x_{i}\right)
$$

Topographic LiDAR raw data sets can also be viewed as the integration of multiple subsets of points with different $\mathrm{z}$ values, points share same label field within each subset. It is rational to assume that these points satisfy Gaussian distribution with proper mean and variance. For each 
given $x_{i}=j$ the distribution of subset of $y$ sharing the same label $x_{i}$ satisfy Gaussian distribution with mean $\mu_{j}$ and variance $\sigma_{j}^{2}$, we define $\theta_{j}=\left\{\mu_{j}, \sigma_{j}^{2}\right\}$. Thus the probability density function $p\left(y_{i} \mid x_{i}, \theta_{j}\right)$ with reference to Eq. 4.1 can be defined as:

$$
p\left(Y_{i}=y_{i} \mid \theta_{j}=\left(\mu_{j}, \sigma_{j}^{2}\right), X_{i}=j\right)=\frac{1}{\sqrt{2 \pi} \sigma_{j}} \exp \left\{-\frac{\left(y_{i}-\mu_{j}\right)^{2}}{2 \sigma_{j}^{2}}\right\}
$$

Through Eq. $4.9 p(Y \mid X, \theta)$ accomplished as:

$$
p(Y \mid \theta, X)=\prod_{j \in J} \prod_{i \in S_{j}} \frac{1}{\sqrt{2 \pi} \sigma_{j}} \exp \left\{-\frac{\left(y_{i}-\mu_{j}\right)^{2}}{2 \sigma_{j}^{2}}\right\}
$$

For the parameter the vector $\theta_{j}=\left\{\mu_{j}, \sigma_{j}^{2}\right\}, \mu_{j}$ and $\sigma_{j}^{2}$ are assumed to be independent, the joint distribution probability $p\left(\theta_{j}\right)$ can be rewritten as:

$$
p\left(\theta_{j}\right)=p\left(\mu_{j}, \sigma_{j}^{2}\right)=p\left(\mu_{j}\right) p\left(\sigma_{j}^{2}\right)
$$

Assume that mean $\mu_{j}$ follows uniform distribution between minimum and maximum $\mathrm{z}$ values, $\mathrm{i}$. e., $\mu_{j} \sim \mathrm{U}\left(h_{\min }, h_{\max }\right)$, variance $\sigma_{j}^{2}$ follows normal distribution, i. e., $\sigma_{j}^{2} \sim \mathrm{N}\left(0, \tau^{2}\right)$, where $h_{\text {min }}$, $h_{\max }$ and $\tau^{2}$ are constants. For each $\theta_{i}$,

$$
p\left(\theta_{j}\right)=p\left(\mu_{j}\right) p\left(\sigma_{j}^{2}\right)=\left(\frac{1}{h_{\max }-h_{\min }}\right) \frac{1}{\sqrt{2 \pi \tau}} \exp \left\{-\frac{\sigma_{j}^{2}}{2 \tau^{2}}\right\}
$$

Through Eq. 4.12, $p(\theta)$ can be expressed as 


$$
p(\theta)=\prod_{j \in J} p\left(\theta_{j}\right)=\left(\frac{1}{h_{\max }-h_{\min }}\right)^{k}\left(\frac{1}{\sqrt{2 \pi \tau}}\right)^{k} \prod_{j \in J} \exp \left\{-\frac{\sigma_{j}^{2}}{2 \tau^{2}}\right\}
$$

Through the above inference $p(X), p(Y \mid X, \theta)$ and $p(\theta)$ are mathematically formulated by Eqs. $4.8,4.10$ and 4.13 respectively, by Eq. $4.6 p(s \mid Y)$ is solvable by multiplication. Thus the complete GMRF model can be obtained:

$$
\begin{aligned}
& p(s \mid Y) \propto \\
& \prod_{j \in X} \prod_{i \in S, S} \frac{1}{\sqrt{2 \pi} \sigma_{j}} \exp \left\{-\frac{\left(y_{i}-\mu_{j}\right)^{2}}{2 \sigma_{j}^{2}}\right\}\left(\frac{1}{h_{\max }-h_{\min }}\right)^{k}\left(\frac{1}{\sqrt{2 \pi} \tau}\right)^{k} \prod_{j \in J} \exp \left\{-\frac{\sigma_{j}^{2}}{2 \tau^{2}}\right\} \exp \left\{\sum_{\left(i, i^{\prime} \in C_{2}\right)} t\left(x_{i}, x_{i^{\prime}}\right)\right\}
\end{aligned}
$$

where $h_{\max }, h_{\min }, \tau^{2}$ and $k$ need to be fed in manually according the properties of the topographic LiDAR data set.

Figure 4.3 depicts the parameters and their relationships with different parts in Eq. 4.14. $h_{\max }$ and $h_{\min }$ are applied to determine the distribution of mean $(\mu)$ of each group, $\tau^{2}$ controls the jumping step of variance $\left(\sigma^{2}\right)$ for each group, $k$ confines the scope of $X$. With a proper combination of these parameters, optimum values of $X$ could be reached. Thus the meaningful segmentation of topographic LiDAR raw data can be achieved. 


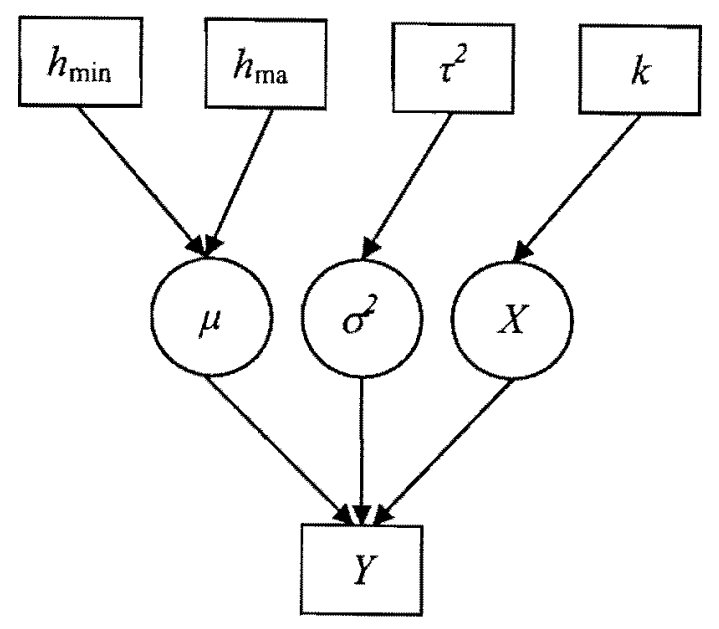

Figure 4.3 GMRF parameters and their interrelationships

\subsubsection{MCMC for Segmentation}

MCMC is essentially Monte Carlo integration using Markov Chains and it mainly focuses on simulation, estimation and optimization and it consists of set of sampling algorithms as long as the distributions satisfy Markov chain properties. In Section 4.1.1 the mathematical model for segmenting topographic LiDAR point clouds set is established by Eq. 4.14. In order to solve this equation, a dependent sampler is required to capture the optimum values of $X$. In this study the MCMC algorithm is chosen based on the following considerations:

1. It is mature and stable theory and has been widely applied in statistical inference.

2. Its main applications involves in Bayesian inference, namely from prior distribution or likelihood established in joint distribution to predict posterior distribution, as described in Eq.4.4.

3. It works better on complicated high-dimensional distributions. A topographic LiDAR 
raw data set has millions of points and it is very difficult simulate its distribution under other type of sampling method like independent sampling.

A standard MCMC algorithm called Metropolis-Hastings algorithm is employed to calculate the optimal value of $X$. Figure 4.4 shows the flowchart of this algorithm and it contains the following six steps: 
Initialize iteration counter $u=1$

Initialize parameter vector $\mathrm{s}^{0}=\left\{\boldsymbol{\mu}^{0},\left(\sigma^{2}\right)^{0}, \boldsymbol{x}^{0}\right\}=\left\{\mu_{1}^{0}, \ldots, \mu_{k \text {, }}^{0}\right.$ $\left.\left(\sigma^{2}\right)^{0}{ }_{1}, \ldots,\left(\sigma^{2}\right)^{0}, x^{0}, \ldots, x_{n}^{0}\right\}=\left\{s_{1}^{0}, \ldots, s_{2 \mathrm{k}+\mathrm{n}}^{0}\right\}$

Initialize component counter $v=1$

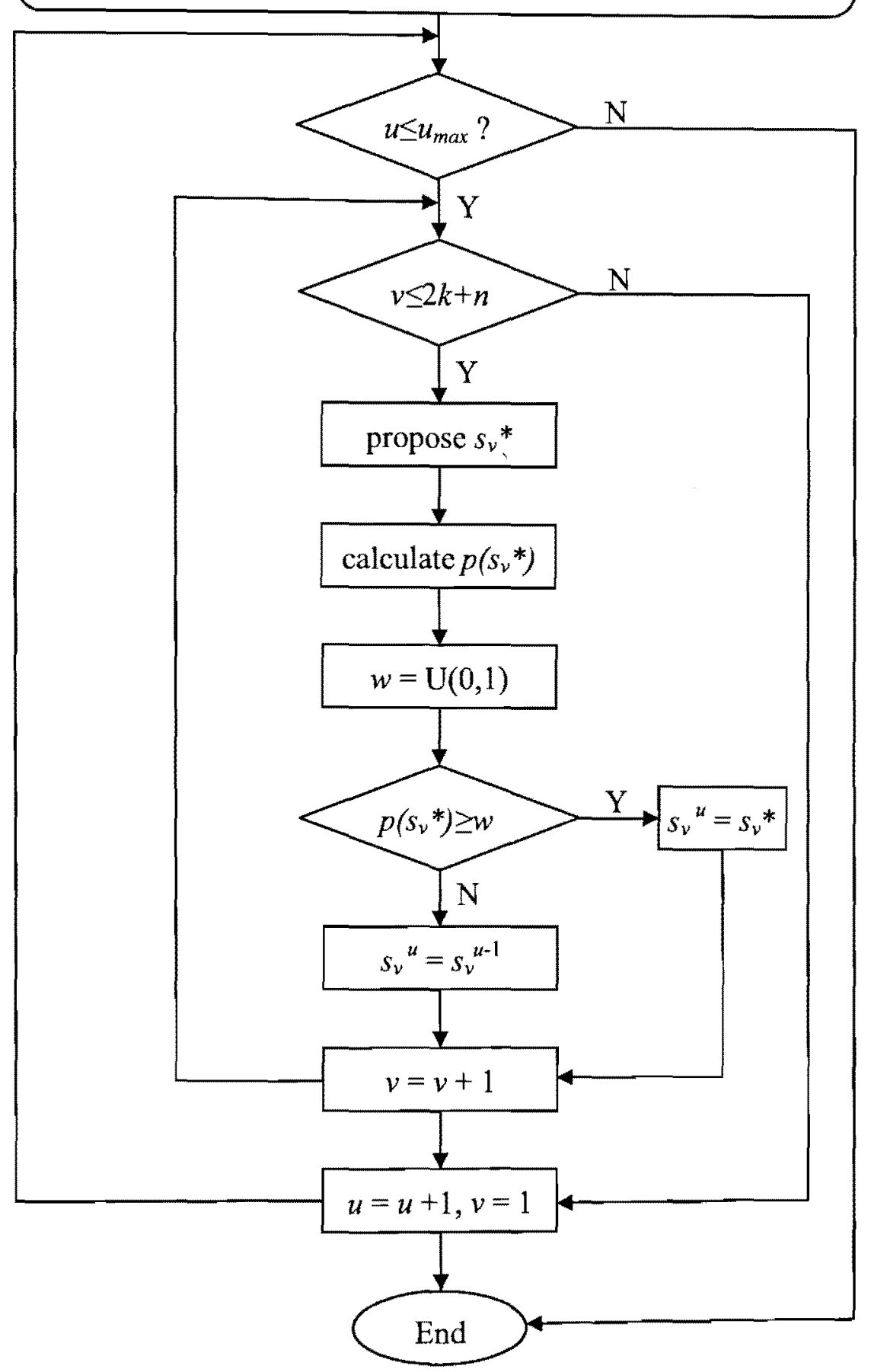

Figure 4.4 Flowchart illustrating Metropolis-Hastings algorithm 
1. Initialize the iteration counter $u=1$ and set the initial value of the chain $s^{0}=\left\{s_{1}{ }^{0}, \ldots\right.$, $\left.s_{m}{ }^{0}\right\}$, where $\mu_{j}^{0}, j \in J$, are drawn from $\mathrm{U}\left(h_{\min }, h_{\max }\right),\left(\sigma_{j}^{2}\right)^{0}, j \in J$, are drawn from $\mathrm{N}(0$, $\left.\tau^{2}\right)$, and $x_{i}^{0}, i \in I$, are uniformly drawn from $\{1, \ldots, \mathrm{k}\}$ and satisfy $S_{j}=\left\{i, x_{i}^{0}=j\right\}, \forall j \in$ $J$.

2. Initialize the component counter $v=1$.

3. Move the $v$ th component $s_{\nu}$ of $s$ to a new value $s_{\nu}{ }^{*}$ generated from the density $q_{s}\left(s_{\nu}{ }^{(u-1)}\right.$, $\left.s_{\nu}{ }^{*}\right)$, where $\mu_{\nu}{ }^{*} \sim \mathrm{N}\left(\mu_{\nu}{ }^{(u-1)}, \varepsilon_{1}\right), \sigma^{2}{ }_{\nu}{ }^{*} \sim \mathrm{N}\left({\sigma^{2}}^{2}{ }^{(u-1)}, \varepsilon_{2}\right)$ and $\mathrm{N}\left(x_{v}{ }^{(u-1)}, \varepsilon_{3}\right), \varepsilon_{1}, \varepsilon_{2}, \varepsilon_{3}$ are constants.

4. Calculate the acceptance probability of the move, $\alpha\left(s_{v}{ }^{(j-1)}, s_{v}{ }^{*}\right)=\min \left\{1, \frac{p\left(s_{v}{ }^{*} \mid Y\right) q\left(s_{v}{ }^{*}, s_{v}{ }^{(j-1)}\right)}{p\left(s_{v}{ }^{(j-1)} \mid Y\right) q\left(s_{v}{ }^{(j-1)}, s_{v}{ }^{*}\right)}\right\}$. Let $w$ be drawn from U $(0,1)$. If $\alpha \geq$ $w$, the move is accepted, then $s_{v}{ }^{(j)}=s_{\nu}{ }^{*}$. If $\alpha<w$, the move is rejected, then $s_{v}{ }^{(j)}=s_{v}{ }^{(j-1)}$.

5. Change the counter from $v$ to $v+1$ and return to step 3 until $v=m$. When $v=m$, go to step 6.

6. Change the counter from $u$ to $u+1$ and return to step 2 until convergence is reached.

\subsection{Building Outline Extraction and Regularization Algorithms}

\subsubsection{Building Outline Extraction Algorithm}

After the segmentation process introduced in Section 4.1 along with a possible filtering 
procedure, building roof points with similar elevation values can be successfully classified. In order to reconstruct the shape of the building roof, building roof edge points need to be further separated from non-edge points. Building roof point determination is a crucial and difficult step in the building reconstruction task (Rottensteiner and Briese, 2002). In this study, the modified convex hull (MCH) algorithm introduced by Sampath and Shan (2007) is adapted to delineate building roof points.

Given a set of points, convex hull is the smallest convex polygon containing all the points, it also can be visualize by wrapping rubber band around the boundary points. Figure 4.5 displays the convex hull of a set of points, it is a closed convex polygon formed by the border points.

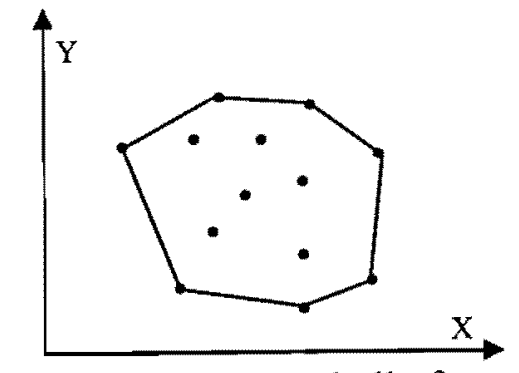

Figure 4.5 Convex hull of a point set

There are different algorithms available to compute convex hull of a set of points like Graham's Scan, Jarvis' March or Quick-Hull. Sampath and Shan (2007) proposed the least clockwise angle algorithm to obtain convex hull. This algorithm is further modified to calculate building roof edge points. 
Figure 4.6 illustrates how this algorithm works. Starting at leftmost point $P$, edges are created with rest of the points within the set. After sorting edges according to their clockwise angles, the edge with the least clockwise angle is chosen to be the boundary edge. Then starting with the other end point of selected edge, the above step is repeated until start point $\mathrm{P}$ is reached. The generated set of edges is the convex hull of the point set.

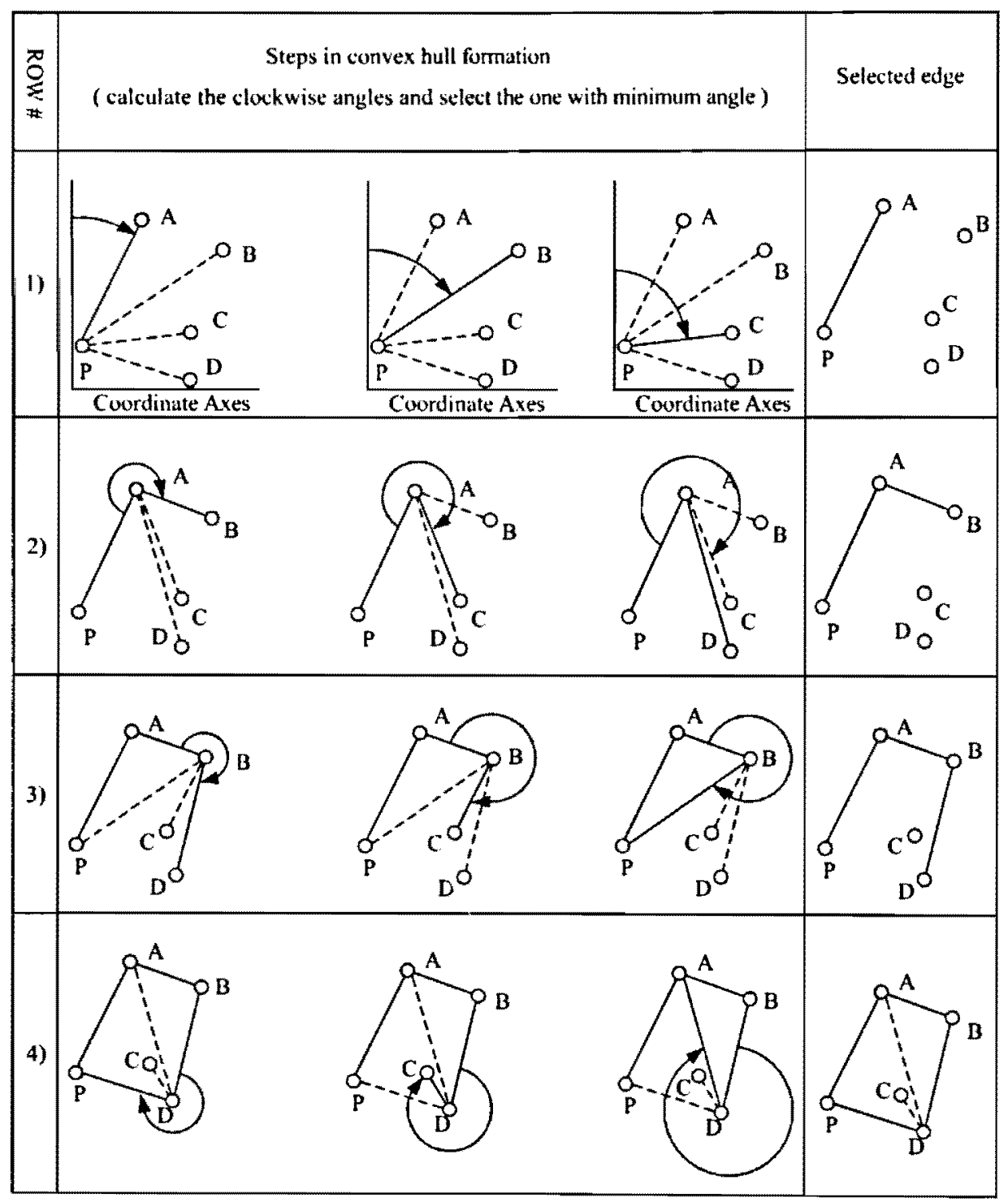

Figure 4.6 Convex hull computation procedure (Sampath and Shan, 2007) 
No matter what shapes the point sets form, the convex hull algorithm computes the convex polygon, which is not always the case in delineating building edge points. Figure 4.7 shows some of commonly seen concave shaped building roofs and their outlines cannot be accurately recovered by the convex hull algorithm.

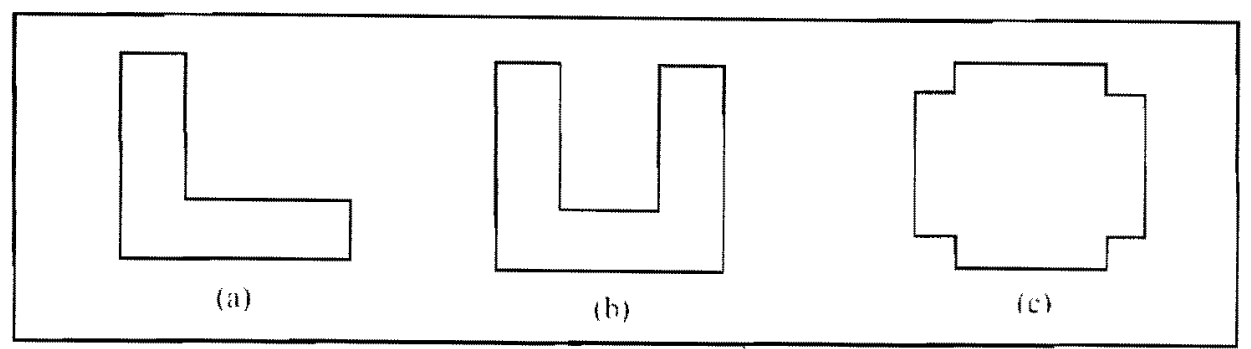

Figure 4.7 Regular concave shaped buildings

In order to trace concave the shaped building roof outline points, $\mathrm{MCH}$ is proposed, which can be regarded as a localized convex hull method. The algorithm creates a moving window for each edge point and correct edge point is traced out within the moving window. After starting point is reached, all edge points are selected successfully regardless of the shape of the building. Figure 4.8 demonstrates the tracing process. At the beginning, a corner edge point is picked, within its neighboring window next edge point is chosen. Starting from the newly selected edge point, the same steps are repeated until the original edge point is encountered. The fourth row in Figure 4.8 shows the distinct results of two algorithms where the building outline recovered from convex hull algorithm is far coarser than the outline from $\mathrm{MCH}$ algorithm and cannot be applied as the base building shape in 3D city modeling. The radius of the moving window should be adjusted according the point density. In general, it is 
slightly larger than twice of the point spacing in the along and across scan directions.

\begin{tabular}{|c|c|c|}
\hline 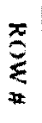 & $\begin{array}{l}\text { Steps in boundary tracing } \\
\text { ( calculate the clockwise angles for points within the neightorhood } \\
\text { of the circle and select the one with minimum angle) }\end{array}$ & Selected edge \\
\hline 1 & 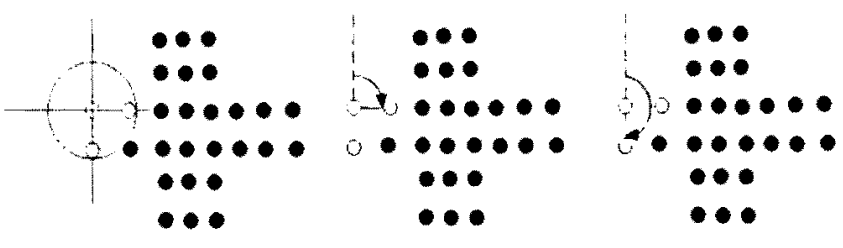 & $\begin{array}{l}\bullet \bullet \\
\bullet \bullet \bullet \\
0 \bullet \bullet \bullet \bullet \bullet \\
\bullet \bullet \bullet \bullet \bullet \bullet \\
\bullet \bullet \\
\bullet \bullet\end{array}$ \\
\hline 2 & 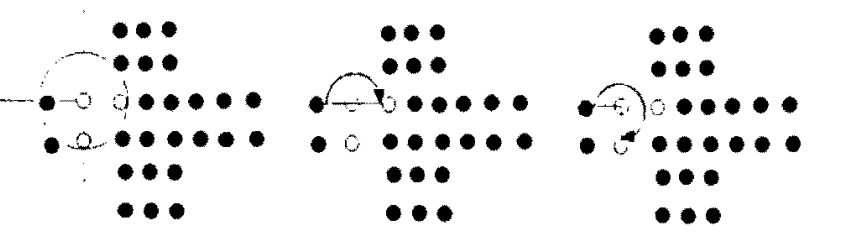 & $\begin{array}{l}\bullet \bullet \bullet \\
\bullet \bullet \bullet \\
0-0 \bullet \bullet \bullet \bullet \\
\bullet \bullet \bullet \bullet \bullet \bullet \\
\bullet \bullet \bullet \\
\bullet \bullet \bullet\end{array}$ \\
\hline 3 & 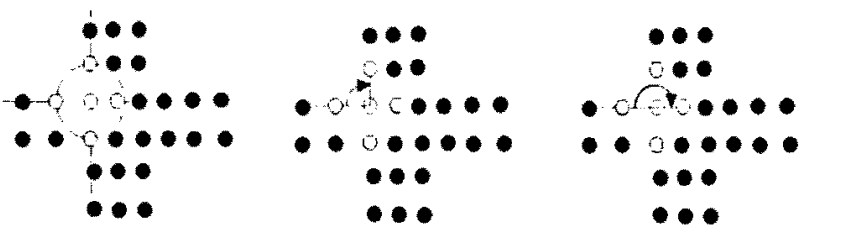 & $\begin{array}{c}\bullet \bullet \bullet \\
0 \bullet \bullet \\
0.0 \quad 0 \bullet \bullet \bullet \bullet \\
\bullet \bullet \bullet \bullet \bullet \bullet \\
\bullet \bullet \bullet\end{array}$ \\
\hline 4 & 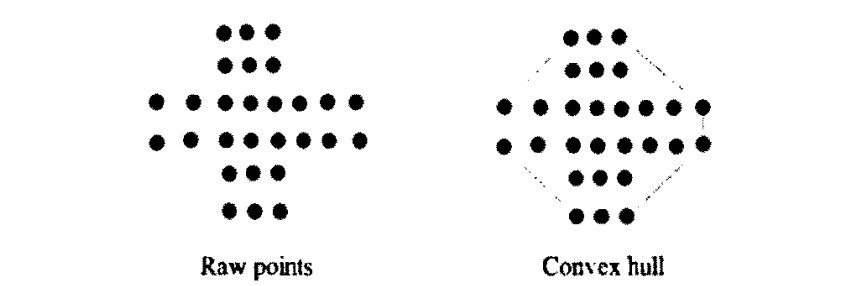 & 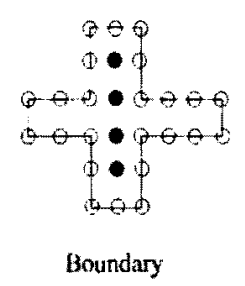 \\
\hline
\end{tabular}

Figure 4.8 Modified convex hull algorithm (Sampath and Shan, 2007)

\subsubsection{Building Outline Regularization Algorithm}

The first step of the algorithm is to locate the line segments framing the shape of building.

According to Sampath and Shan (2007), this is done by sequentially following each building edge point and looking for positions where the slopes of two consecutive edges are 
significantly different. The points on subsequent edges with similar slopes are gathered in one line segment. At the end of this step, the building roof outline points are subdivided into a number of sets of points, the number of sets equals to the number of the building outline segments.

Next step is building roof outline reconstruction. In this study, it is assumed that building edges are straight, so they can be expressed by Eq. $y=a x+b$. The extracted points corresponding to the building outline can be used to estimate the lines. Figure 4.9 demonstrates the process. Given the edge point set $\{A, B, C, \ldots, H\}$, let $d$ be the distance from a point to the line, the line is regressed by Minimum Mean Square Estimate (MMSE), that minimizes the sum of distance, $d_{1}+d_{2}+d_{3}+\ldots+d_{8}$.

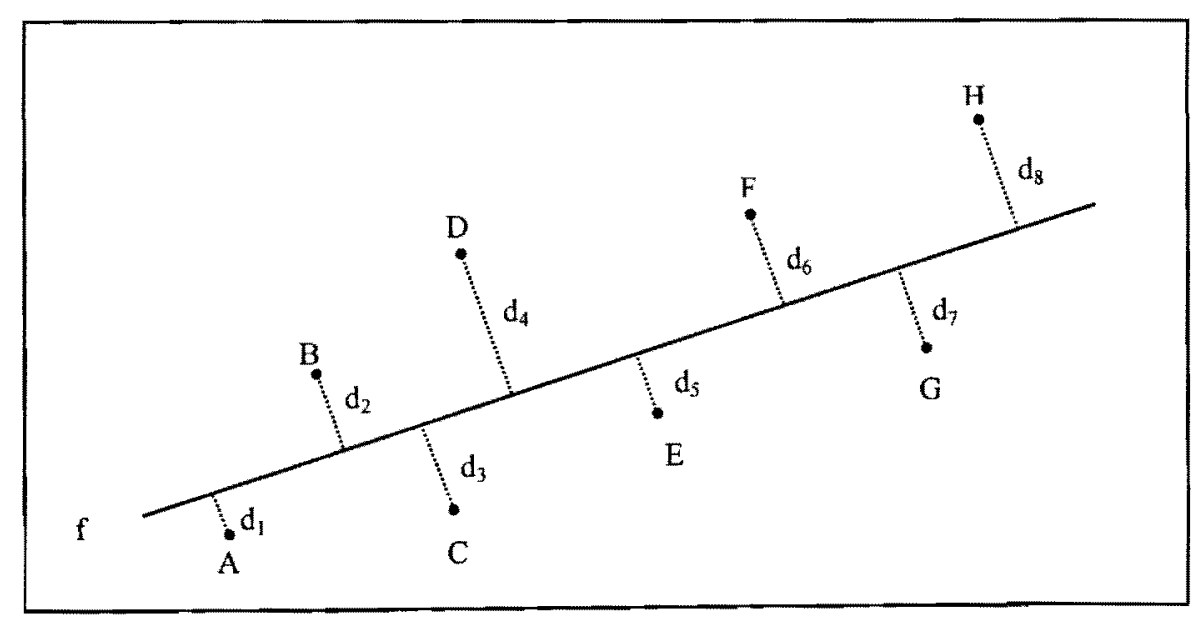

Figure 4.9 Line replacement of multiple points

In the final step, the corner points of a building outline are computed by solving the linear 
equation pairs of adjacent edges. By connecting these points with straight lines, the building roof can be retrieved and are ready to be exported to a geo-database.

\subsection{D Building Generation}

Several software packages are commercially available in the market to produce $3 \mathrm{D}$ city models including Fusion or LP360, ArcGIS is employed in this study.

Figure 4.10 exhibits the key steps in 3D building generation. First, points are converted to a line coverage. A coverage is an intermediate file required by the following function. A polygon shapefile is created based on line coverage. Spatial references of shapefiles are defined, so they can be overlaid on other spatial data. A building polygon shapefile accepts various functions offered by software and can be exported to other geo-databases. During the last step, 3D buildings are generated in ArcScene-3D simulation modular in ArcGIS. 


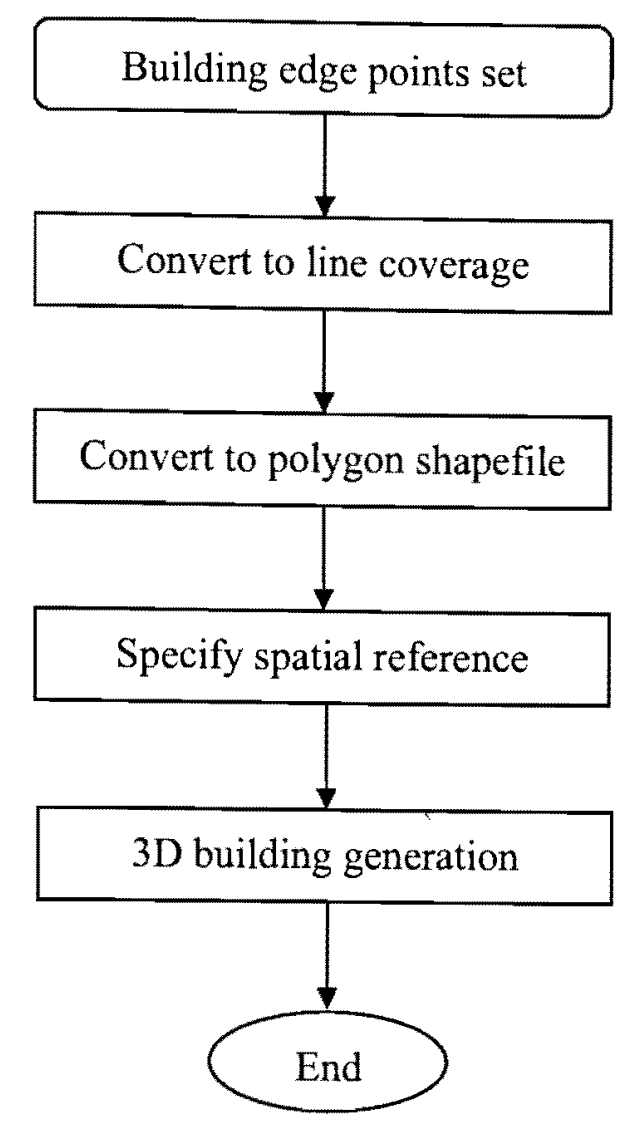

Figure 4.10 Process of 3D building generation in ArcGIS

\subsection{Chapter Summary}

In this chapter, the proposed methodology for $3 \mathrm{D}$ building reconstruction from topographic LiDAR point clouds is described. The complete workflow comprises of three critical parts: building detection, building outline extraction and regularization, 3D building model generation. In the first part, a new segmentation algorithm utilizing GMRF and MCMC is presented. Compared with other algorithms, the advantage of this algorithm is that it works on topographic LiDAR data directly without other supporting data. In the second part, MCH 
and MMSE are applied to obtain the final edge point set that symbolizes planar shape of the building. In the last step, the point set is exported to ArcGIS to generate 3D building models. 


\section{RESULTS AND DISCUSSIONS}

\subsection{Study Area and Data}

The study area is a part of the University of Waterloo (UW) campus, covered by a single strip topographic LiDAR point clouds. Figure 5.1exhibits the image and its legend. Different colours are utilized to symbolize the elevation of each point. The numbers are the absolute elevations in metres.

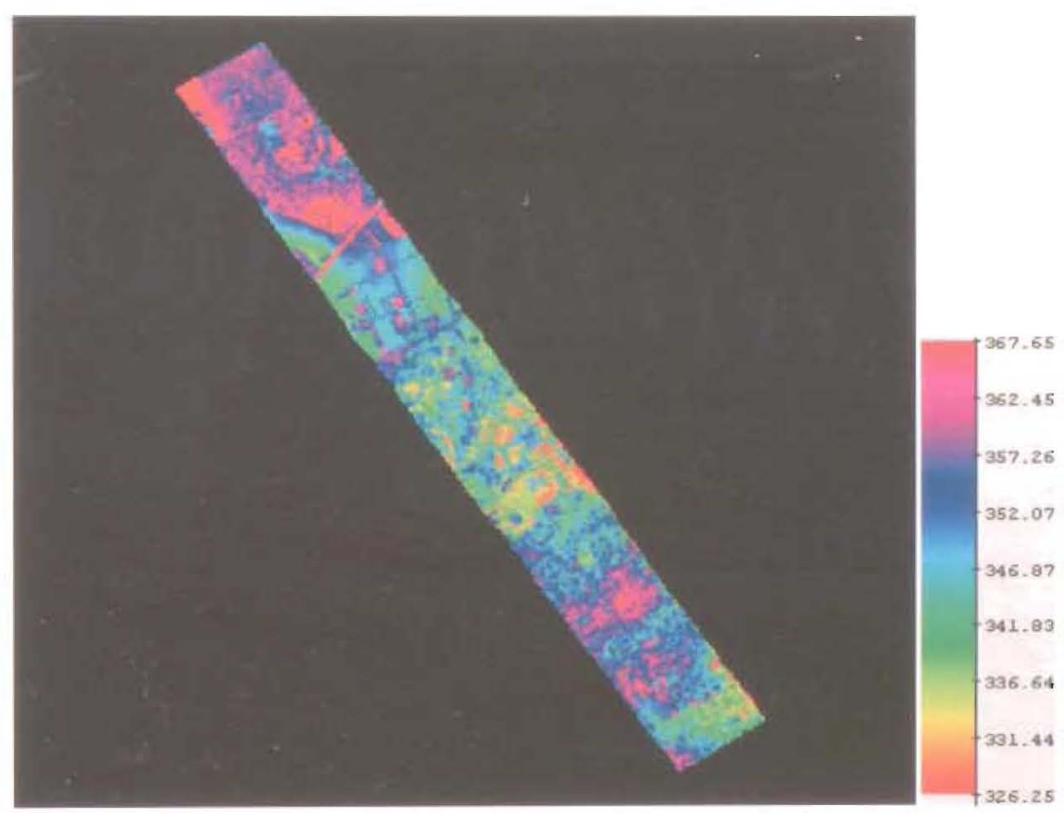

Figure 5.1 Topographic LiDAR point clouds of the University of Waterloo campus and surrounding area

Figure 5.2 demonstrates a reference map from Google map, the region in black rectangle is the proximate area covered by topographic LiDAR data. 


\section{RESULTS AND DISCUSSIONS}

\subsection{Study Area and Data}

The study area is a part of the University of Waterloo (UW) campus, covered by a single strip topographic LiDAR point clouds. Figure 5.1 exhibits the image and its legend. Different colours are utilized to symbolize the elevation of each point. The numbers are the absolute elevations in metres.

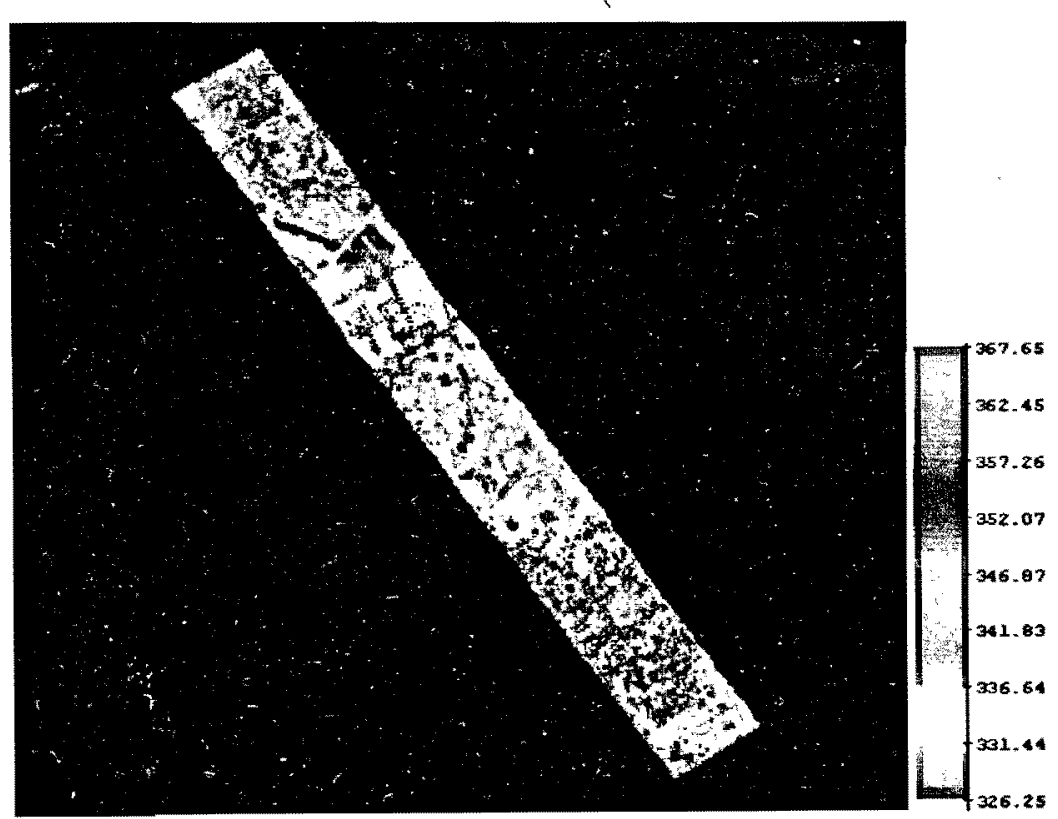

Figure 5.1 Topographic LiDAR point clouds of the University of Waterloo campus and

surrounding area

Figure 5.2 demonstrates a reference map from Google map, the region in black rectangle is the proximate area covered by topographic LiDAR data. 


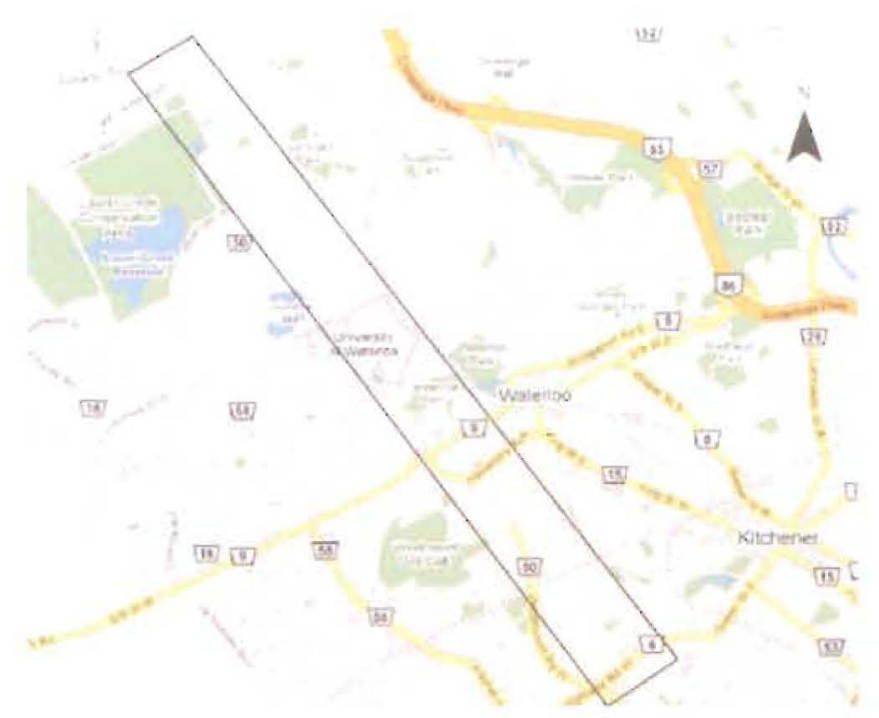

Figure 5.2 Reference map to topographic LiDAR image (Google map)

Table 5.1 lists relative topographic LiDAR data information and hardware settings when data was collected.

Table 5.1 Topographic LiDAR data specifications

\begin{tabular}{|l|c|}
\hline \multicolumn{1}{|c|}{ Coverage } & UW Campus and neighboring area \\
\hline Acquisition date & March 11,2006 \\
\hline No. of points & 7.9 million \\
\hline Scanner & ALTM GEMINI \\
\hline POS system & Applanix-POS/AV \\
\hline Flying height $(\mathrm{m} \mathrm{ASL})$ & 1200 \\
\hline Speed $(\mathrm{m} / \mathrm{s})$ & 66.9 \\
\hline Scan frequency $(\mathrm{Hz})$ & 35 \\
\hline Swath $(\mathrm{m})$ & 873.53 \\
\hline Desired resolution $(\mathrm{m})$ & 0.908 \\
\hline Point density $\left(\right.$ per $\left.\mathrm{m}^{2}\right)$ & 1.1 \\
\hline Data format & LAS \\
\hline Source & Optech Inc \\
\hline
\end{tabular}

Figure 5.3 displays part of header file from topographic LiDAR data where essential facts 


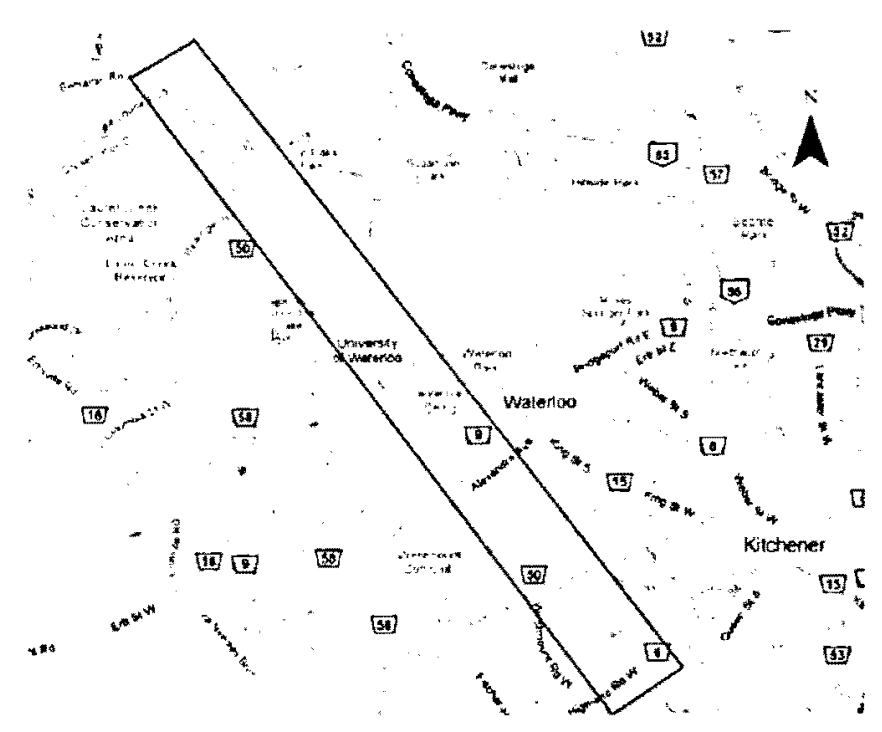

Figure 5.2 Reference map to topographic LiDAR image (Google map)

Table 5.1 lists relative topographic LiDAR data information and hardware settings when data was collected.

Table 5.1 Topographic LiDAR data specifications

\begin{tabular}{|l|c|}
\hline \multicolumn{1}{|c|}{ Coverage } & UW Campus and neighboring area \\
\hline Acquisition date & March 11, 2006 \\
\hline No. of points & 7.9 million \\
\hline Scanner & ALTM GEMINI \\
\hline POS system & Applanix-POS/AV \\
\hline Flying height $(\mathrm{m} \mathrm{ASL})$ & 1200 \\
\hline Speed $(\mathrm{m} / \mathrm{s})$ & 66.9 \\
\hline Scan frequency $(\mathrm{Hz})$ & 35 \\
\hline Swath $(\mathrm{m})$ & 873.53 \\
\hline Desired resolution $(\mathrm{m})$ & 0.908 \\
\hline Point density $\left(\right.$ per $\left.\mathrm{m}^{2}\right)$ & 1.1 \\
\hline Data format & LAS \\
\hline Source & Optech Inc \\
\hline
\end{tabular}

Figure 5.3 displays part of header file from topographic LiDAR data where essential facts 
about the dataset, including number of returned recorded, margin values, offsets and scale factors of $\mathrm{X}, \mathrm{Y}, \mathrm{Z}$ variables are recorded. They are critical when format conversion or filter operations are required.

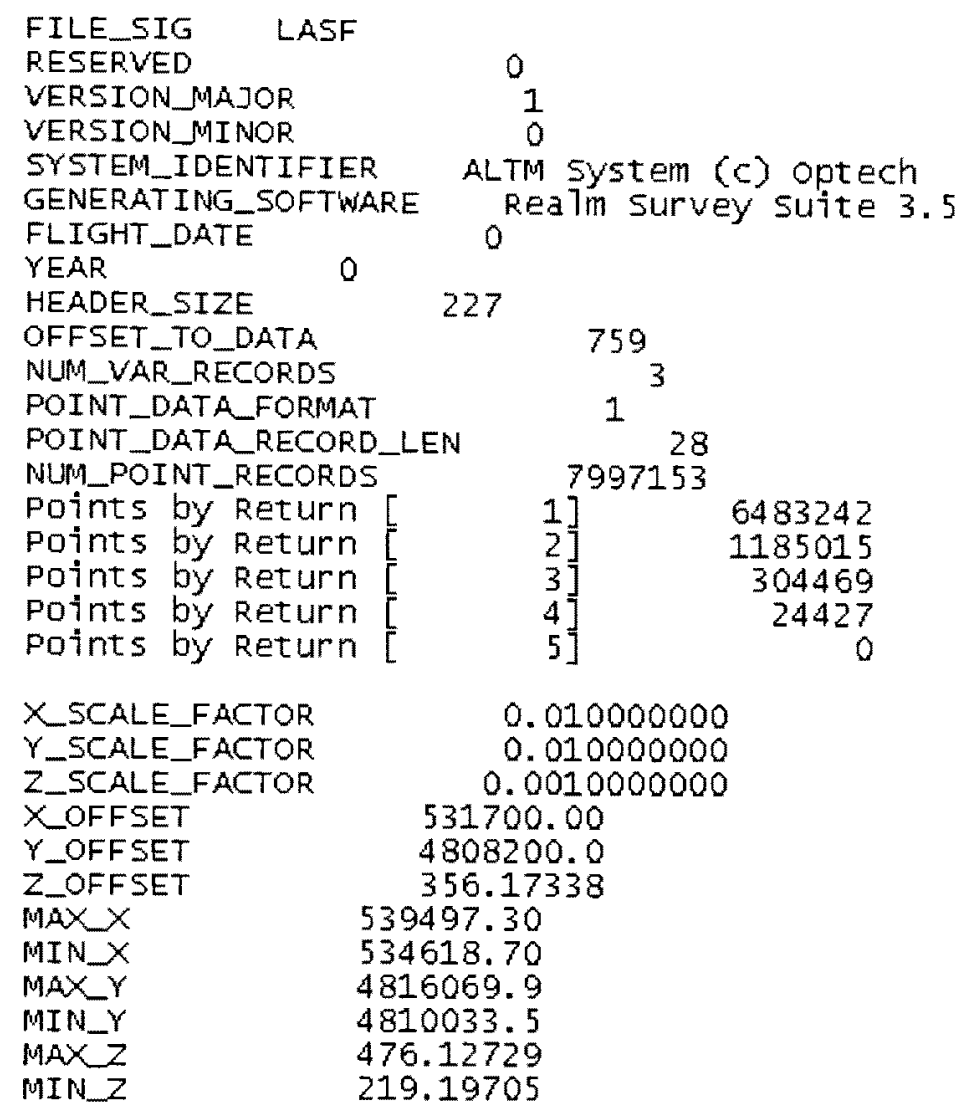

Figure 5.3 Part of header file

Figure 5.4 is aerial image of UW campus, which acts as reference image during the processing.

Buildings in coloured squares are candidates chosen to demonstrate the $3 \mathrm{D}$ building reconstruction process elaborated in previous chapter. In Table 5.2 the main properties of aerial image are listed. 


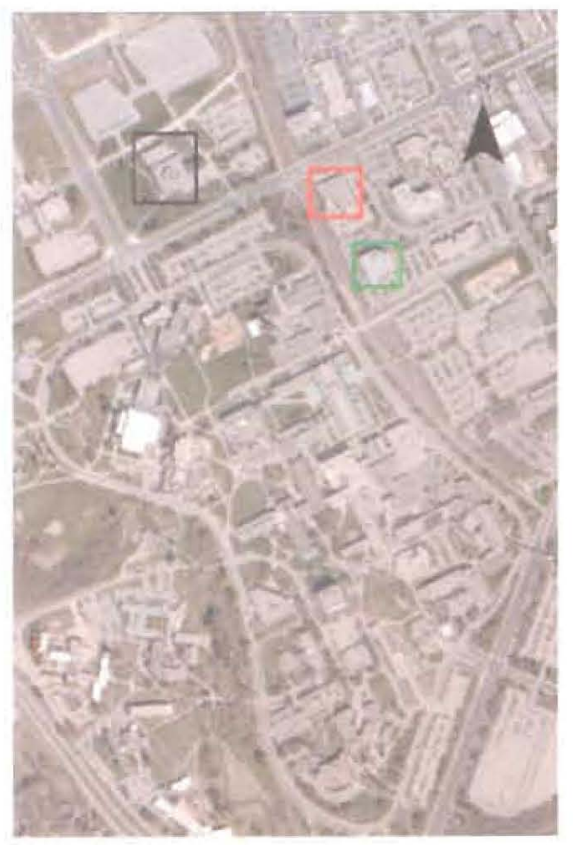

Figure 5.4 Aerial image of UW campus

Table 5.2 Aerial image specification

\begin{tabular}{|l|c|}
\hline \multicolumn{1}{|c|}{ Coverage } & University of Waterloo (UW) campus \\
\hline Format & GeoTIFF \\
\hline Resolution & $1 \mathrm{~m}$ \\
\hline Dimension & $1369 * 881$ \\
\hline Source & UW library \\
\hline
\end{tabular}

The study area is the UW northeast campus. The detailed description of individual building is given in Table 5.3. Buildings are aligned by complexity scale in increasing order. The common practices in $3 \mathrm{D}$ building reconstruction including convex polygons, concave polygons, curved edges, non-perpendicular edges, preliminary noise filtering techniques are examined in the study. 


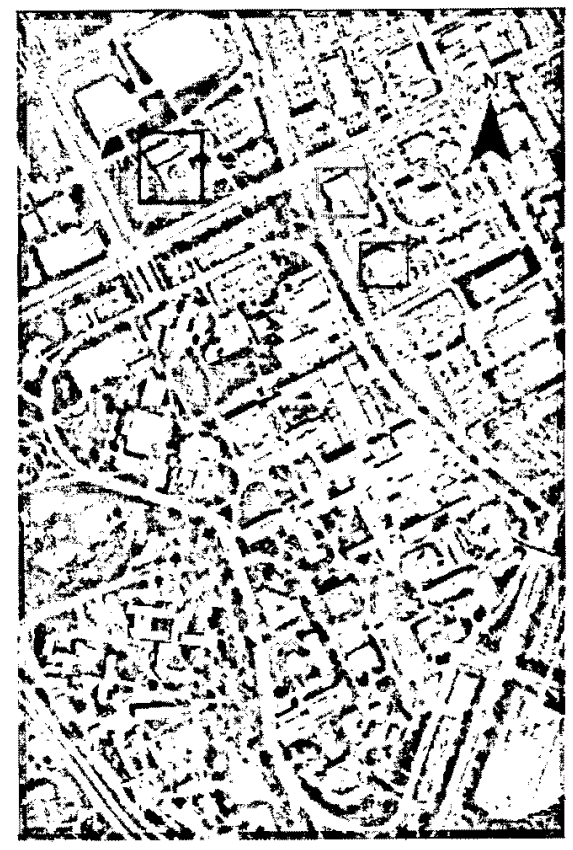

Figure 5.4 Aerial image of UW campus

Table 5.2 Aerial image specification

\begin{tabular}{|l|c|}
\hline \multicolumn{1}{|c|}{ Coverage } & University of Waterloo (UW) campus \\
\hline Format & GeoTIFF \\
\hline Resolution & $1 \mathrm{~m}$ \\
\hline Dimension & $1369 * 881$ \\
\hline Source & UW library \\
\hline
\end{tabular}

The study area is the UW northeast campus. The detailed description of individual building is given in Table 5.3. Buildings are aligned by complexity scale in increasing order. The common practices in $3 \mathrm{D}$ building reconstruction including convex polygons, concave polygons, curved edges, non-perpendicular edges, preliminary noise filtering techniques are examined in the study. 
Table 5.3 Sample building properties

\begin{tabular}{|c|c|c|}
\hline Building name & Aerial image & Building properties \\
\hline RIM & & $\begin{array}{l}\text { high-rise building, convex polygon } \\
\text { shape except for one curved edge, sparse } \\
\text { vegetation around. }\end{array}$ \\
\hline $\mathrm{BFG}$ & & $\begin{array}{l}\text { low-rise building, irregular concave } \\
\text { polygon shape, medium vegetation } \\
\text { presence close to building. }\end{array}$ \\
\hline Optometry & & $\begin{array}{l}\text { multi-level building complex, strong } \\
\text { appearance of vegetation. }\end{array}$ \\
\hline
\end{tabular}

The original topographic LiDAR point clouds contain 7.9 million points. In order to extract building roof outlines listed in Table 5.3, points covering the building areas need to be separated. As discussed in Chapter Two, each point in the topographic LiDAR point clouds is geo-referenced, usually in the UTM system. Two steps are involved to achieve the goal. First, spatial references of comer points need to be positioned. The reference image in Figure 5.4, provides desired spatial locations of corner points (lower left and upper right points).Next, points external to comer points are filtered out. LAStool (Isenburg and Shewchuk, 2008) contains basic functions handling topographic LiDAR data in LAS format and filtering and 
Table 5.3 Sample building properties

\begin{tabular}{|l|l|l|}
\hline Building name & Berial image & Building properties \\
\hline RIM & Ligh-rise building, convex polygon \\
shape except for one curved edge, sparse \\
vegetation around.
\end{tabular}

The original topographic LiDAR point clouds contain 7.9 million points. In order to extract building roof outlines listed in Table 5.3, points covering the building areas need to be separated. As discussed in Chapter Two, each point in the topographic LiDAR point clouds is geo-referenced, usually in the UTM system. Two steps are involved to achieve the goal. First, spatial references of corner points need to be positioned. The reference image in Figure 5.4, provides desired spatial locations of corner points (lower left and upper right points).Next, points external to corner points are filtered out. LAStool (Isenburg and Shewchuk, 2008) contains basic functions handling topographic LiDAR data in LAS format and filtering and 
format conversion functions are utilized in this study.

Figure 5.5 displays part of pre-processed topographic LiDAR data in ASCII format, there are three columns separated by space, first two columns ( $x$ and $y$ ) offer spatial reference of the point, and the third column (z) indicates its elevation.

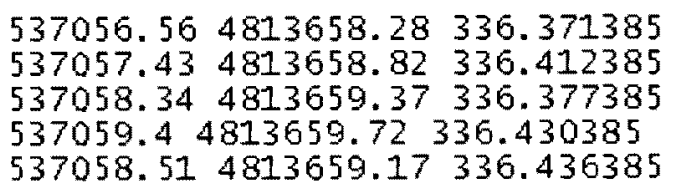

Figure 5.5 RIM.txt file

Figure 5.6 demonstrates the topographic LiDAR point clouds of sample buildings in an aerial view (Figures 5.6 (a), (c) and (e)) and 3D view (Figures 5.6 (b), (d) and (f)), respectively. The RIM building (Figures 5.6 (a) and (b)) has one level of flat roof, with Low Rise Vegetation (LRV) in the north part and low gradient ground. The BFG building (Figures 5.6 (c) and (d)) also has one level of flat roof, with close High Rise Vegetation (HRV) in the west and south part of the building and low topographic ground. The Optometry Building (Figures 5.6 (e) and (f)) has three levels of flat roofs of distinct altitude, there are heavy HRV surrounding the building except for north part, the ground surface is undulating. 


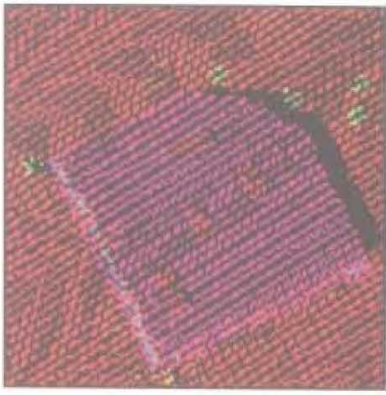

(a)

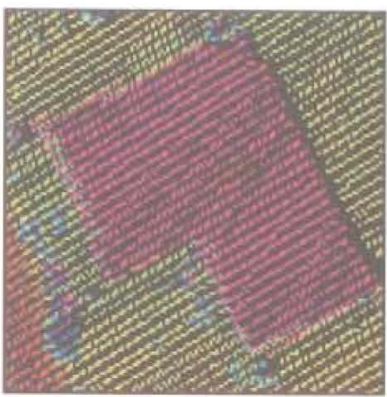

(c)

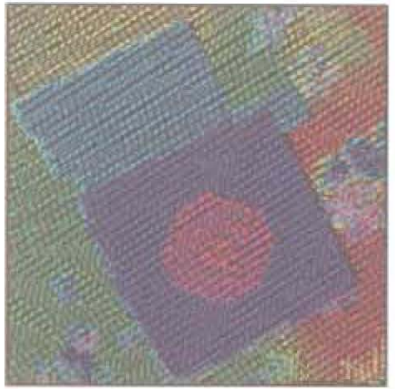

(e)

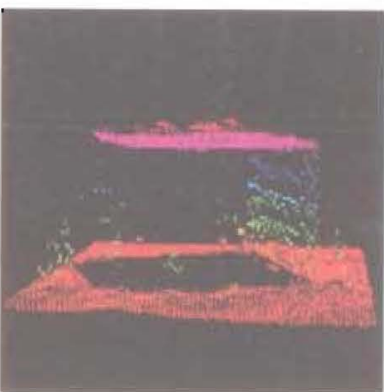

(b)

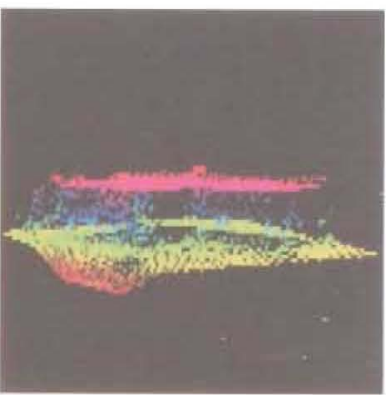

(d)

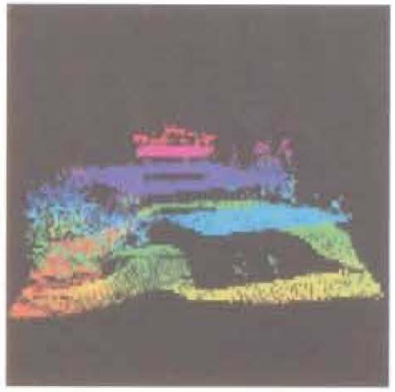

(f)

Figure 5.6 Topographic LiDAR Images of sample buildings

\subsection{Experiments}

\subsubsection{Segmentation}

Table 5.4 lists all constants for the segmentation algorithm presented in Section 4.1. The value $k$ is the number of groups into which a given topographic LiDAR data set is segmented, 


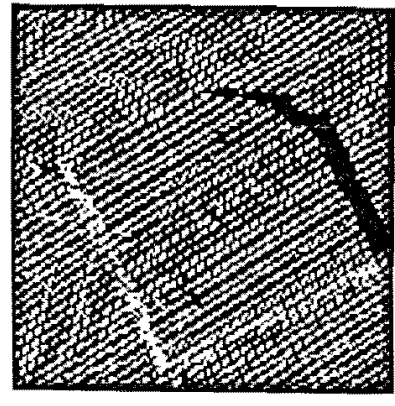

(a)

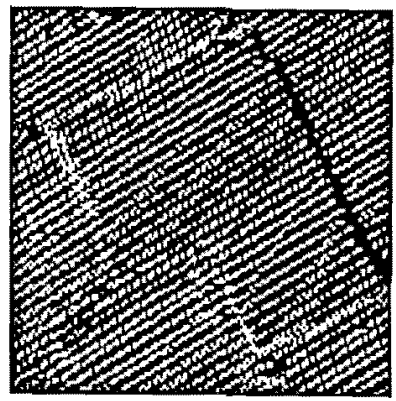

(c)

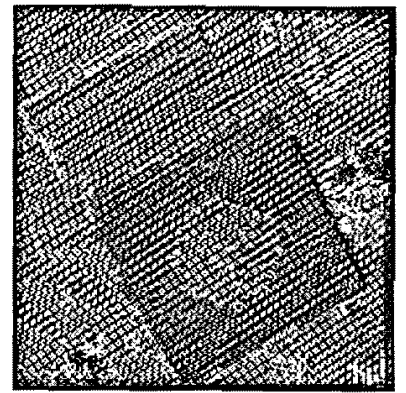

(e)

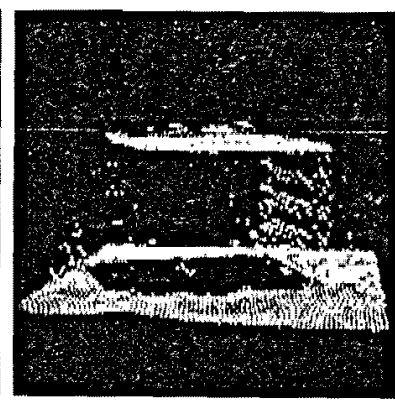

(b)

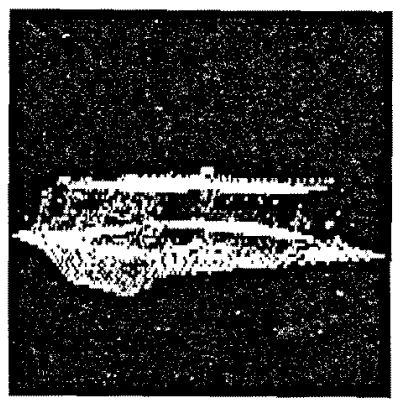

(d)

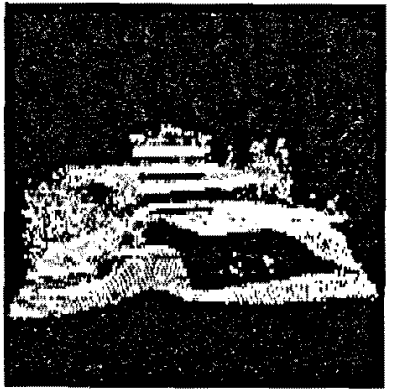

(f)

Figure 5.6 Topographic LiDAR Images of sample buildings

\subsection{Experiments}

\subsubsection{Segmentation}

Table 5.4 lists all constants for the segmentation algorithm presented in Section 4.1. The

value $k$ is the number of groups into which a given topographic LiDAR data set is segmented, 
prior knowledge about the scene covered by dataset is helpful. The variable $i$ represents the computing cycles. And $r$ is the size of neighbouring window for each point, the rule for selecting window is that there must be at least one neighbouring point in the window. The parameter $\sigma^{2}$ is the variance of the normal distribution corresponding to the elevations of points in each group and it controls the extent to which the elevations fluctuate around their means. Finally, $\varepsilon^{2}$ governs the span of the samples of the mean in successive iterations.

Table 5.4 Input constants

\begin{tabular}{|l|l|}
\hline \multicolumn{1}{|c|}{ Name } & Abbreviation \\
\hline Radius & $r$ \\
\hline Number of groups & $k$ \\
\hline Variance of each group & $\sigma^{2}$ \\
\hline Variance of mean & $\varepsilon^{2}$ \\
\hline Number of iterations & $i$ \\
\hline
\end{tabular}

Table 5.5 lists constants of the three scenes. The value of $r$ is related to the desired resolution in Table 5.1 and it should be slightly larger than the value of desired resolution to ensure that each point has at least one neighbouring point. The parameter $k$ symbolizes the segment number that the data is divided into. Scene 1 contains ground and building roof and 2 is picked. Scene 2 consists of ground, HRV and building roof and 3 is selected. Scene 3 includes three levels of building roofs and three levels of ground surfaces, so 6 is chosen. The choice of $\sigma^{2}$ and $\varepsilon^{2}$ requires some prior knowledge about the scene. For instance, RIM building has low rise vegetation around so larger $\sigma^{2}\left(2 \mathrm{~m}^{2}\right)$ is selected, in order to reduce computing time, move mean of each group its stable value, $2 \mathrm{~m}^{2}$ is chosen for $\varepsilon^{2}$ so its mean 
values will move faster to their stable values. The experiment shows that as $k$ increases, both $\sigma^{2}$ and $\varepsilon^{2}$ should be reduced to achieve optimal solution. The choice of $i$ is linked with the $k$ and $\varepsilon^{2}$, as $k$ increases and $\varepsilon^{2}$ decreases, larger $i$ value is expected. The selection of $i$ in this case is based on the step-wised experiment.

Table 5.5 Parameters of sample buildings

\begin{tabular}{|l|l|l|l|}
\hline Constants & \multicolumn{1}{|c|}{ Scene 1} & \multicolumn{1}{|c|}{ Scene 2} & \multicolumn{1}{c|}{ Scene 3 } \\
\hline$r$ & $1.1 \mathrm{~m}$ & $1.1 \mathrm{~m}$ & $1.1 \mathrm{~m}$ \\
\hline$k$ & 2 & 3 & 6 \\
\hline$\sigma^{2}$ & $2 \mathrm{~m}^{2}$ & $1.5 \mathrm{~m}^{2}$ & $1 \mathrm{~m}^{2}$ \\
\hline$\varepsilon^{2}$ & $2 \mathrm{~m}^{2}$ & $1.5 \mathrm{~m}^{2}$ & $1 \mathrm{~m}^{2}$ \\
\hline$i$ & 200,000 & 150,000 & 850,000 \\
\hline
\end{tabular}

Figure 5.7 shows the process of segmentation of Scene 1. Figure 5.7 (a) demonstrates the initial state, where each point is assigned a group value randomly. After 50,000 iterations, points with similar elevation value ( $\mathrm{z}$ value) start to cluster together, which is shown in Figure 5.7 (b). The majority of points are segmented successfully after 100,000 iterations as in Figure 5.7 (c). Figure 5.7 (d) exhibits shows the final results of the segmentation procedure, all the points are divided into two groups, ground points (blue colour) and building points (green colour). It is noticed that there are a few blue points mixed into green points. This is caused by the ground returns when LiDAR beams go through building windows. Those noise points can be deleted by post-processing method and will not have effect for building boundary extraction. 


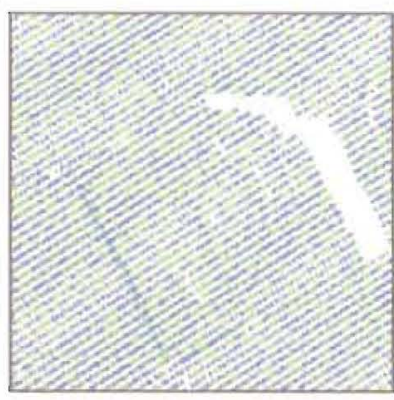

(a)

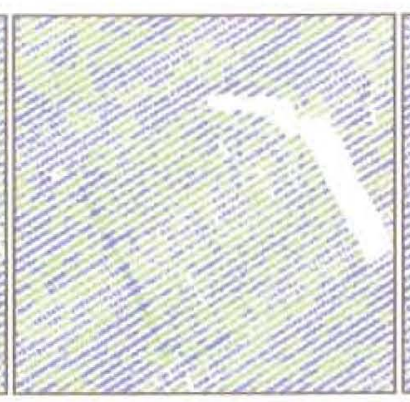

(b)

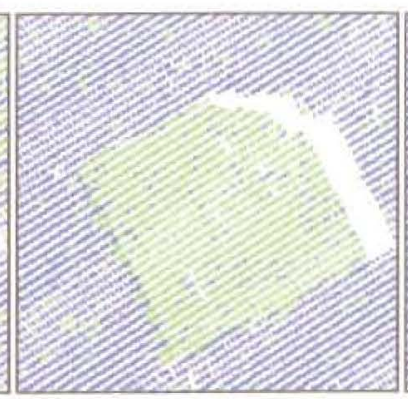

(c)

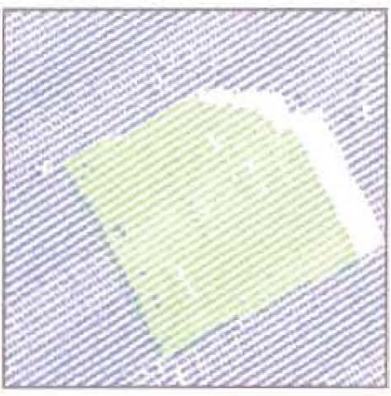

(d)

Figure 5.7 Segmentation of scene one

Figure 5.8 demonstrates the Acceptance Rate (AR) of $k$ : one means $k$ is accepted, zero otherwise. Figure 5.8 (a) reveals overall AR during complete computing cycle. Figures 5.8

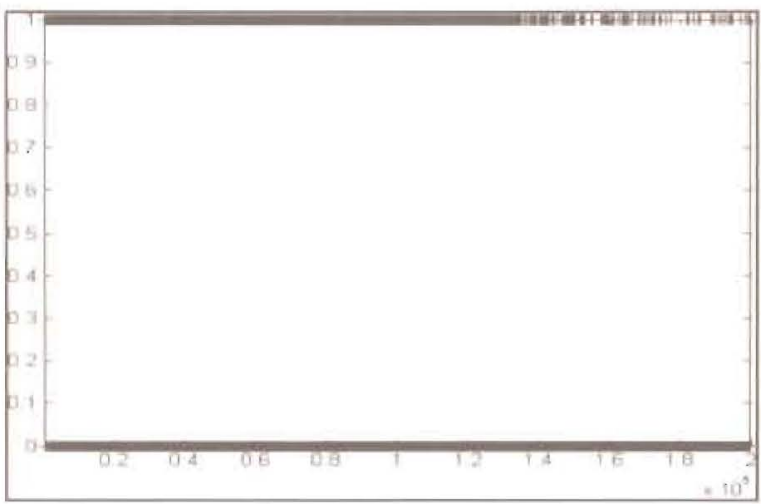

(a)

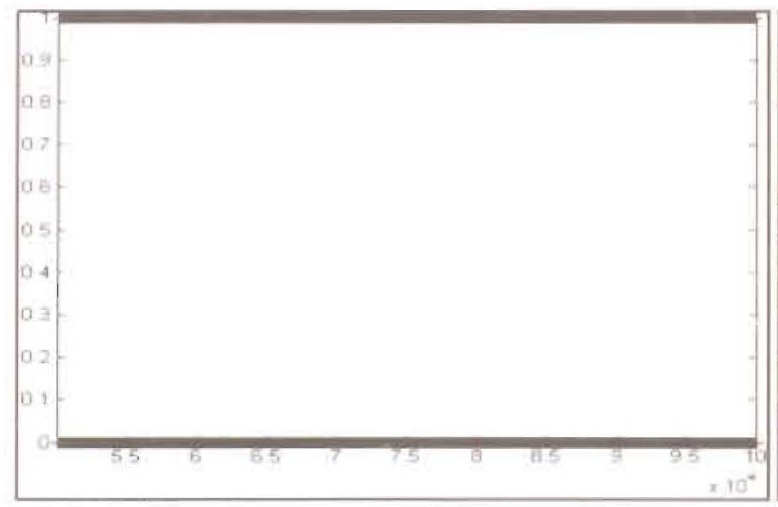

(c)

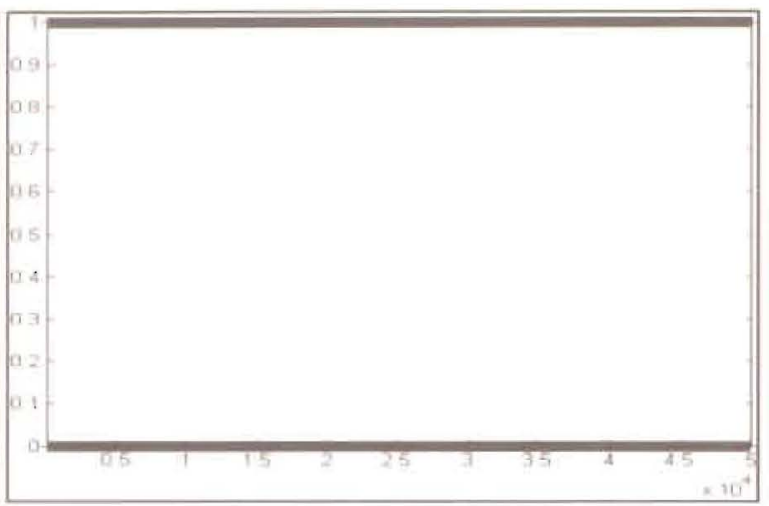

(b)

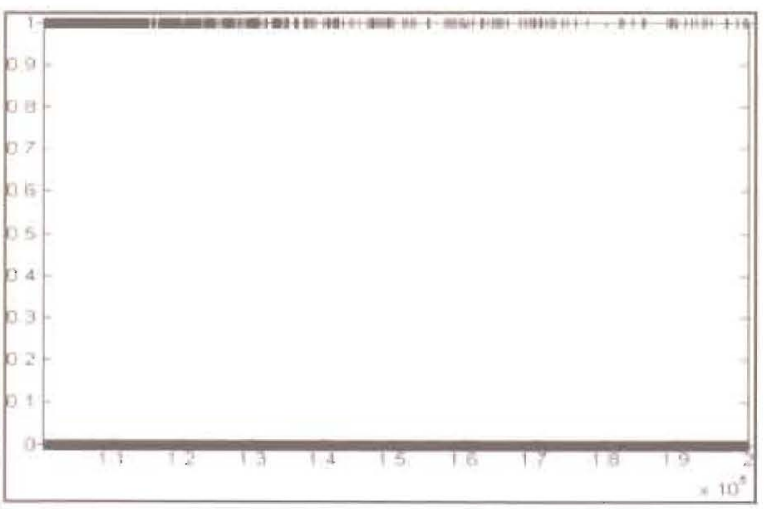

(d)

Figure 5.8 Acceptance rate of $k$

(b), (c) and (d) offer AR in first 50,000 repetitions, 50,000 to 100,000 repetitions and 100,000 


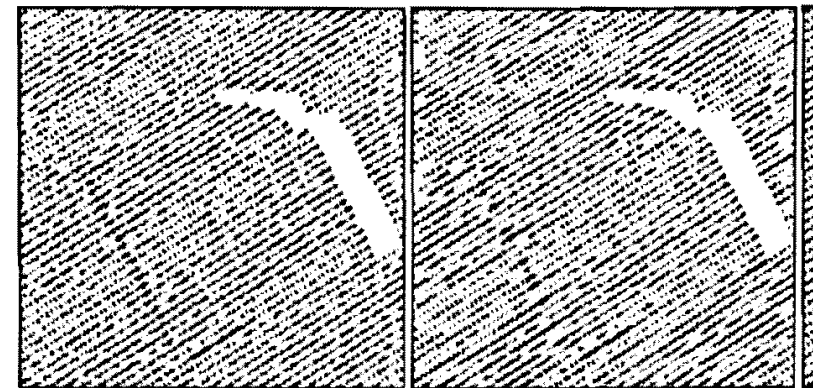

(a)

(b)

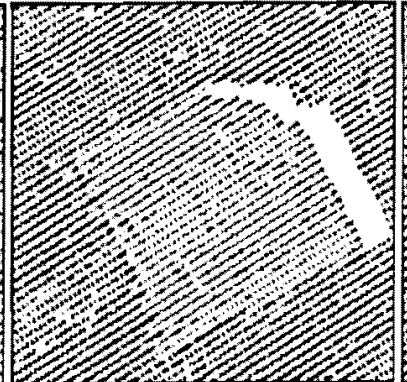

(c)

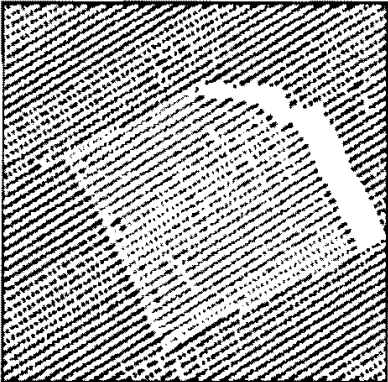

(d)

Figure 5.7 Segmentation of scene one

Figure 5.8 demonstrates the Acceptance Rate (AR) of $k$ : one means $k$ is accepted, zero otherwise. Figure 5.8 (a) reveals overall AR during complete computing cycle. Figures 5.8

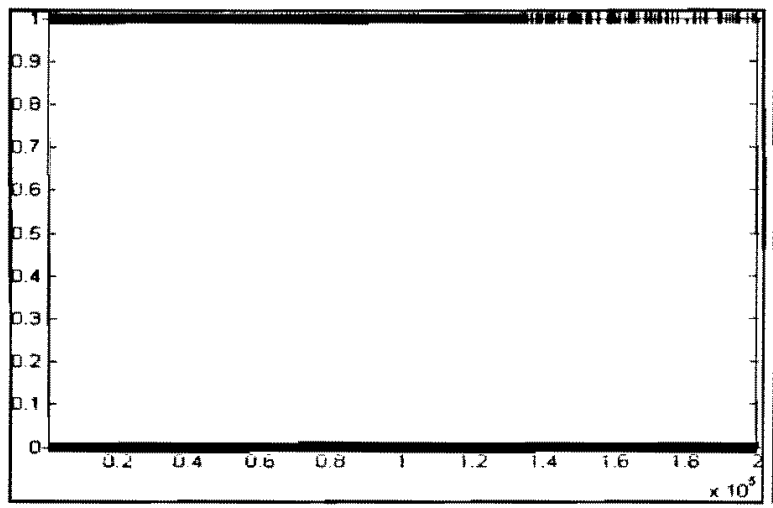

(a)

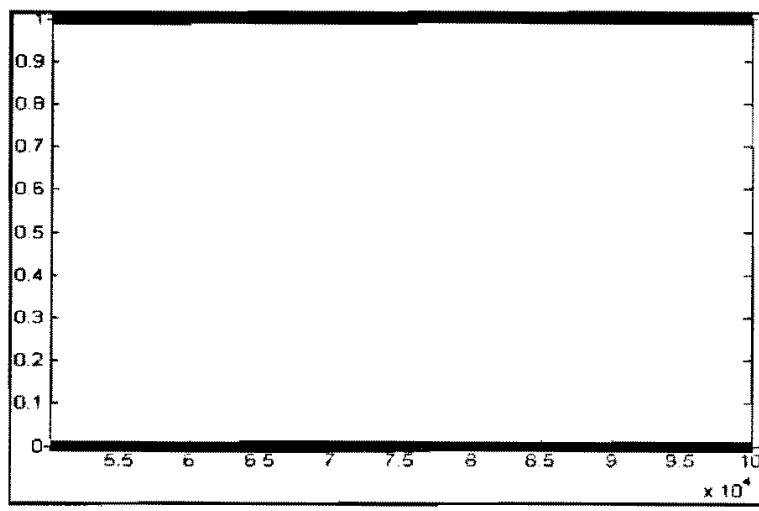

(c)

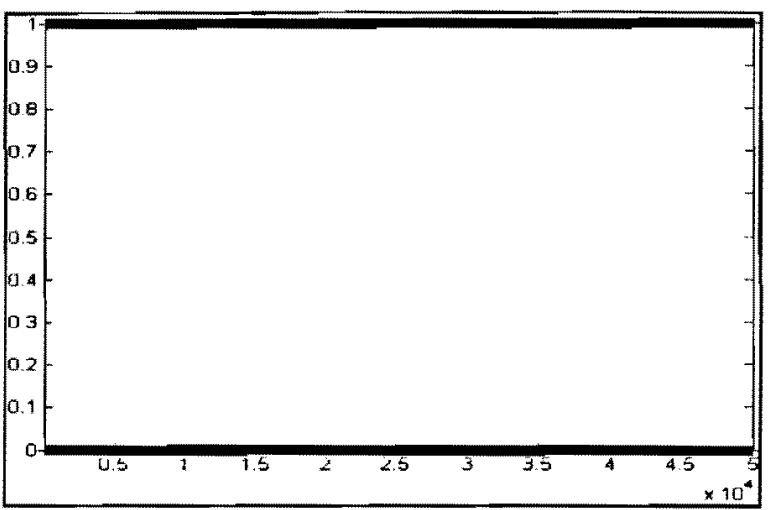

(b)

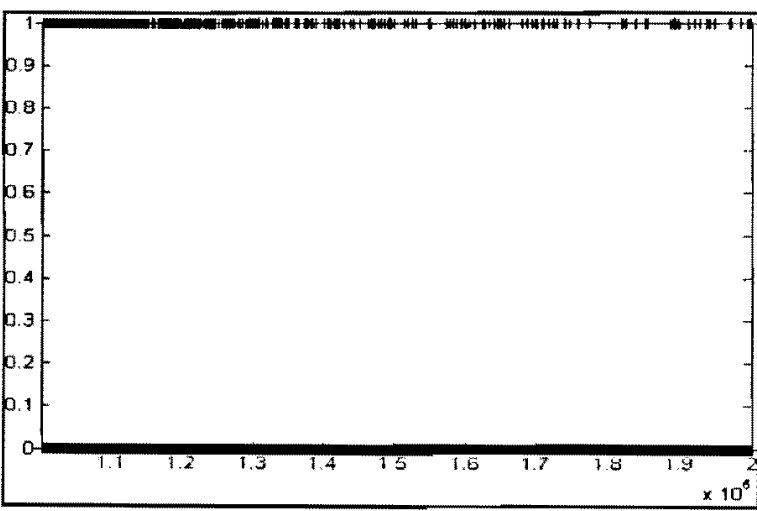

(d)

Figure 5.8 Acceptance rate of $k$

(b), (c) and (d) offer AR in first 50,000 repetitions, 50,000 to 100,000 repetitions and 100,000 
to 200,000 repetitions, respectively. AR in Figures 5.8 (b) and (c) are higher because of frequent label shifting rate and Figure 5.7 (b) and (c) show the same tendency. As most points are correctly labeled, new labels tend to be rejected and AR drops significantly, which is explained in Figure 5.8 (d).

Figure 5.9 shows the change of means for two groups during complete iterations, respectively. Figure 5.9 (a) shows mean shift of ground points. At the beginning a value is assigned arbitrarily between the minimum and maximum heights, as point labels switch, its mean value alters accordingly. Because a large portion of points are ground points and big elevation difference between ground points and building roof points, the mean value fluctuates dramatically before it stabilizes. Figure 5.9 (b) reflects the mean change of building roof points, which share similarities with Figure 5.9 (a).

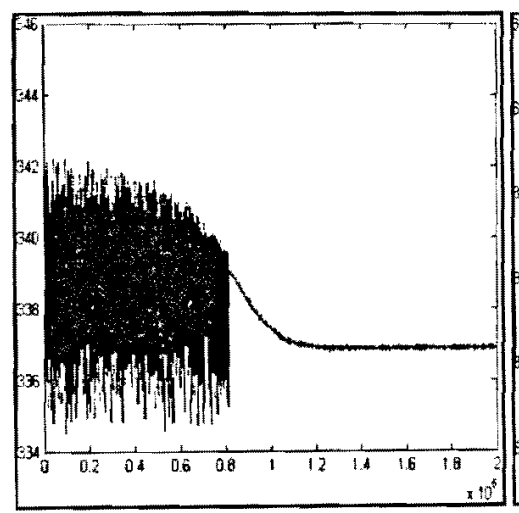

(a)

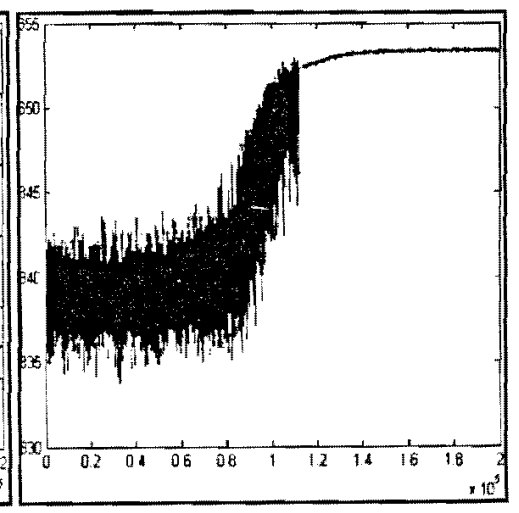

(b)

Figure $5.9 \mu$ Values against iteration

Figure 5.10 discloses the AR of mean for ground points, one means value is accepted and zero otherwise. Figure 5.10 (a) gives overall AR during whole computing procedure. Two 
thousand iterations are extracted from beginning, middle and end respectively to deliver better view of tendency, which are shown in Figures 5.10 (b), (c) and (d). With reference to Figure $5.9(\mathrm{a})$, at the beginning AR is high, from the middle of iterations and thereafter, $\mu_{I}$ decreases and becomes stable, only values close to its current mean are accept, which are reflected in Figures 5.10 (c) and (d).

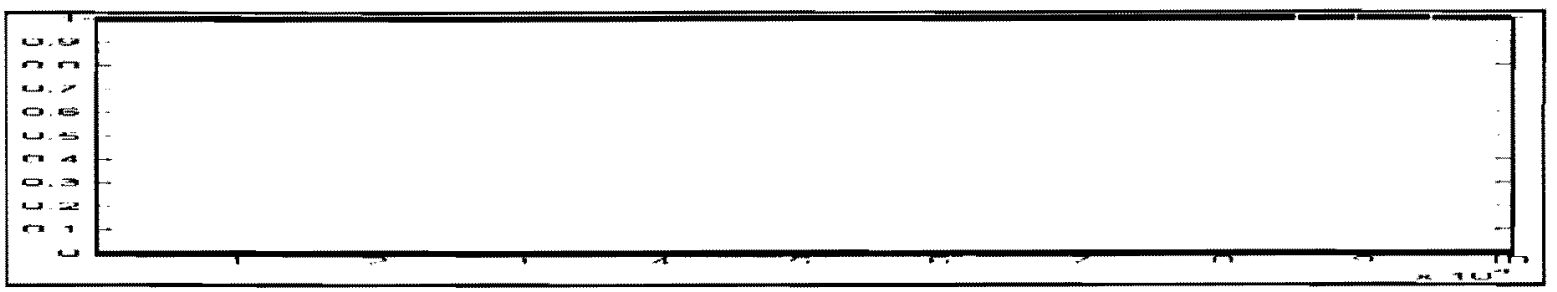

(a)

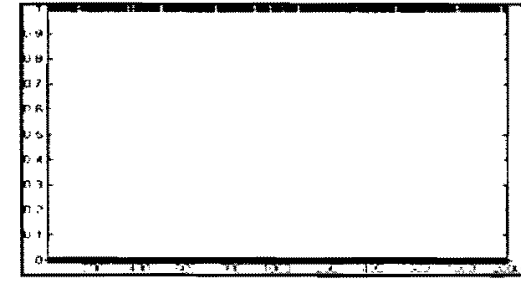

(b)

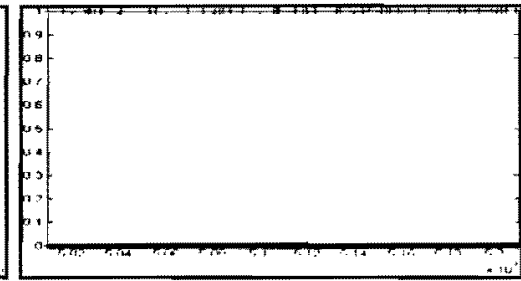

(c)

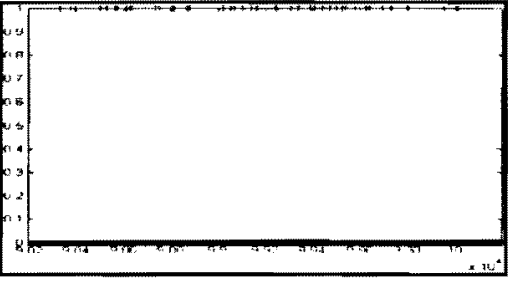

(d)

Figure 5.10 Acceptance rate of $\mu_{l}$

Figure 5.11 depicts the AR of mean for building roof points, which shows similar situation as in Figure 5.10 except that stability occurs after passing the middle of iterations. In Figure 5.11 (c), the mean is stilling changing (values shown in Figure 5.9 (b)), the stabilized situation is shown Figure 5.11 (d). 


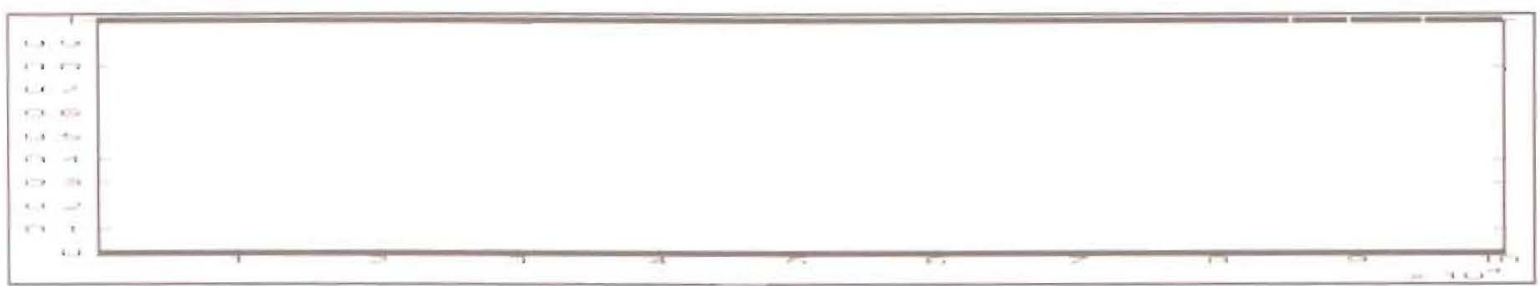

(a)

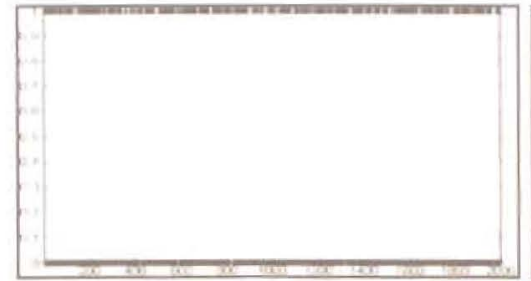

(b)

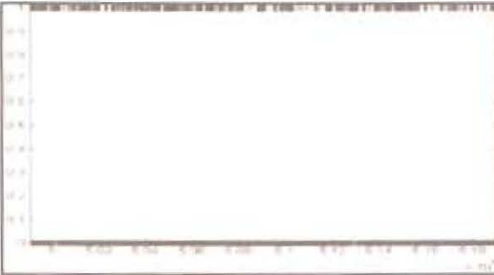

(c)

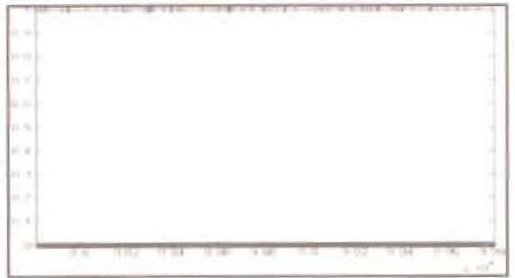

(d)

Figure 5.11 Acceptance rate of $\mu_{2}$

Figure 5.12 illustrates the segmentation operation of Scene 2. At beginning each point is assigned a label as shown in Figure 5.12 (a). Figure 5.12 (b) exhibits how points are grouped after 50,000 iterations, most points have aggregated properly. Figures 5.12 (c) and (d) display the results after 100,000 and 150,000 iterations respectively. By the end of operation, dataset is segmented into building roof layer (red), ground layer (blue) and vegetation layer (green and minor red points).

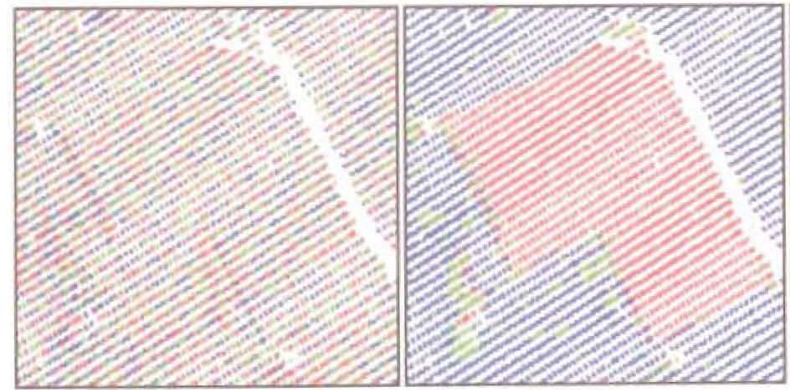

(a) (b)

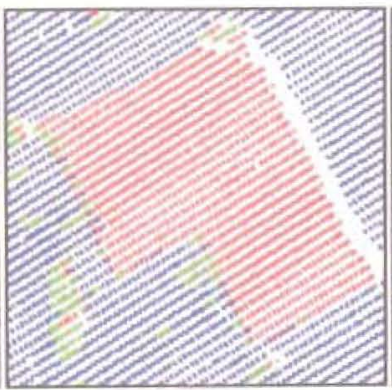

(c)

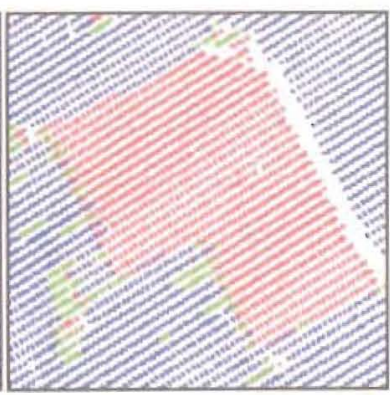

(d)

Figure 5.12 Segmentation of scene two 


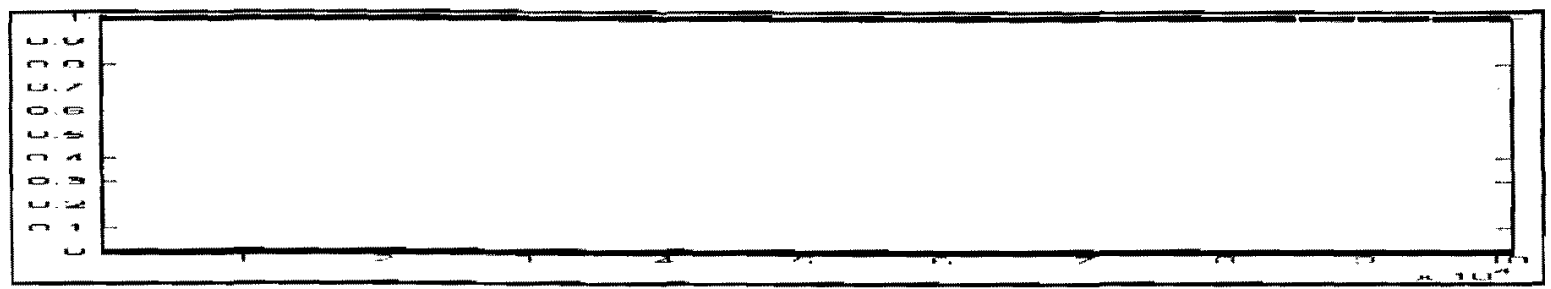

(a)

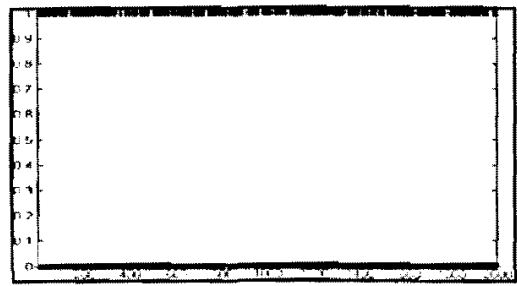

(b)

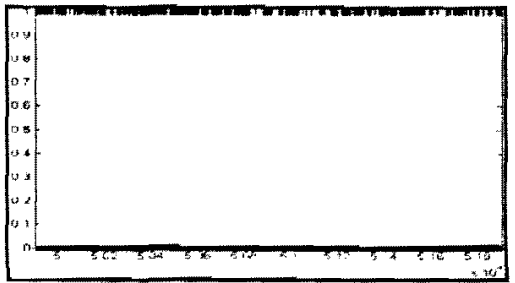

(c)

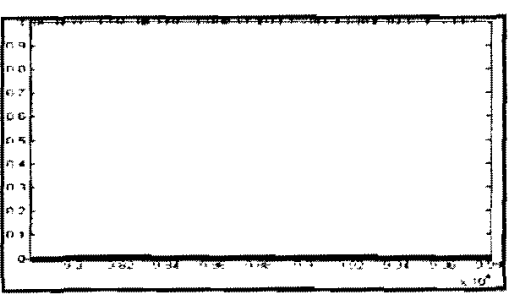

(d)

Figure 5.11 Acceptance rate of $\mu_{2}$

Figure 5.12 illustrates the segmentation operation of Scene 2. At beginning each point is assigned a label as shown in Figure 5.12 (a). Figure 5.12 (b) exhibits how points are grouped after 50,000 iterations, most points have aggregated properly. Figures 5.12 (c) and (d) display the results after 100,000 and 150,000 iterations respectively. By the end of operation, dataset is segmented into building roof layer (red), ground layer (blue) and vegetation layer (green and minor red points).

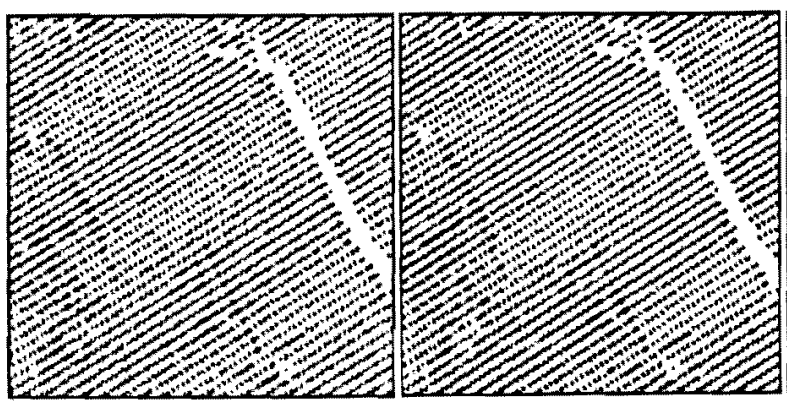

(a) (b)

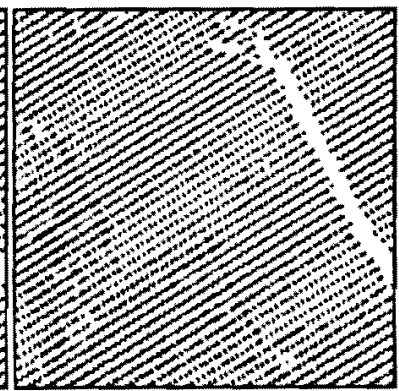

(d)

Figure 5.12 Segmentation of scene two 
Figure 5.13 shows the AR of $k$, one means label is accepted, zero otherwise. Figure 5.13 (a) offers AR in overall view. Figure 5.13 (b) shows AR during first 50,000 iterations, whose AR is the highest among three stages. AR declines gradually as computing advances to the 100,000 and 150,000 iterations, the tendency is revealed in Figures 5.13 (c) and (d), respectively. Because the majority of points are correctly labeled, new labels to these points are more likely to be rejected.

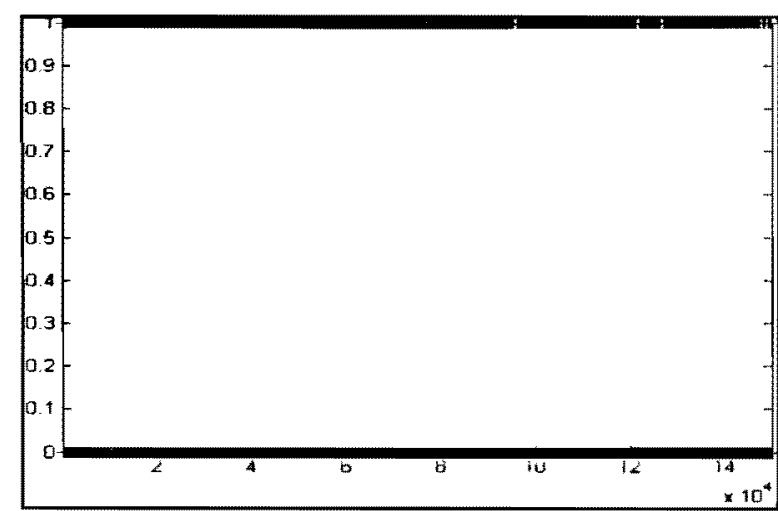

(a)

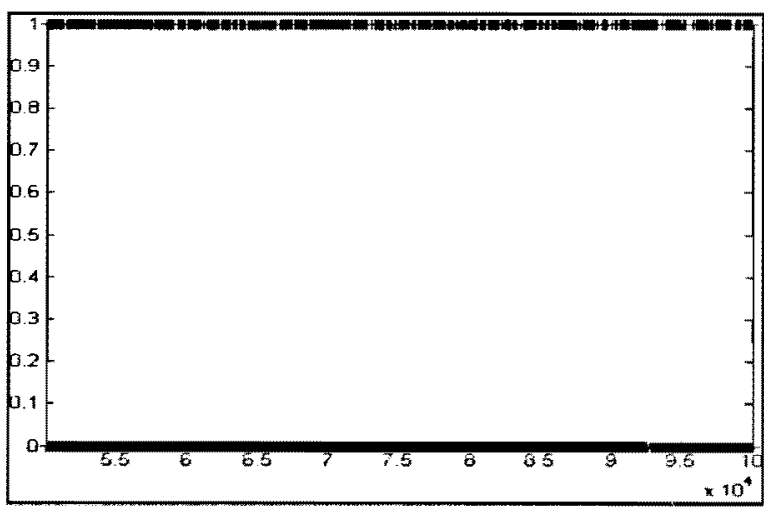

(c)

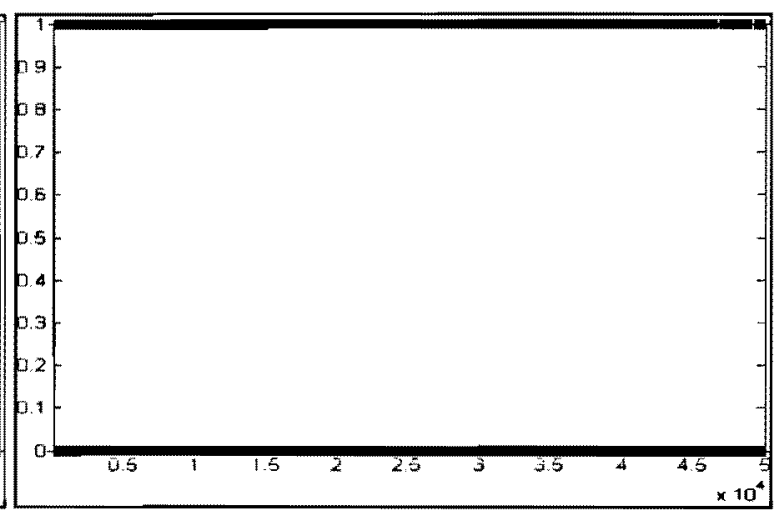

(b)

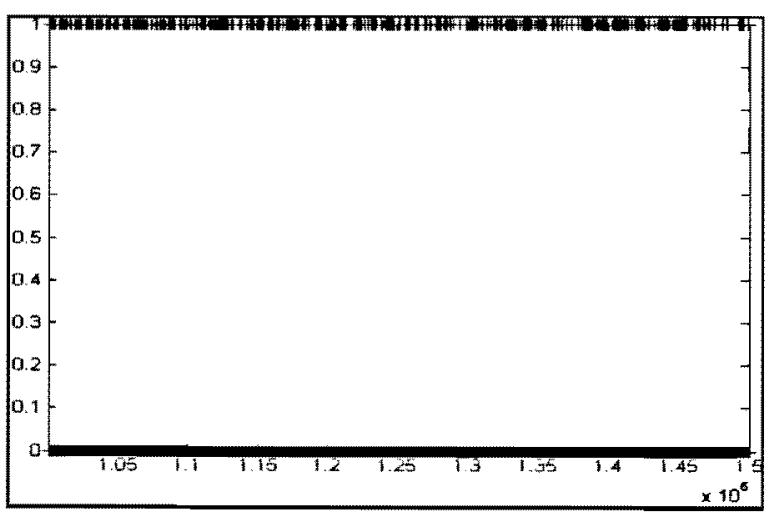

(d)

Figure 5.13 Acceptance rate of $k$

Figure 5.14 shows the mutation of means. Figure 5.14 (a) is for ground points, Figures 5.14 (b) and (c) are for vegetation and building roof points, respectively. Values in Figures 5.14 (a) 
and (c) become constant before 50,000 computing cycles, which is echoed by Figure 5.12 (b). Values in Figure 5.14 (b) oscillate in a wider range, because some points can be classified as either ground points or LRV points. Only a small portion of points are vegetation points, so the label changes have bigger impact on mean values.

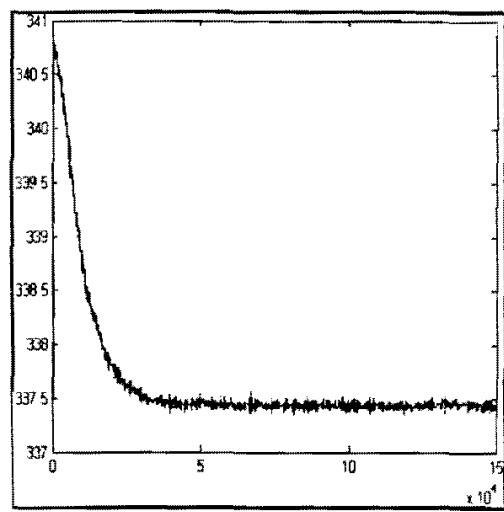

(a)

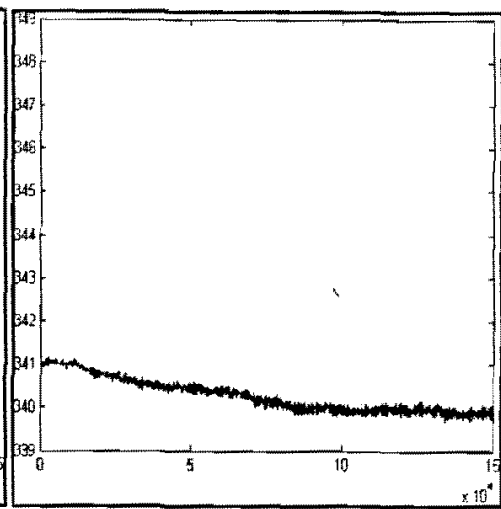

(b)

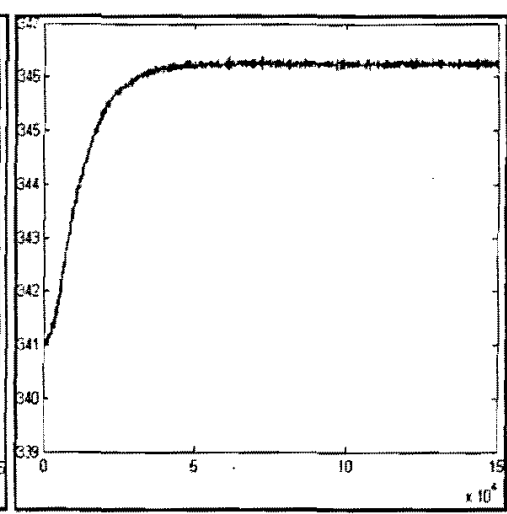

(c)

Figure $5.14 \mu$ Values against iteration

Figure 5.15 shows mean AR for ground points; one symbolizes acceptance and zero means

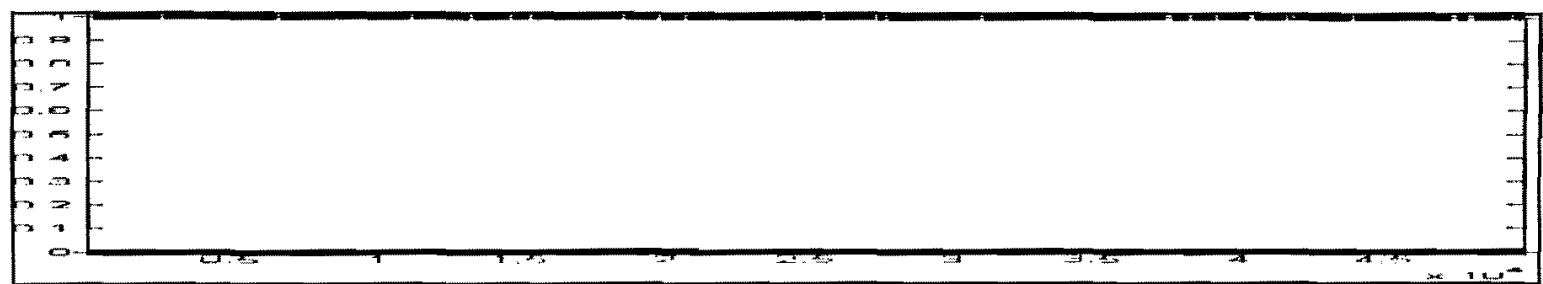

(a)

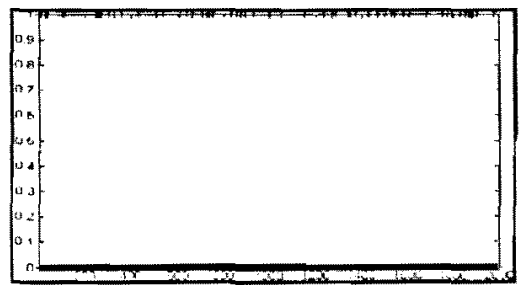

(b)

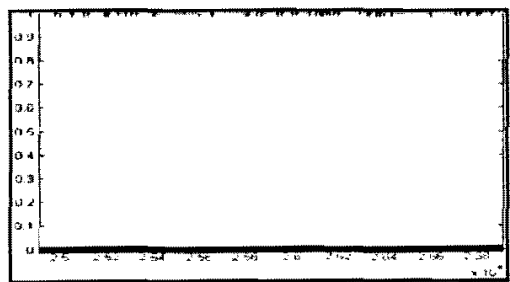

(c)

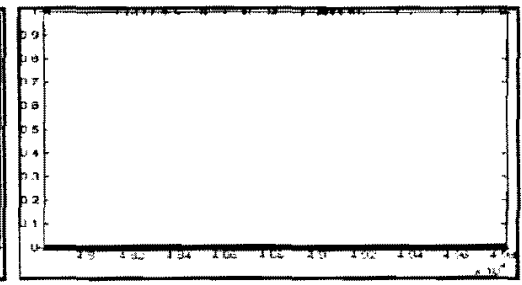

(d)

Figure 5.15 Acceptance rate of $\mu_{l}$ 
rejection. Figure 5.15 (a) shows overall AR. Figures 5.15 (b), (c) and (d) magnify two thousand iteration intervals from the beginning, middle and end part, respectively. It is clearly shown that AR declines as the calculation continues.

Figure 5.16 displays the mean AR for vegetation points. In contrary to what is shown in Figure 5.15, its value does not drop significantly as iteration develops, this is because label switches between ground points and LRV points happen frequently throughout the whole cycle, Figure 5.14 (b) indicates the same situation.

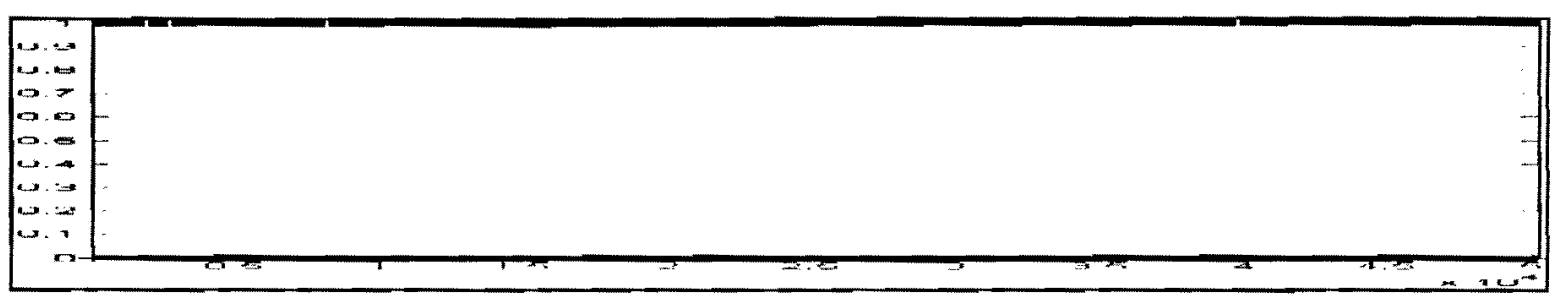

(a)

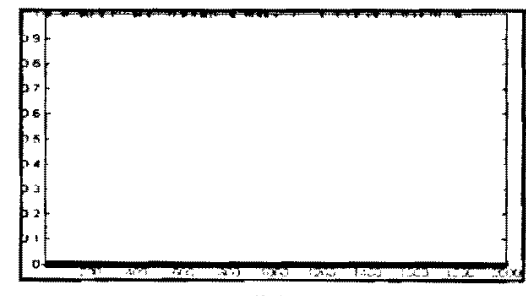

(b)

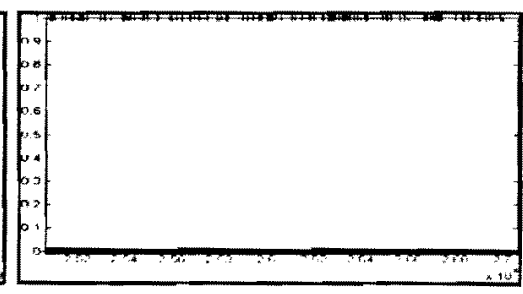

(c)

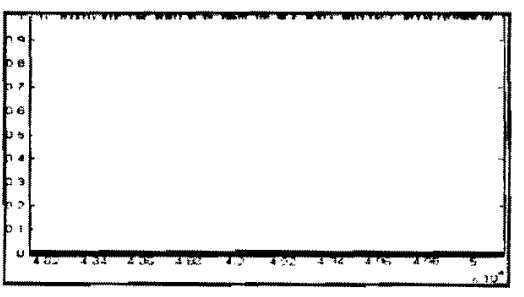

(d)

Figure 5.16 Acceptance rate of $\mu_{2}$

Figure 5.17 demonstrates the mean AR for building roof points. Its change shares similarities with that of ground points (shown in Figure 5.15). 


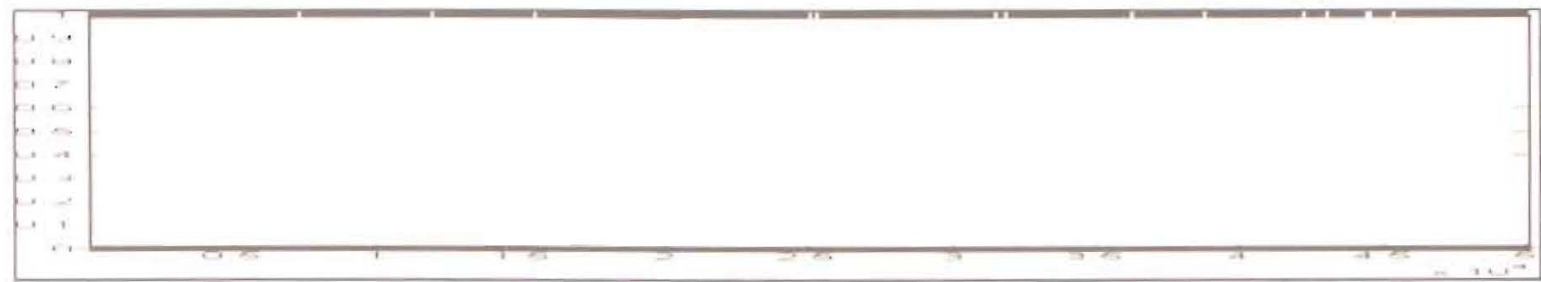

(a)

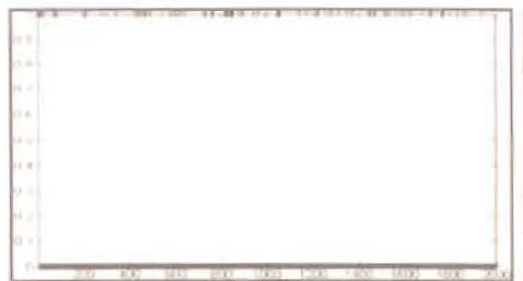

(b)

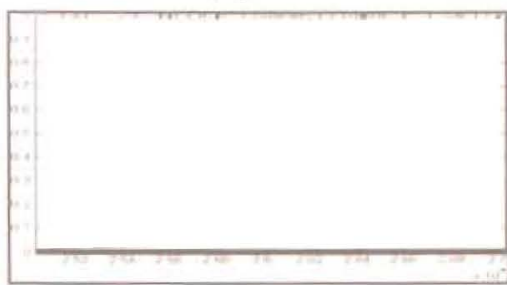

(c)

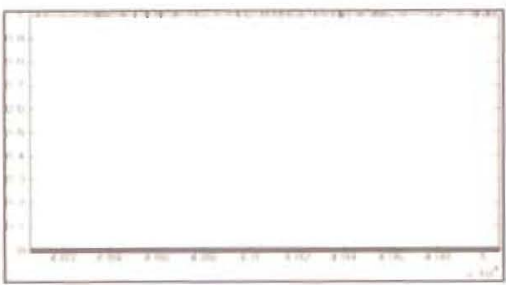

(d)

Figure 5.17 Acceptance rate of $\mu_{3}$

Figure 5.18 exhibits the segmentation process of Scene 3. At the initial stage, each point is allocated to one of the six labels randomly, which is shown in Figure 5.18 (a). After 150,000 iterations, the approximate shape of building and its surrounding area become clear as in Figure 5.18 (b). At the moment when 550,000 repetitions are completed (indicated in

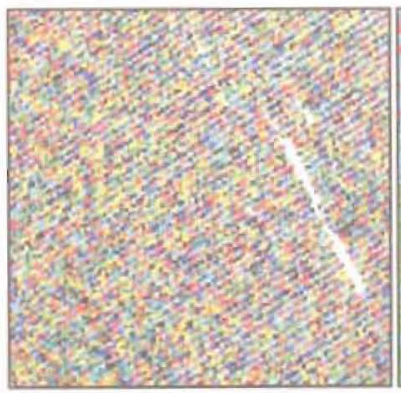

(a)

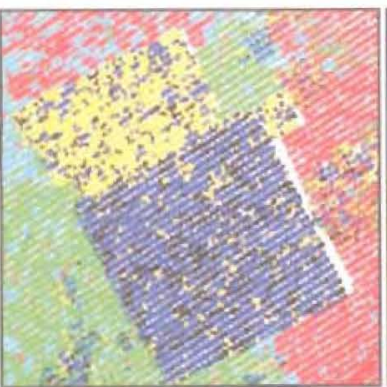

(b)

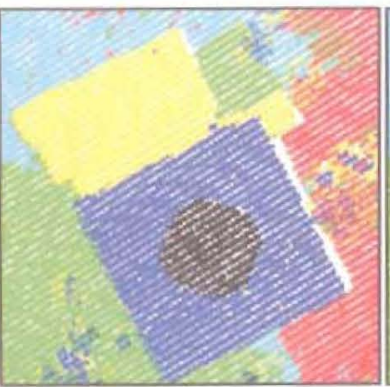

(c)

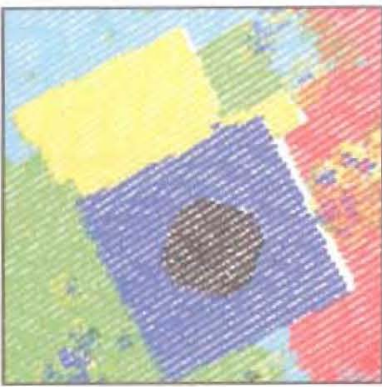

(d)

Figure 5.18 Segmentation of scene three

Figure 5.18(c)), six layers are clearly separated except for minor points which need label adjustment. Figure 5.18 (d) depicts the final result after 850,000 iterations. The dataset is divided into two categories: ground and building roof, ground category contains points in red 


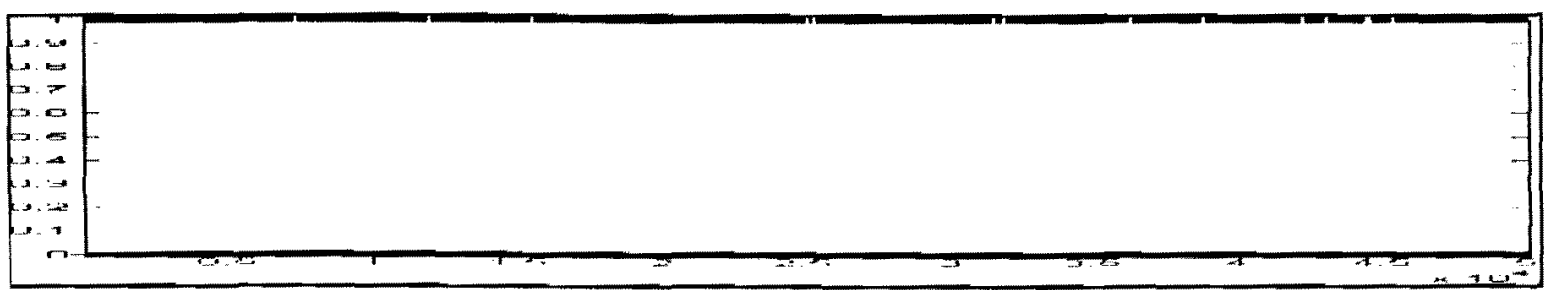

(a)

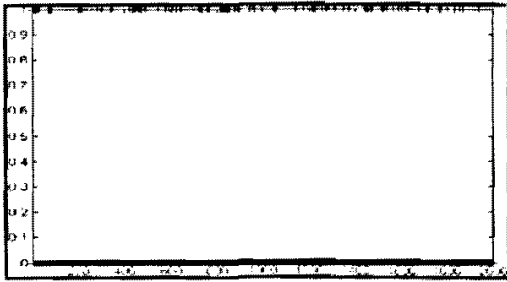

(b)

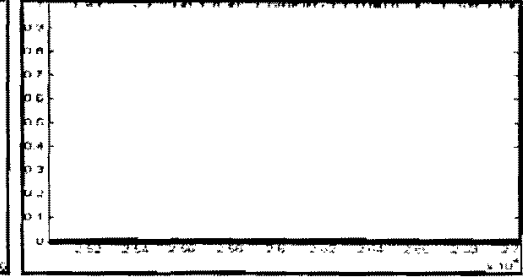

(c)

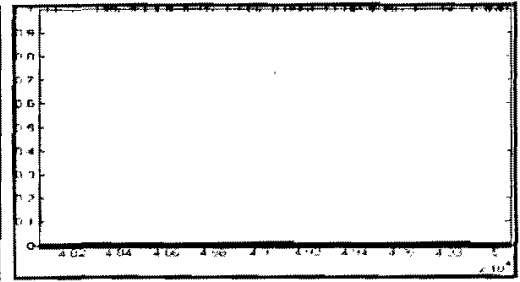

(d)

Figure 5.17 Acceptance rate of $\mu_{3}$

Figure 5.18 exhibits the segmentation process of Scene 3 . At the initial stage, each point is allocated to one of the six labels randomly, which is shown in Figure 5.18 (a). After 150,000 iterations, the approximate shape of building and its surrounding area become clear as in Figure 5.18 (b). At the moment when 550,000 repetitions are completed (indicated in

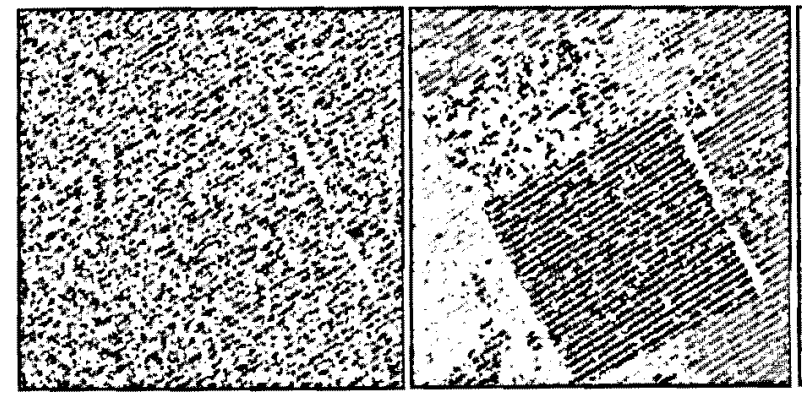

(a) (b)

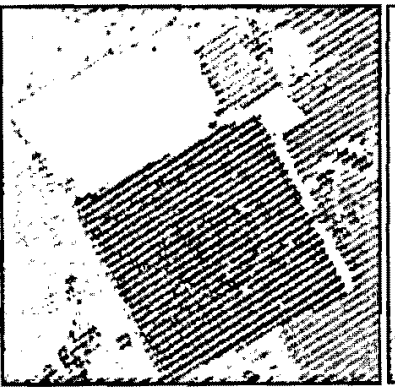

(c)

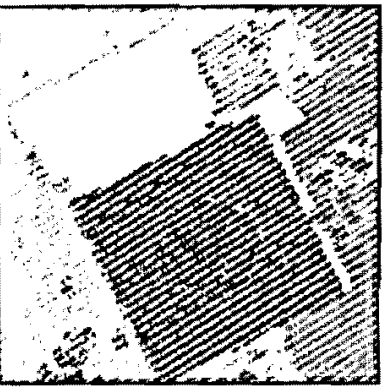

(d)

Figure 5.18 Segmentation of scene three

Figure 5.18(c)), six layers are clearly separated except for minor points which need label adjustment. Figure 5.18 (d) depicts the final result after 850,000 iterations. The dataset is divided into two categories: ground and building roof, ground category contains points in red 
cyan and green colours, building roof category holds points in yellow, blue and black colours.

Points within each category are arranged in ascending order of elevations.

Figure 5.19 shows the AR of $k$; one means $k$ is accepted, zero otherwise. Figure 5.19 (a) offers overall AR, because of high iteration number, it is not clear to demonstrate switch tendency. Figures 5.19 (b), (c) and (d) extract 5,000 repetitions from the beginning, start of second of computing stage $(150,000$ to 550,000 iterations) and last phase towards end of calculation. It is clearly observable that AR declines steadily during the whole computing process.

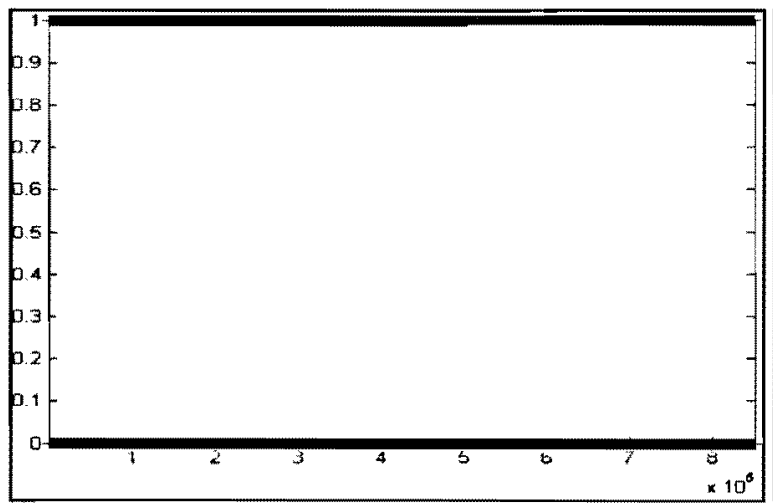

(a)

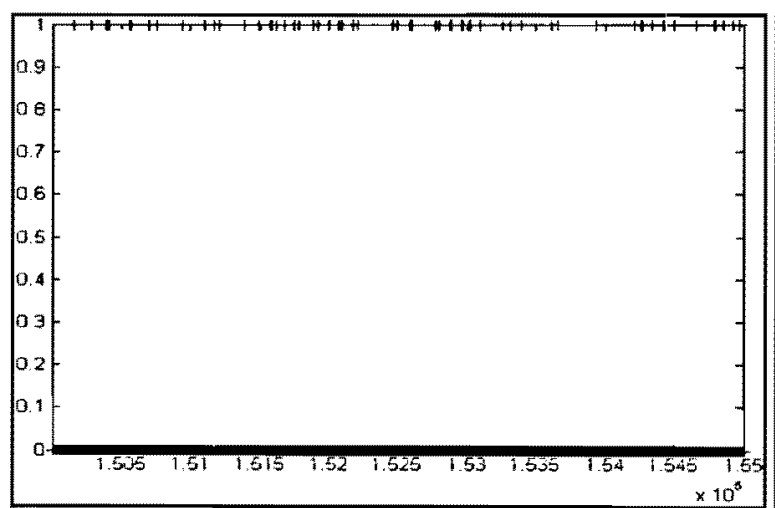

(c)

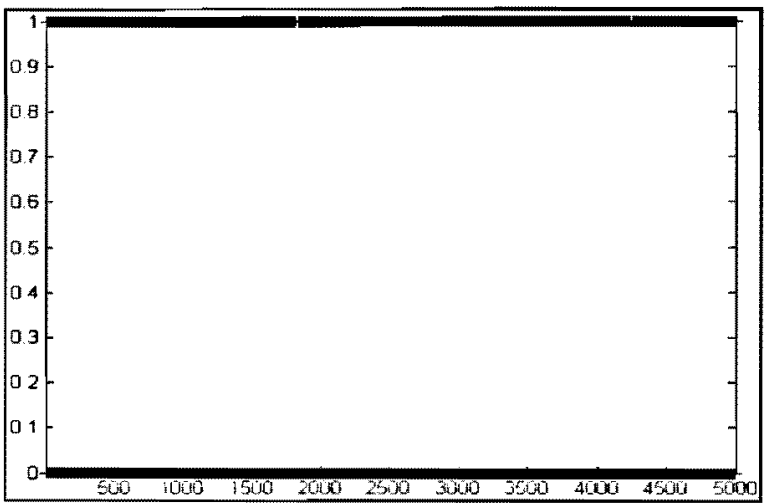

(b)

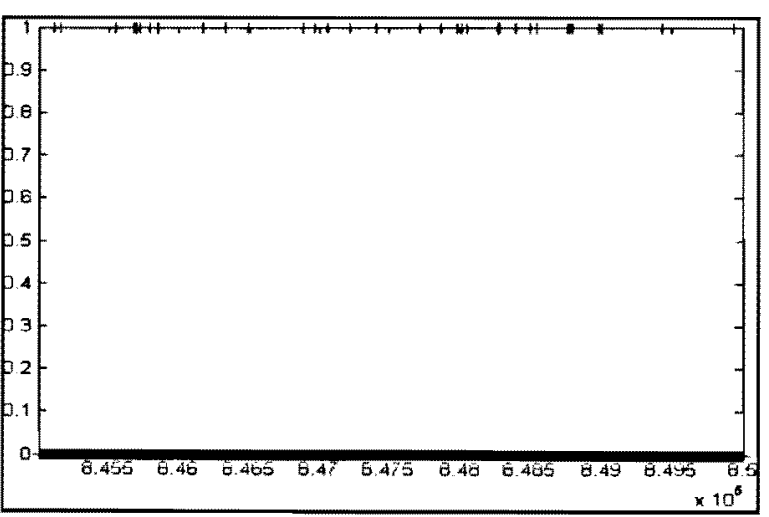

(d)

Figure 5.19 Acceptance rate of $k$ 
Figure 5.20 depicts the alteration of means. With reference to Figure 5.18 (d), Table 5.6 lists point set that each mean belongs to in the geographic distribution sense. The property of each point set is identified in the third row.

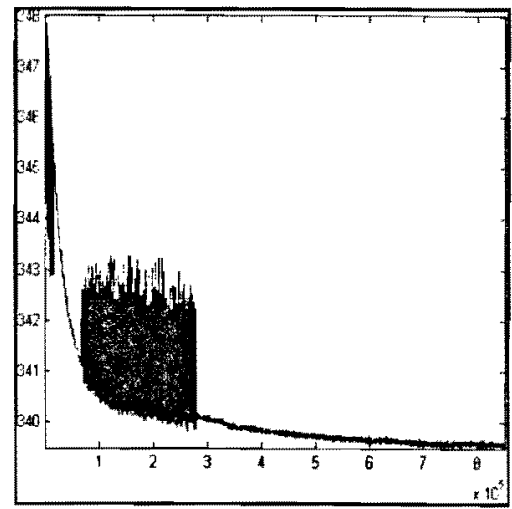

(a)

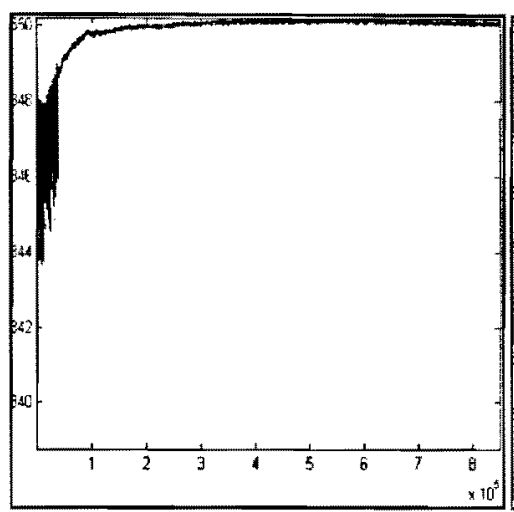

(d)

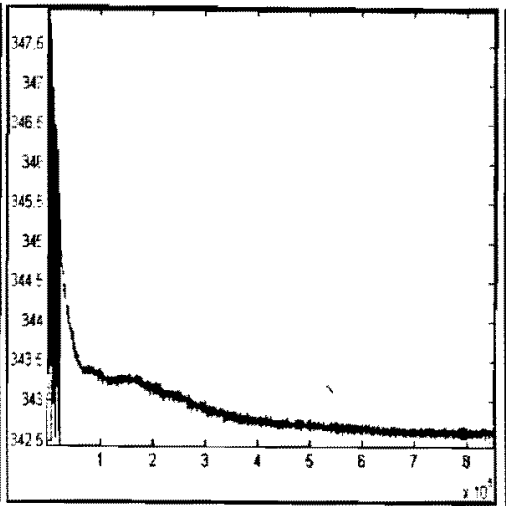

(b)

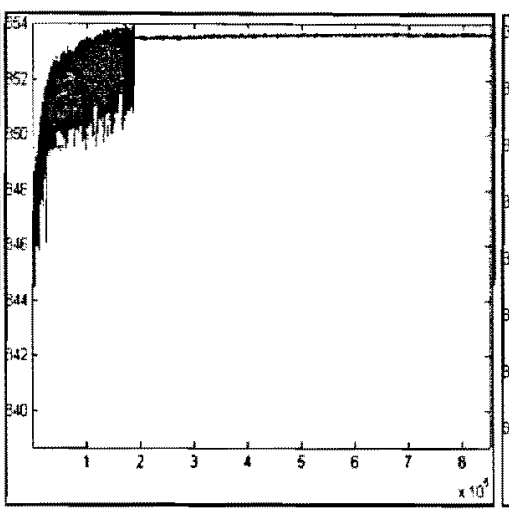

(e)

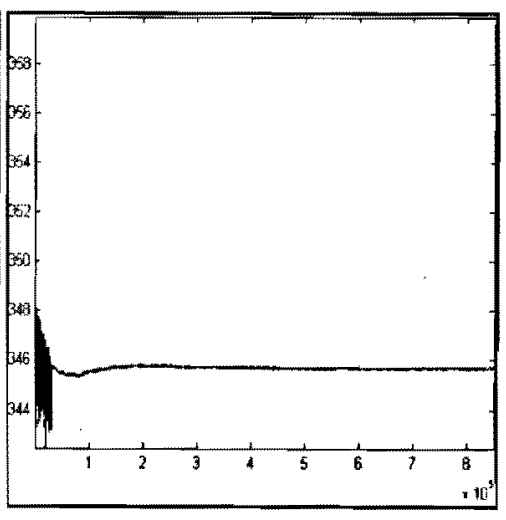

(c)

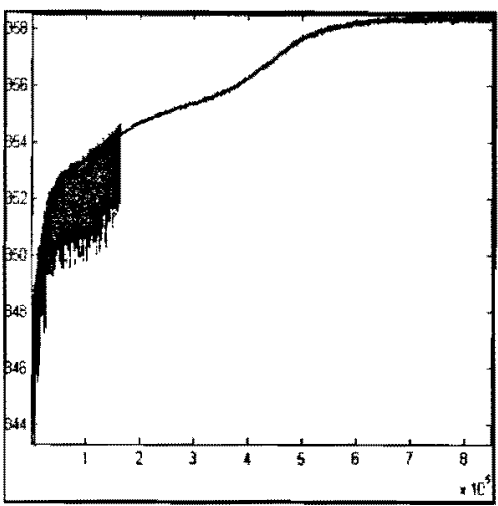

(f)

Figure $5.20 \mu$ Values against iteration

Table 5.6 Means and their colour representations

\begin{tabular}{|l|c|c|c|c|c|c|}
\hline Image name & (a) & (b) & (c) & (d) & (e) & (f) \\
\hline Colour & red & cyan & green & yellow & blue & black \\
\hline Category & ground & ground & ground & building roof & building roof & building roof \\
\hline
\end{tabular}

Due to the range reduction of $\sigma^{2}$ and $\varepsilon^{2}$ and increase of $k$, a couple of changes are noticeable 
in Figure 5.20. First, mean stabilizing times are greatly postponed and in Figure $5.20(f)$, the mean becomes constant after 700,000 iterations. Second, it becomes harder to differentiate means, each mean experiences a period where its value sways heavily, as the calculation progresses, the value stabilizes at certain cycle.

Figure 5.21 shows the AR of $\mu_{I}$ with value changes in Figure 5.20 (a) where one indicates that mean value is accepted and zero otherwise. Figure 5.21 (a) represents comprehensive AR during whole computing cycle. In Figures 5.21 (b), (c) and (d) 2,000 iterations are extracted from the beginning, middle and end parts, respectively to describe changing trends in more detail. At the initial stage, AR is high because of frequent label switch of points and, then it keeps decreasing until the end of computation.

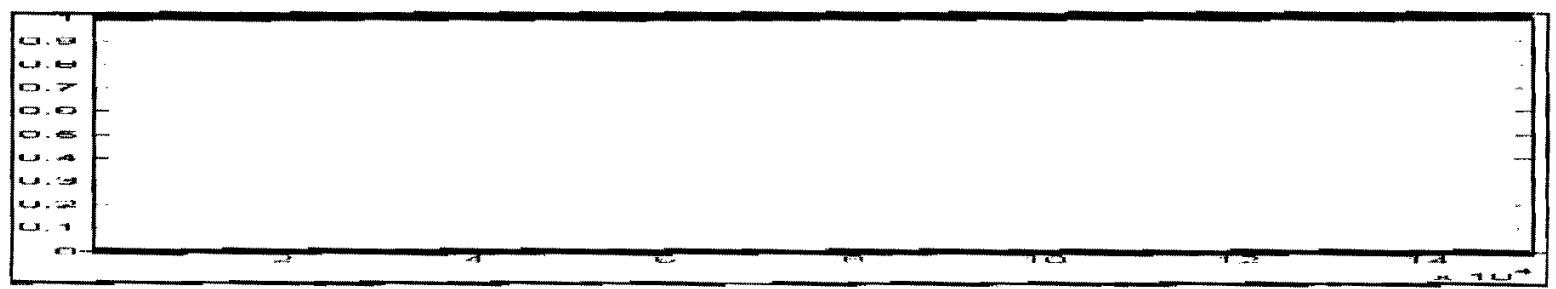

(a)

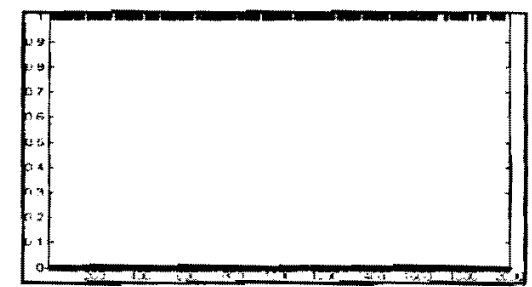

(b)

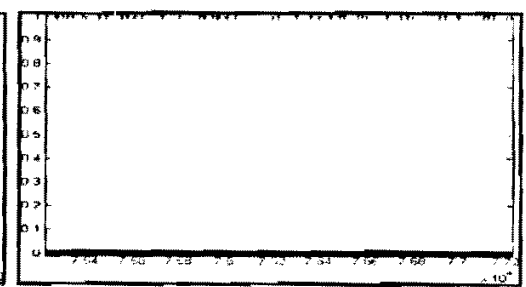

(c)

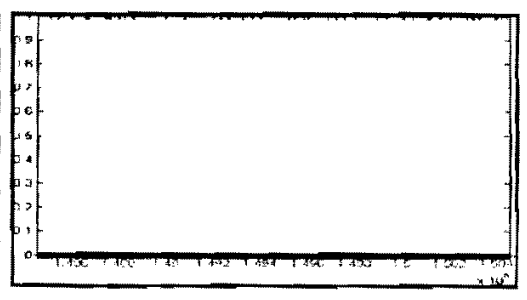

(d)

Figure 5.21 Acceptance rate of $\mu_{I}$

Figure 5.22 pictures the AR of $\mu_{2,}$, whose value change is shown in Figure 5.20 (b), its shift 
trend is similar to $\mu_{1}$.

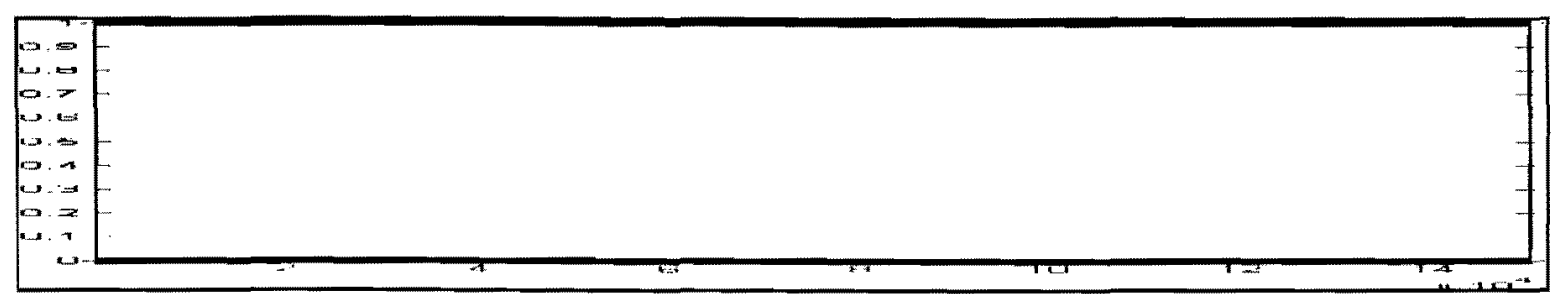

(a)

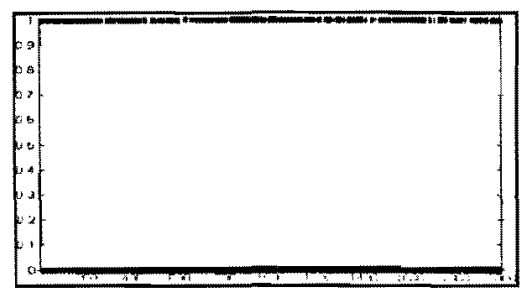

(b)

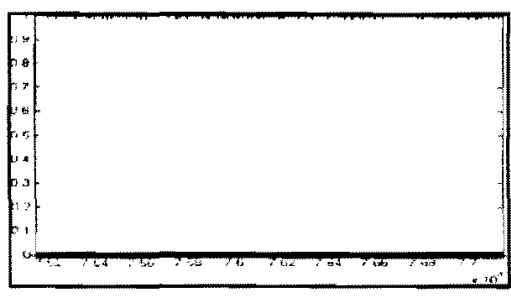

(c)

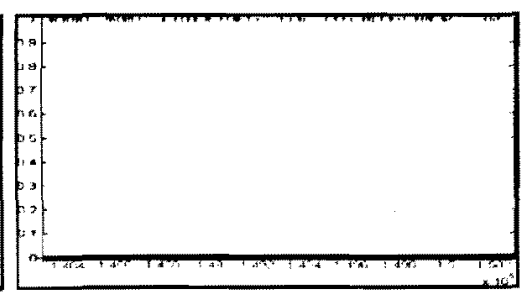

(d)

Figure 5.22 Acceptance rate of $\mu_{2}$

Figure 5.23 indicates AR of $\mu_{3}$, whose value mutation is shown image (c) of Figure 5.20 (c). Its AR follows the same rule as $\mu_{1}$.

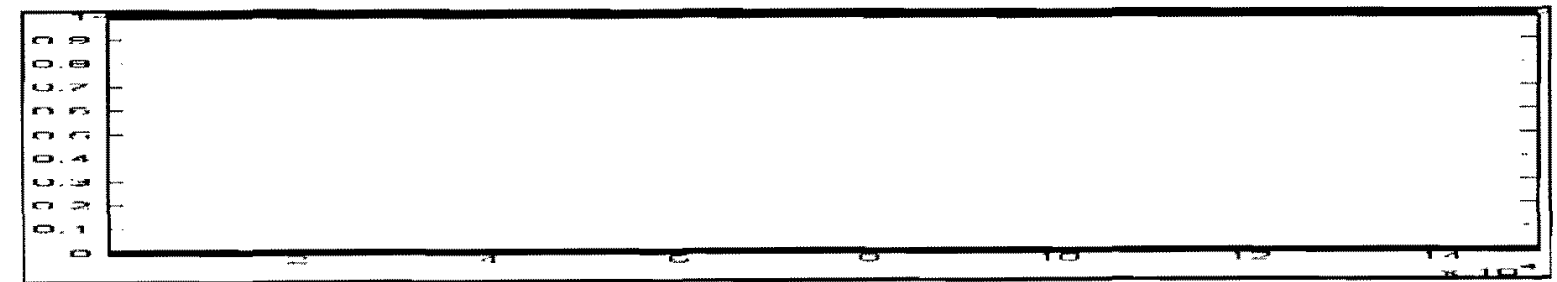

(a)

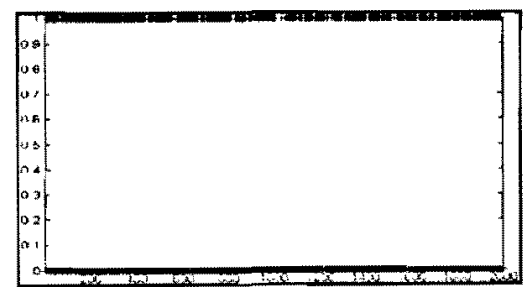

(b)

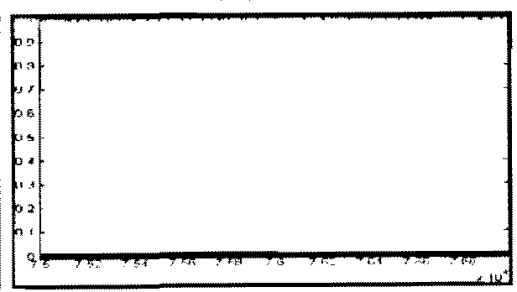

(c)

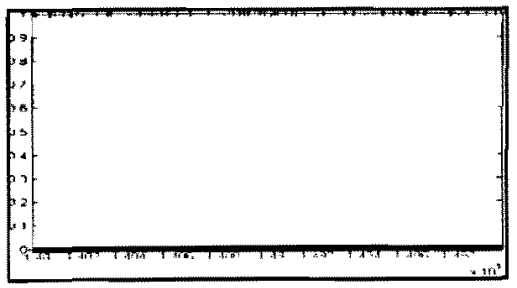

(d)

Figure 5.23 Acceptance rate of $\mu_{3}$

Figure 5.24 depicts AR of $\mu_{4}$, its value change are shown in Figure 5.20 (d), still same order 
as for $\mu_{1}$ governs its alternation tendency.

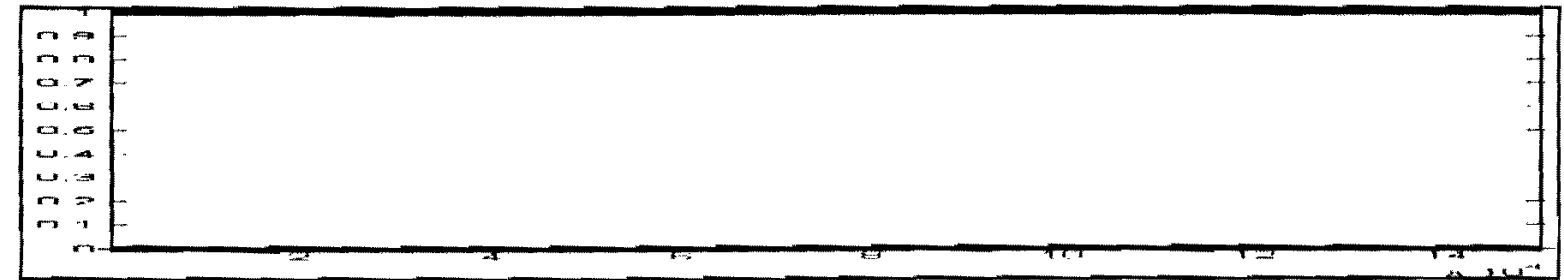

(a)

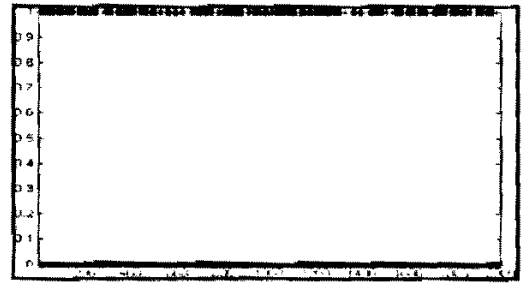

(b)

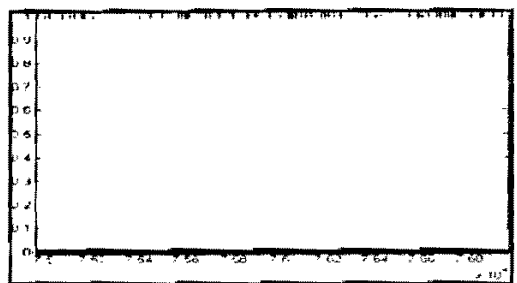

(c)

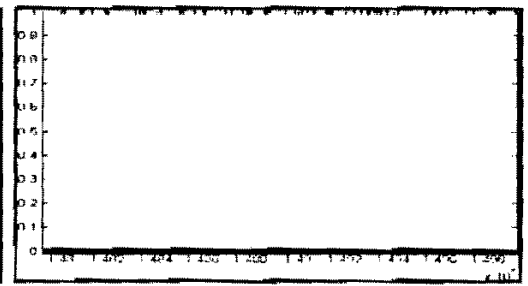

(d)

Figure 5.24 Acceptance rate of $\mu_{4}$

Figure 5.25 shows the $\mathrm{AR}$ of $\mu_{5}$, its value change are displayed in Figure 5.20 (e), its AR experiences single decreasing period, which is identical with AR of $\mu_{I}$.

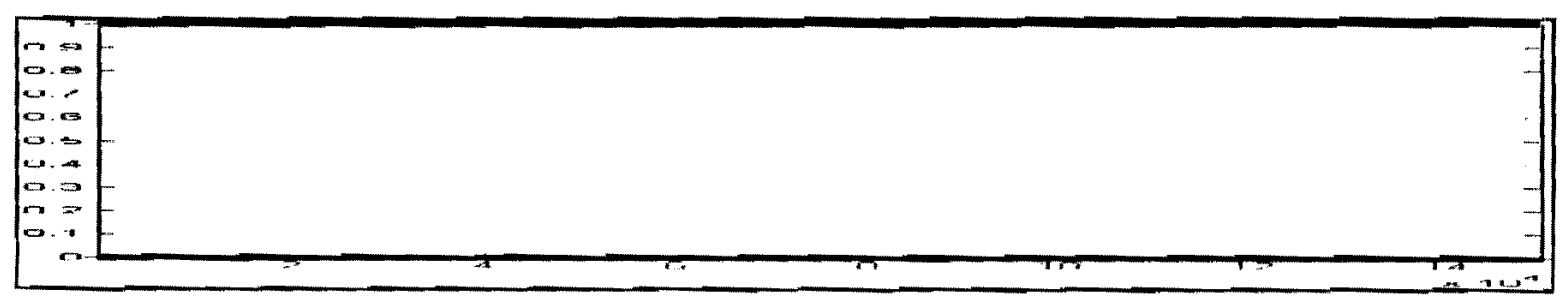

(a)

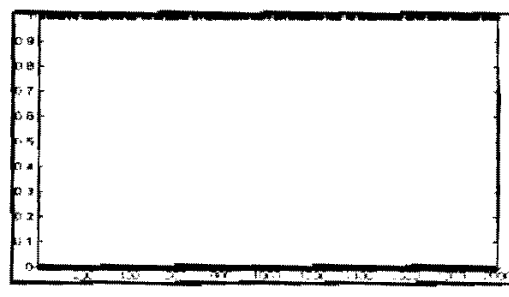

(b)

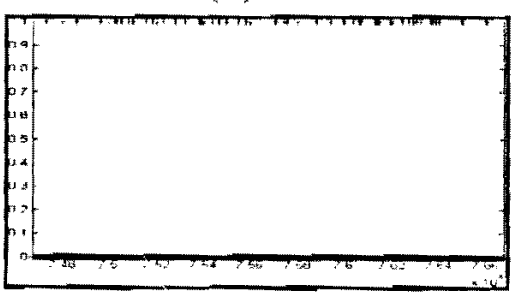

(c)

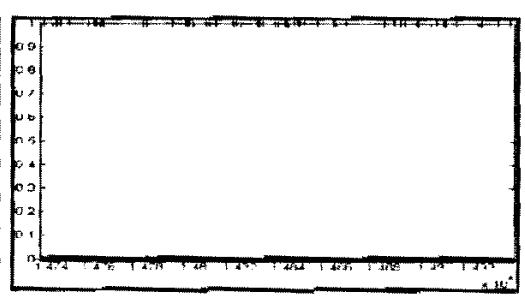

(d)

Figure 5.25 Acceptance rate of $\mu_{5}$

Figure 5.26 displays the $\mathrm{AR}$ of $\mu_{6}$ with its value changes shown in Figure 5.20 (f). Observing from Figure 5.26 (b), (c) and (d) it is obvious that its AR complies with the same rule with $\mu_{1 \text {. }}$ 


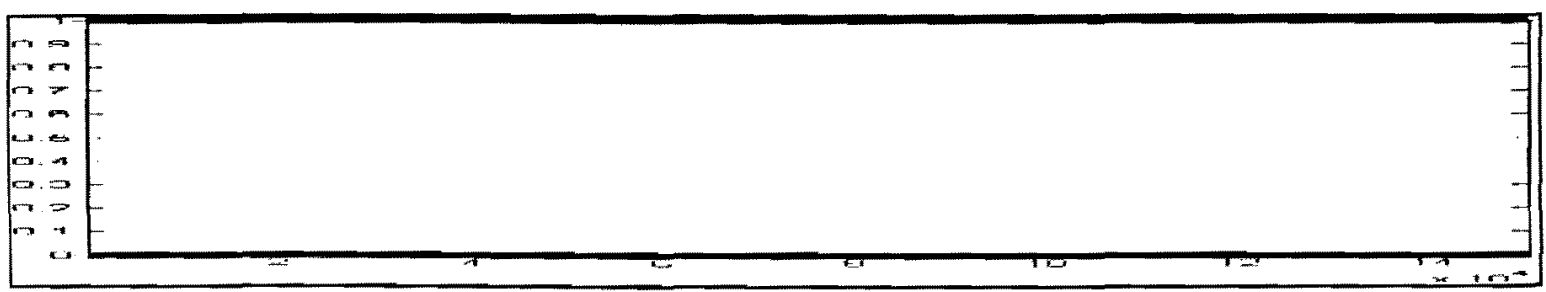

(a)

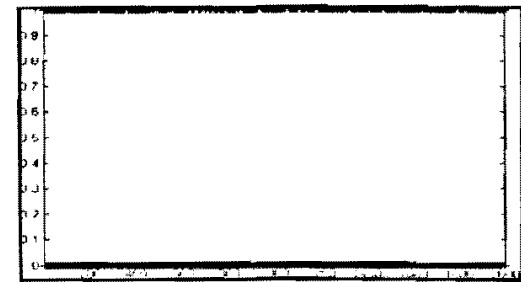

(b)

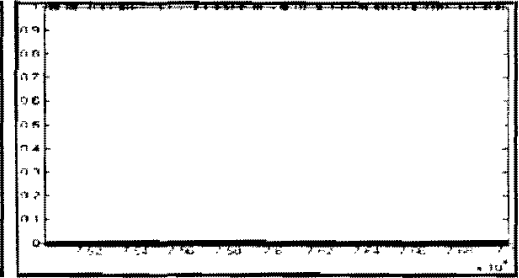

(c)

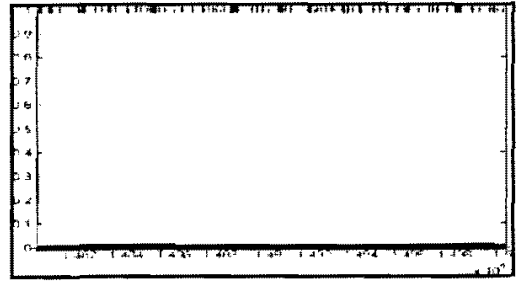

(d)

Figure 5.26 Acceptance rate of $\mu_{6}$

\subsubsection{Filtering}

It is quite common that building roof points are accompanied by HRV points. In the study, both the BFG building and the Optometry building are surrounded by HRV, which can be clearly observed in Figure 5.12 (d) and 5.18 (d). These points are noise and have to be removed. In this section, the Optometry building is chosen to demonstrate the approach.

The Optometry building roof consists of three layers (yellow blue and black colours in Figure 5.27 (a)) with distinct heights. The adjacent area has strong presence of HRV and these regions are square-marked in Figure 5.27 (a). In Figure 5.27 (b) and (c), two building roof layers are obtained which offer a detailed view of HRV points. Points in black colour have no involvement in building roof outline acquisition and will be treated separately in 3D building reconstruction. 


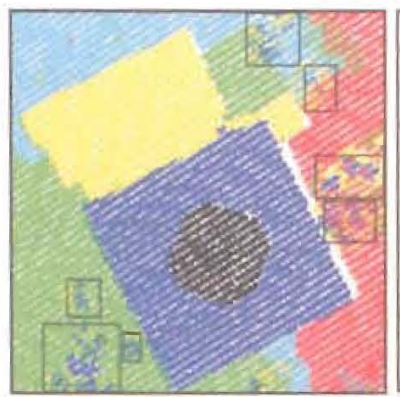

(a)

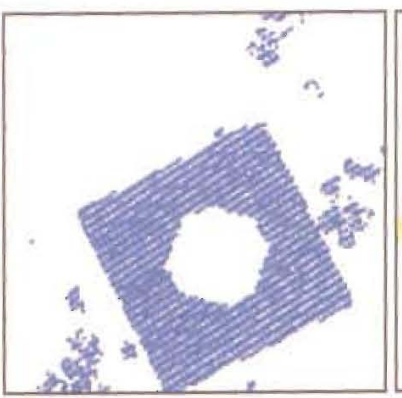

(b)

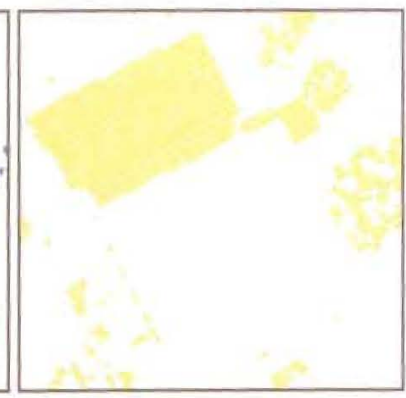

(c)

Figure 5.27 Optometry building and its neighboring HRV

Traditional image filtering approach is borrowed to remove HRV points by applying dilation and erosion filters repeatedly. The process, which consists of two phases, starts by converting points to grayscale image by predefined conversion rules. After the noise is removed, image is converted back to points.

Figure 5.28 demonstrates refining method of building roof points in blue colour. In Figure 5.28 (b), points are transformed to black and white image. A 3-pixel dilation filter is applied to eliminate black pixels within building roof outline with result displayed in Figure 5.28(c). A 11-pixel erosion filter deletes HRV pixels, only building roof pixels are shown in Figure 5.28 (d). By dilating back 11 pixels, Figure 5.28 (e) is achieved. Figure 5.28 (f) exhibits pixel. difference between Figure 5.28 (c) and (e), which holds HRV pixels plus minor building pixels. After HRV pixels are deleted, remaining building pixels are converted back to points, the outcome is shown in Figure $5.28(\mathrm{~g})$. 


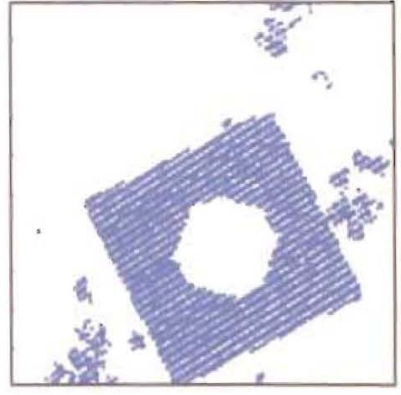

(a)

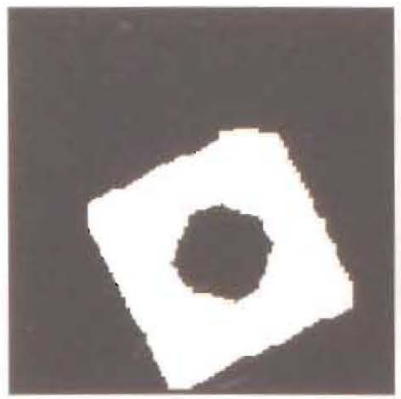

(e)

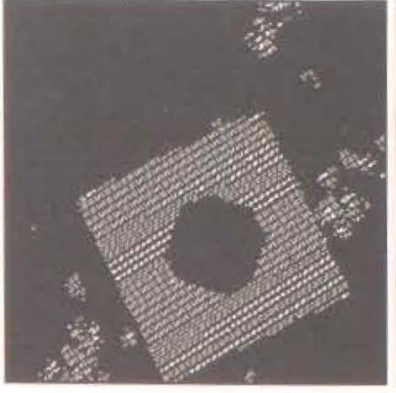

(b)

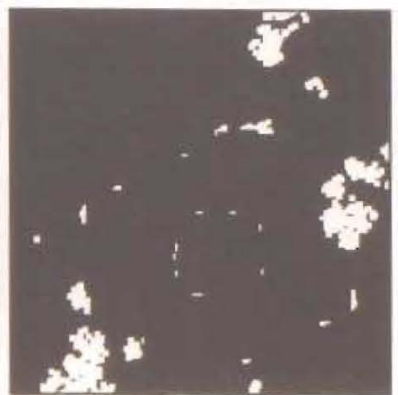

(f)

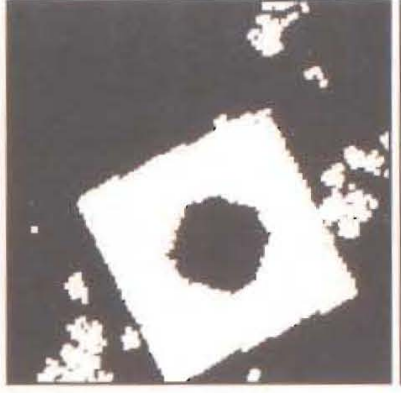

(c)

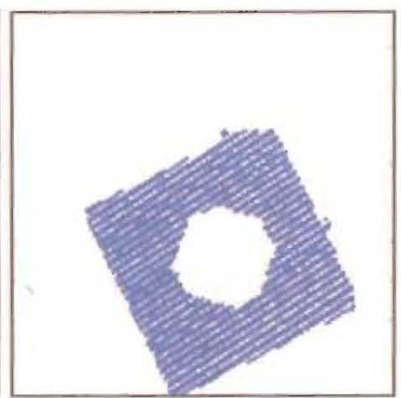

(g)

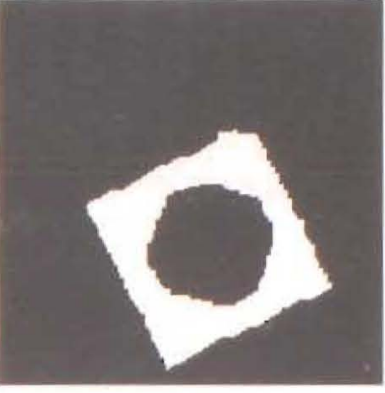

(d)

Figure 5.28 HRV points removal (phase one)

Figure 5.29 demonstrates filtering method of building roof points in yellow colour. Points in blue colour from the previous step are integrated with points in yellow colour as shown in Figure 5.29 (a). Point to image conversion is accomplished, resulting in Figure 5.29 (b). In Figure 5.29 (c) black pixels within building outline are deducted by a 3-pixel dilation filter. Most HRV pixels are eliminated by a 10-pixel erosion filter as indicated in Figure 5.29 (d). Because a small chunk of building area is lost (area in the white polygon), an 18-pixel dilation filter is employed to recover part of missing space indicated by white area covered by polygon in Figure 5.29 (e). An 8-pixel erosion filter is applied to shrink the building area back, the result is exhibited in Figure 5.29 (f). Figure 5.29 (g) shows difference between Figures 5.29 (c) and (f). Image (h) indicates final building points set survived after filtering. 


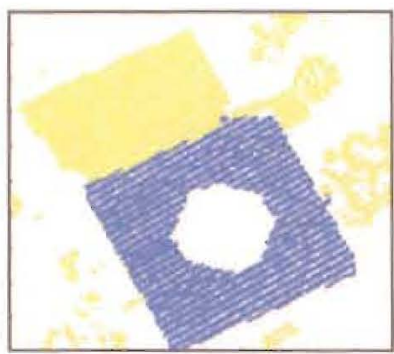

(a)

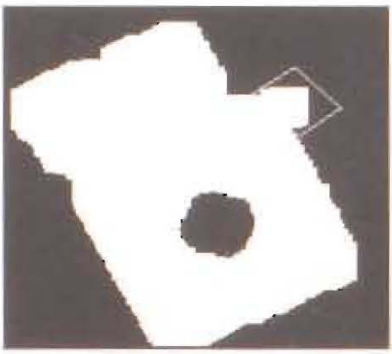

(e)

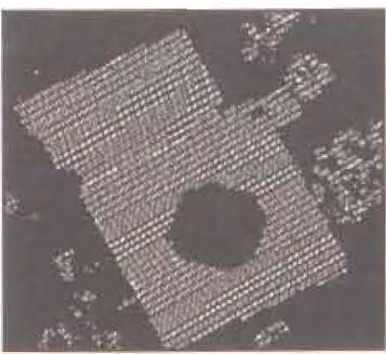

(b)

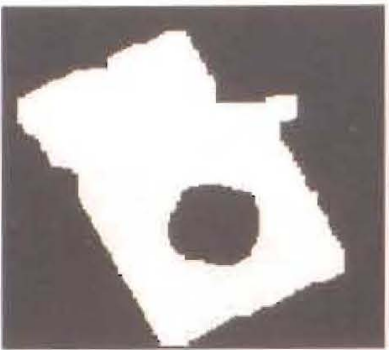

(f)

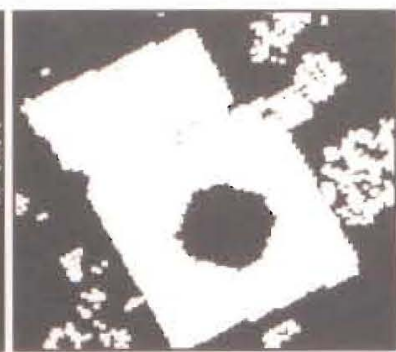

(c)

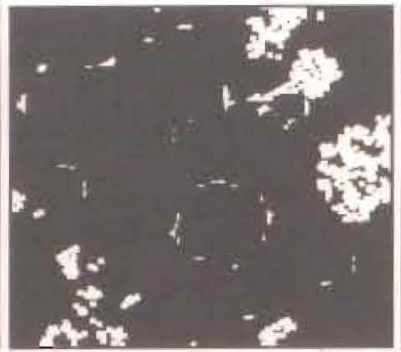

(g)

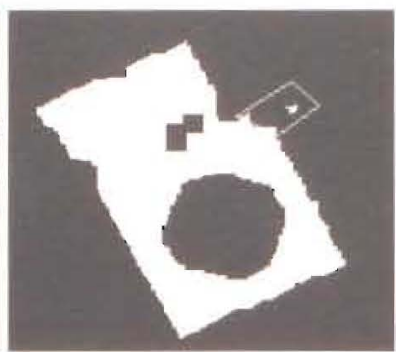

(d)

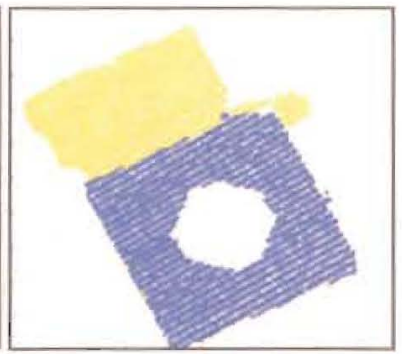

(h)

Figure 5.29 HRV points removal (phase two)

Table 5.7 demonstrates the process of building roof outline points extraction and regularization operations. Images in the first row show the results from the GRFM-MCMC algorithm. In the second row, building point sets are withdrawn together HRV points. By applying the above mentioned filtering technique, HRV points are removed, the remaining building point sets are illustrated in the third row. Roof outline points are further extracted from building points sets through $\mathrm{MCH}$ algorithm with resultants shown in the fourth row. In the last row, roof outline points are regularized by modified hierarchical regularization algorithm. 


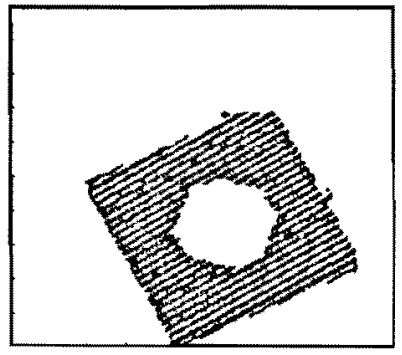

(a)

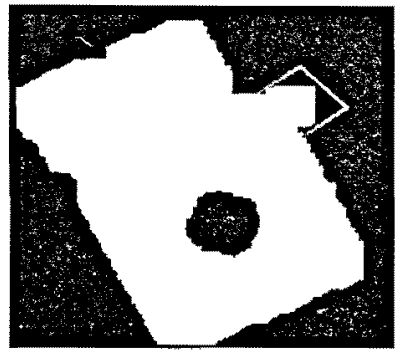

(e)

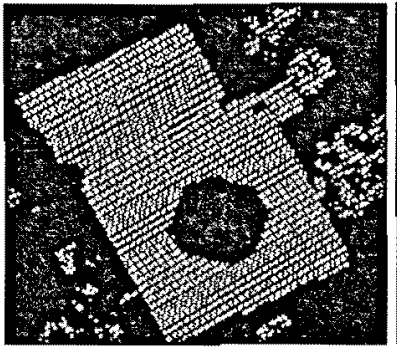

(b)

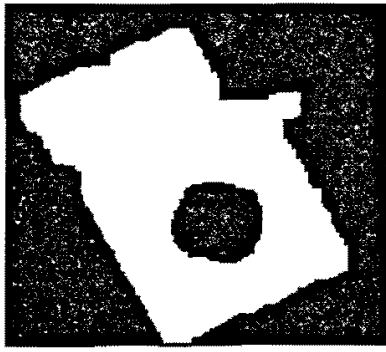

(f)

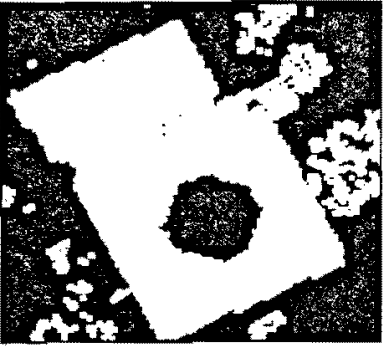

(c)

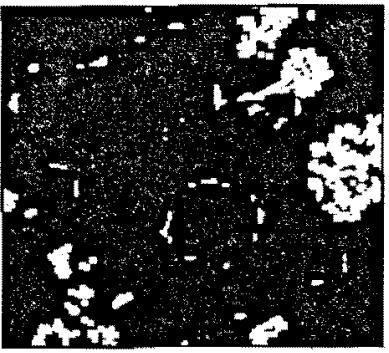

(g)

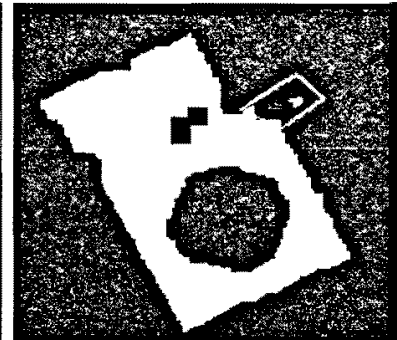

(d)

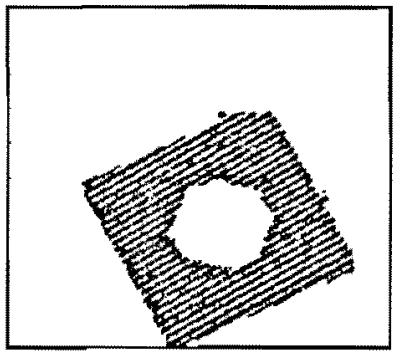

(h)

Figure 5.29 HRV points removal (phase two)

Table 5.7 demonstrates the process of building roof outline points extraction and regularization operations. Images in the first row show the results from the GRFM-MCMC algorithm. In the second row, building point sets are withdrawn together HRV points. By applying the above mentioned filtering technique, HRV points are removed, the remaining building point sets are illustrated in the third row. Roof outline points are further extracted from building points sets through MCH algorithm with resultants shown in the fourth row. In the last row, roof outline points are regularized by modified hierarchical regularization algorithm. 
Table 5.7 Building roof outline extraction and regularization

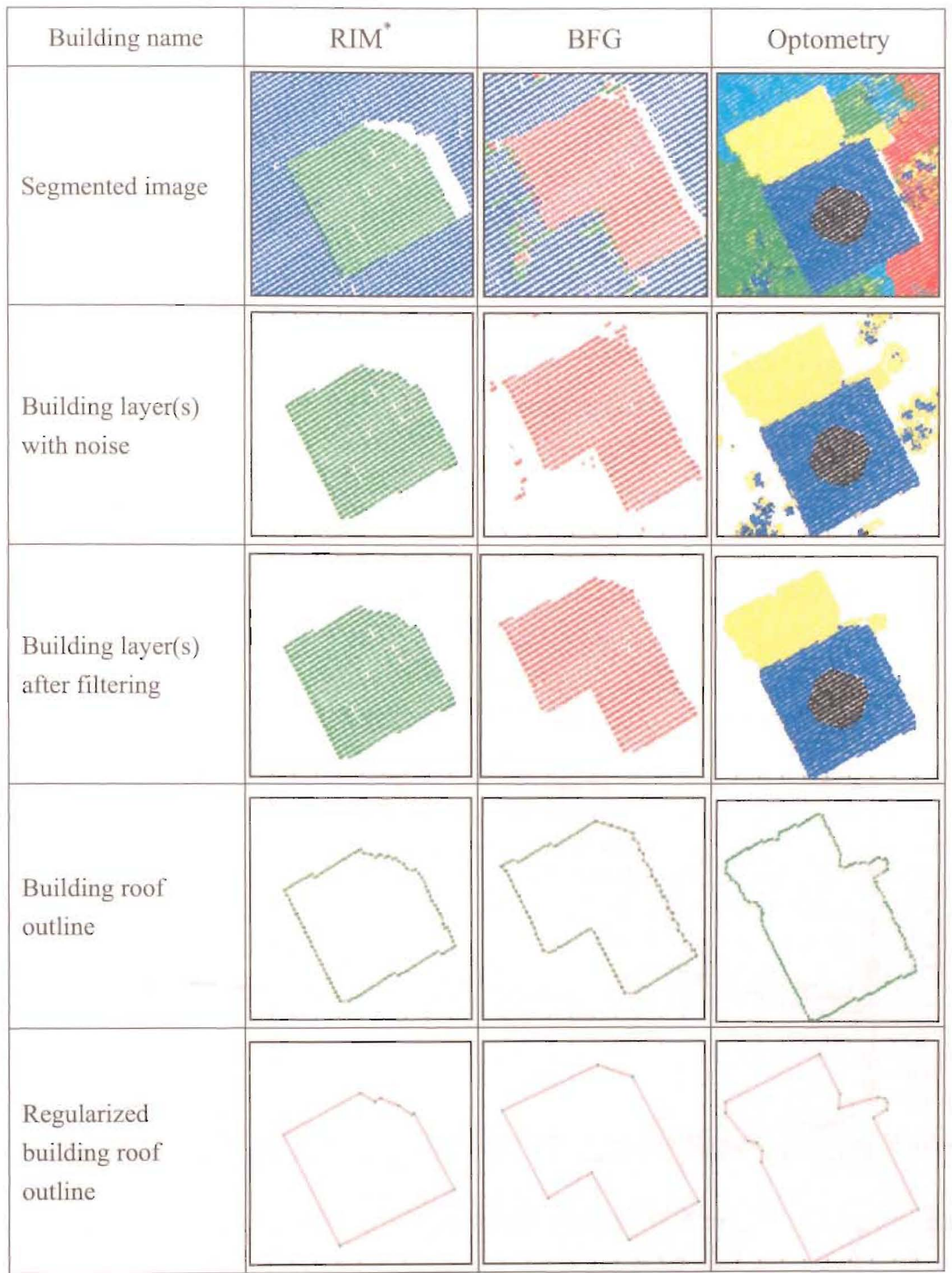

*No filtering process is needed for RIM building, building roof points are identical before and after filtering. 
Table 5.7 Building roof outline extraction and regularization

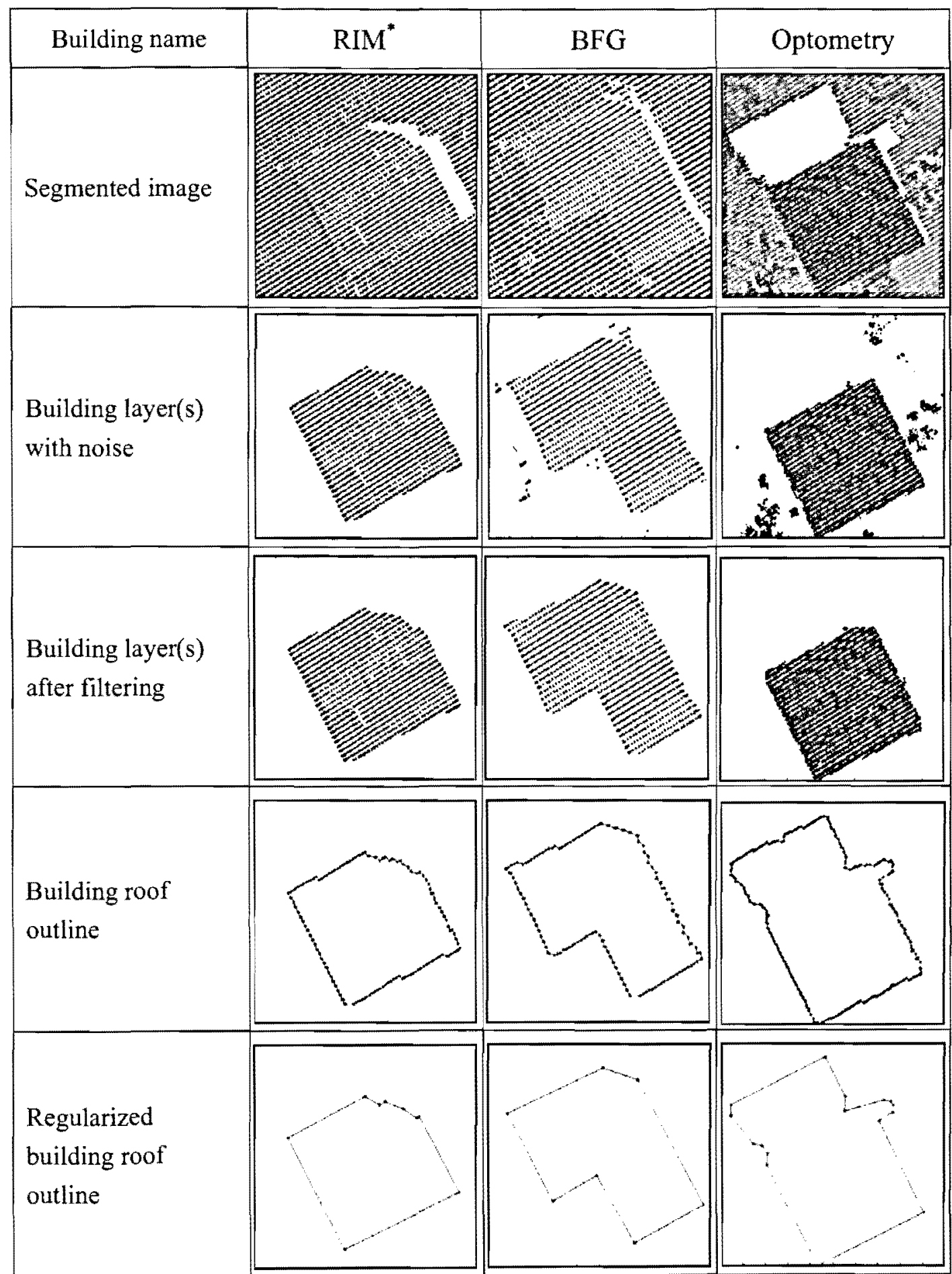

*No filtering process is needed for RIM building, building roof points are identical before and after filtering. 


\subsubsection{D Building Reconstruction in ArcGIS}

After regularization, building edge point sets are ready to be exported to ArcGIS for 3D building reconstruction. The RIM building is taken to demonstrate how to convert geo-referenced point dataset to a polygon shapefile where four steps are involved.

The first step is file re-formatting. Figure 5.30 displays re-formatted border points of the RIM building required by ArcGIS where each row is the spatial reference of one point. The first row and last row must be identical and include a header and end of file mark.

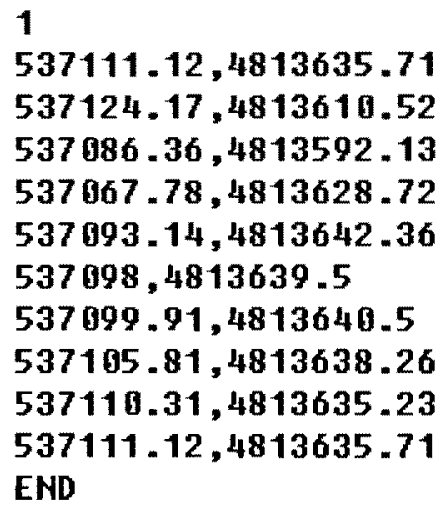

Figure 5.30 File re-formatting of RIM building roof outline points

The second step is creating line coverage. "Generate" function is utilized to convert text file to line coverage file. Figure 5.31 displays the RIM building line coverage file in ArcMap. 


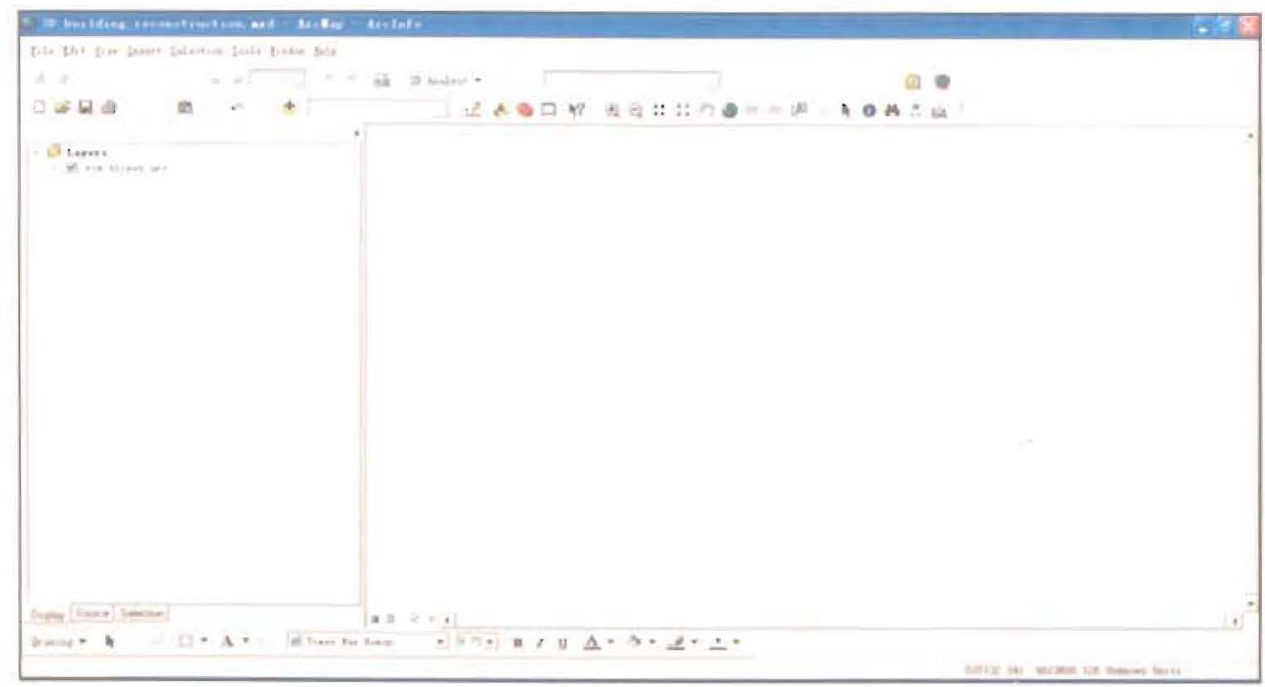

Figure 5.31 RIM building line coverage file in ArcGIS

The third step is polygon shapefile generation. "Feature to Polygon" function is applied to generate polygon shapefile in ArcMap. Figure 5.32 shows the created shapefile.

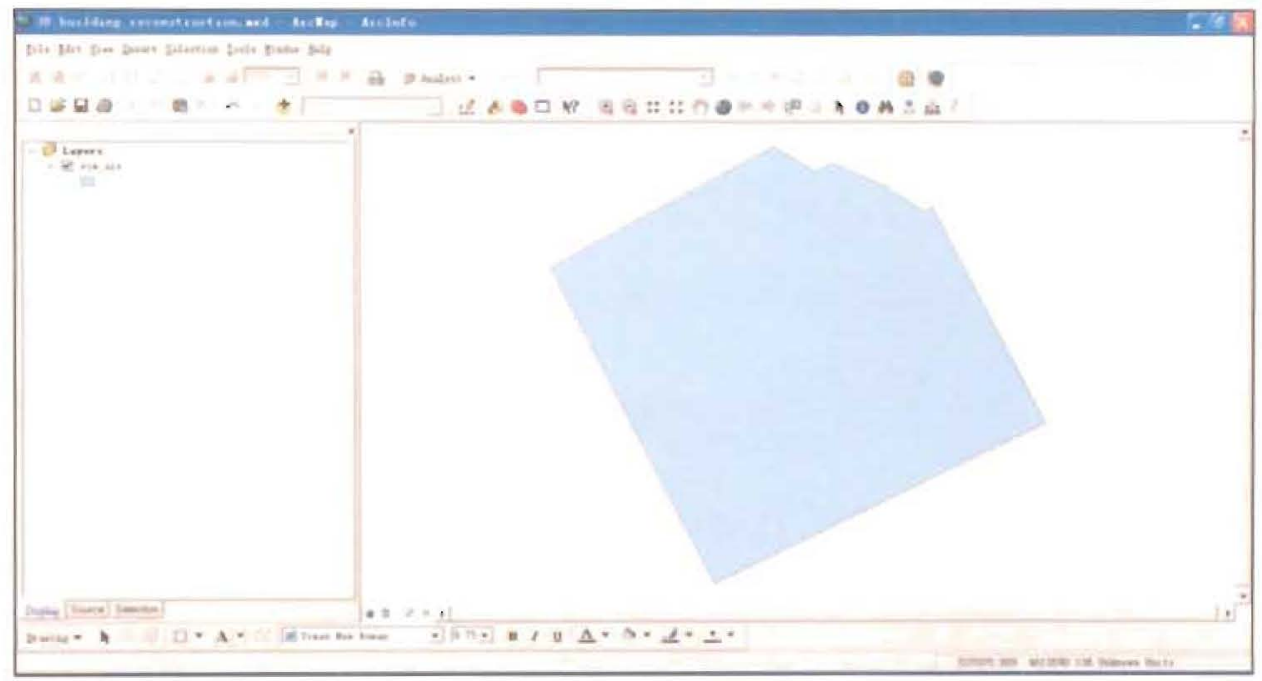

Figure 5.32 RIM building roof shapefile

The last step is assigning the geo-reference system. The "Define Projection" function is employed to attach proper coordinate system to polygon shapefile. As introduced in Chapter 


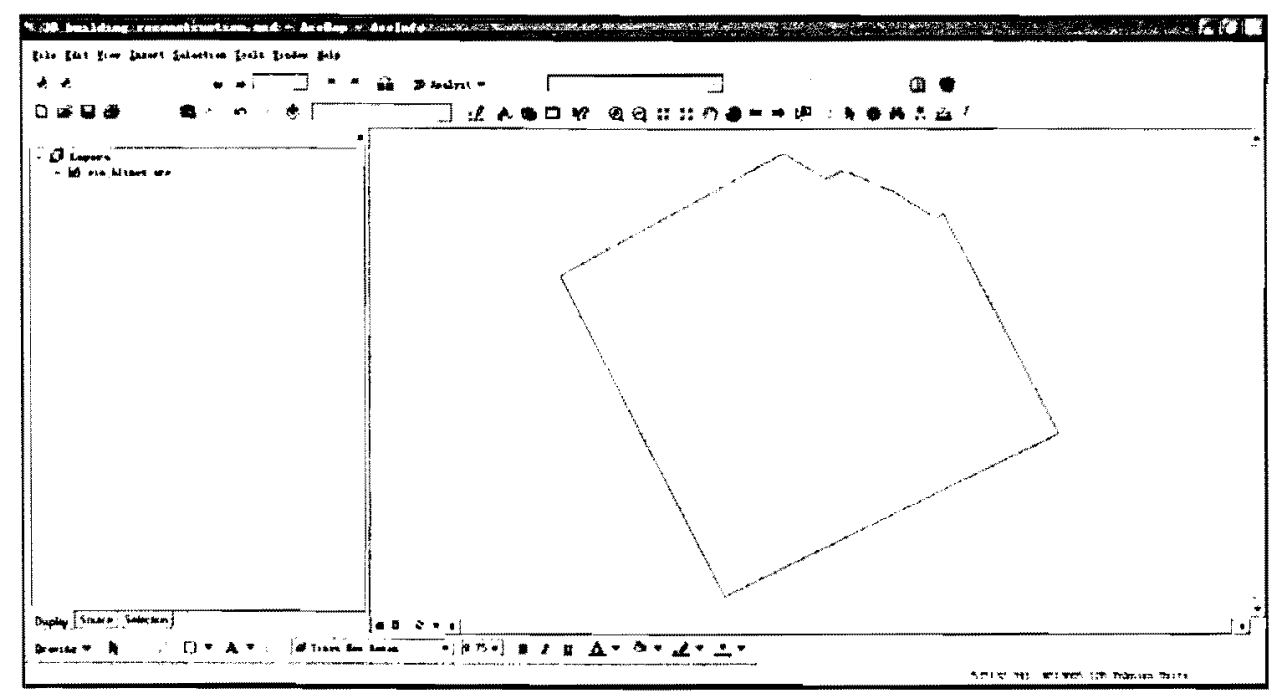

Figure 5.31 RIM building line coverage file in ArcGIS

The third step is polygon shapefile generation. "Feature to Polygon" function is applied to generate polygon shapefile in ArcMap. Figure 5.32 shows the created shapefile.

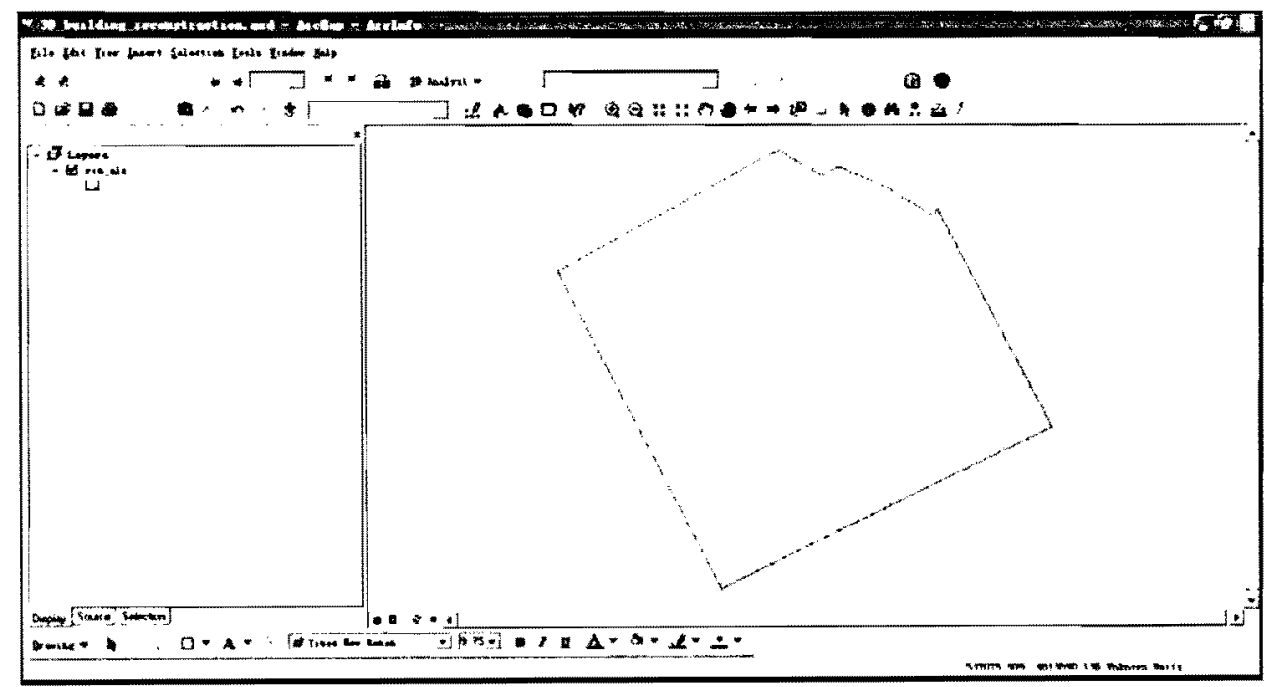

Figure 5.32 RIM building roof shapefile

The last step is assigning the geo-reference system. The "Define Projection" function is employed to attach proper coordinate system to polygon shapefile. As introduced in Chapter 
Three, topographic LiDAR raw data is usually in the UTM system where the UW campus is located in Zone $17 \mathrm{~N}$ and spatial reference system "NAD_1983_UTM_Zone_17N" is selected.

After the above four-step operation, a functional RIM building shapefile is completed, it can not only be used to reconstruct 3D buildings, but also be shared among various geo-databases.

By following identical rules, shapefiles of both BFG and Optometry buildings are generated. Figure 5.33 exhibits the shapesfiles of selected buildings in ArcMap. The BFG building is in blue, the RIM building in olive and the Optometry building in rose.

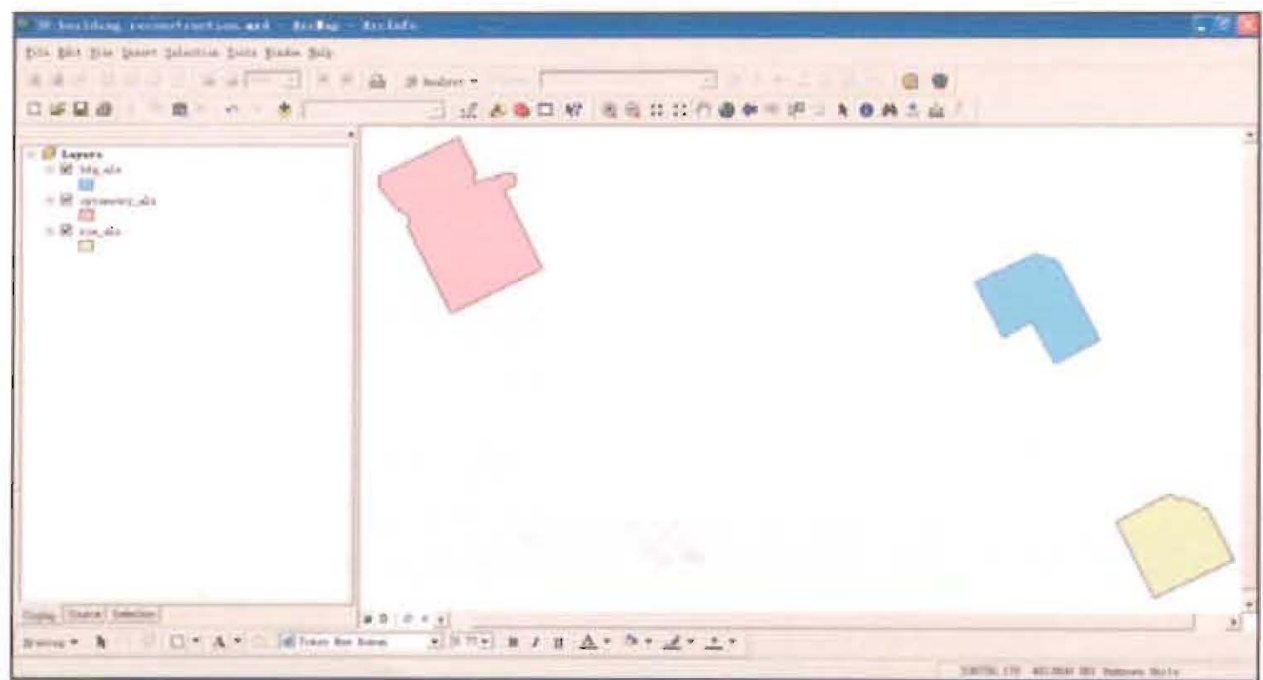

Figure 5.33 Shapefiles of three selected buildings

The height of the building can also be estimated using topographic LiDAR raw data. Elevation 
Three, topographic LiDAR raw data is usually in the UTM system where the UW campus is located in Zone $17 \mathrm{~N}$ and spatial reference system "NAD_1983_UTM_Zone_17N" is selected.

After the above four-step operation, a functional RIM building shapefile is completed, it can not only be used to reconstruct 3D buildings, but also be shared among various geo-databases.

By following identical rules, shapefiles of both BFG and Optometry buildings are generated. Figure 5.33 exhibits the shapesfiles of selected buildings in ArcMap. The BFG building is in blue, the RIM building in olive and the Optometry building in rose.

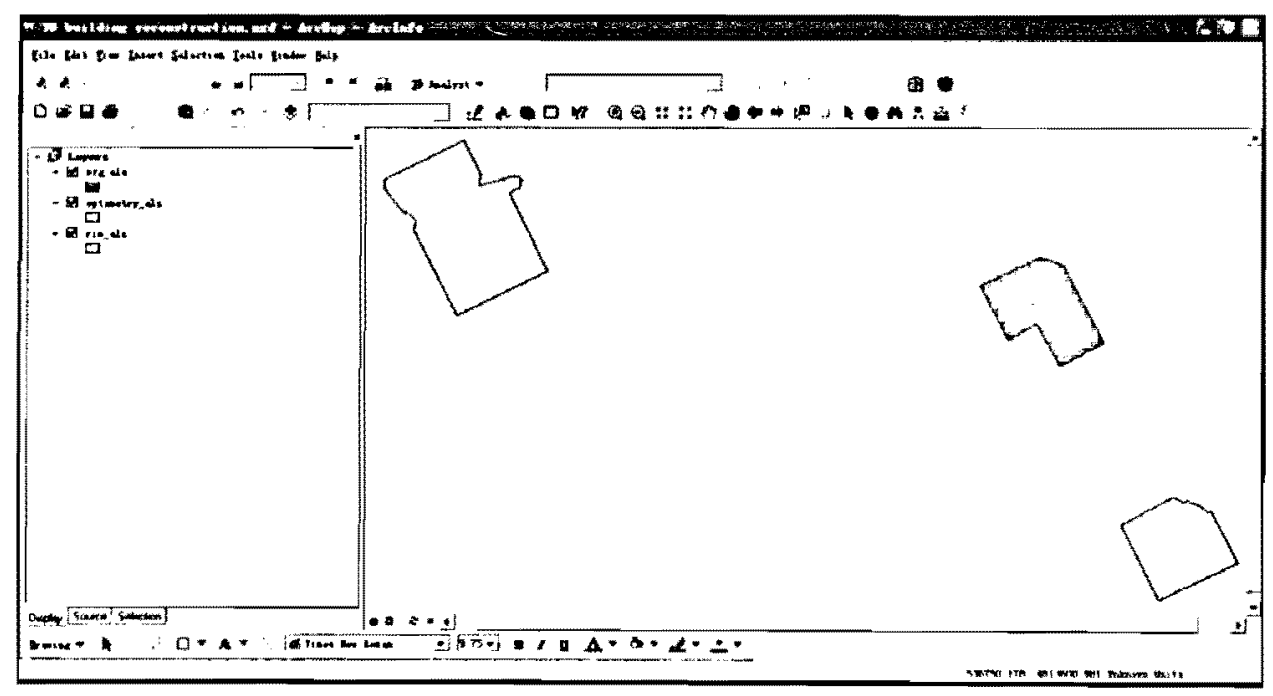

Figure 5.33 Shapefiles of three selected buildings

The height of the building can also be estimated using topographic LiDAR raw data. Elevation 
data for each point is recorded as an absolute value, usually above mean sea level (AMSL). By subtraction the height difference of the building roof layer and ground layer is computable, which can be regarded proximately as the height of building. Table 5.8 shows the calculation process. It is assumed that the ground points, in contrary to HRV points with multiple returns, all come from first return in topographic LiDAR raw data set. In the first row, the building roof points and points with multiple returns are removed and the remaining points are ground points. In the second row, points inside buffer zone with rational width along the building roof outline are picked out as reference points to calculate average height of ground. The width of buffer zones varies from 3 to 5 meters and the reason for buffer zone is that ground area within buffer tends to be flatter than the ground far from building, which can better represent the altitude of ground. In the third and fourth rows, the average altitude of ground and buildings are computed, respectively. In the last row, heights of buildings relative to the ground are figured out by subtraction of values in the third row and the fourth row. Height of buildings will be applied for 3D building reconstruction. 
Table 5.8 Building height estimation

\begin{tabular}{|c|c|c|c|}
\hline Building name & RIM & $\mathrm{BFG}$ & Optometry \\
\hline \multicolumn{4}{|l|}{ Ground points } \\
\hline \multicolumn{4}{|l|}{ Buffer zone } \\
\hline $\begin{array}{l}\text { Altitude of ground } \\
\text { (AMSL) Unit: } m\end{array}$ & 338.08 & 337.88 & 344.01 \\
\hline \multirow{3}{*}{$\begin{array}{l}\text { Altitude of building } \\
\text { (AMSL) Unit: } \mathrm{m}\end{array}$} & \multirow{3}{*}{353.41} & \multirow{3}{*}{346.27} & 350.06 (yellow) \\
\hline & & & 353.65 (blue) \\
\hline & & & 358.35 (black) \\
\hline \multirow{3}{*}{$\begin{array}{l}\text { Height of building } \\
\text { (AGL) Unit: } m\end{array}$} & \multirow{3}{*}{15.33} & \multirow{3}{*}{8.39} & 6.05 (yellow) \\
\hline & & & 9.64 (blue) \\
\hline & & & 14.34 (black) \\
\hline
\end{tabular}

*Optometry building has three parts with different heights.

Based on the elevation data of each building listed in Table 5.8 and shapefiles created before, 3D buildings reconstruction is achievable. Optometry building has three levels of roofs with different altitude, in order to mimic this property in 3D view, its polygon file is split into two parts and another shapefile created from points in black colour is added. A 3D view of building is accomplished in ArcScene. Figure 5.34 provides 3D view of sample buildings, Figure 5.34 (a) is viewed from the North West and Figure 5.34 (b) is from the East. 


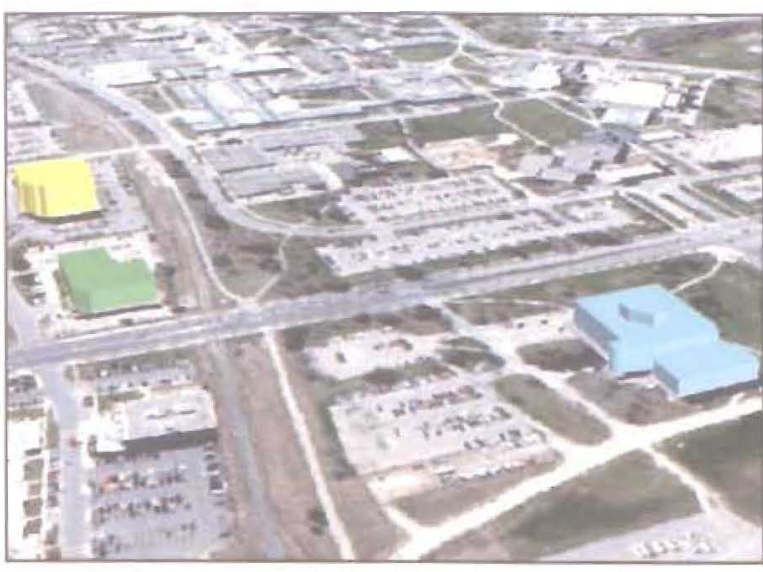

(a)

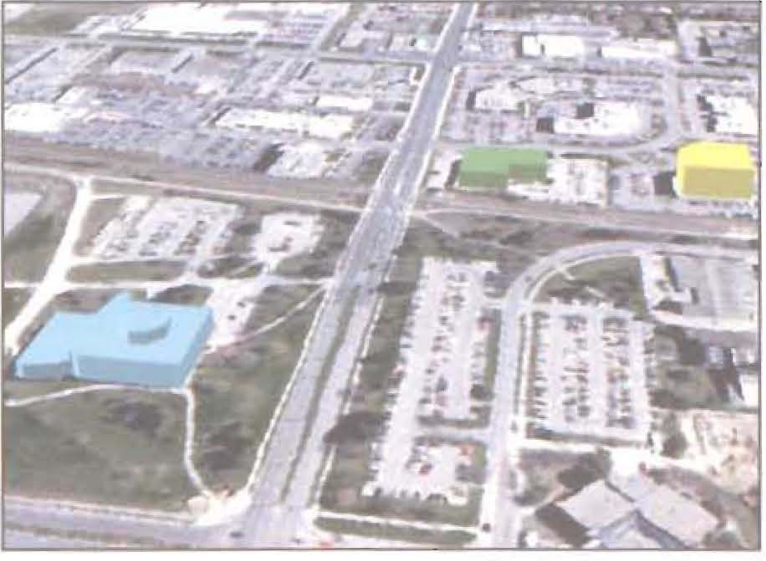

(b)

Figure 5.34 3D view of selected buildings

Figure 5.35 offers a comprehensive 3D view of the UW campus. The building shapefiles are acquired from map library while their height information is from planning office.

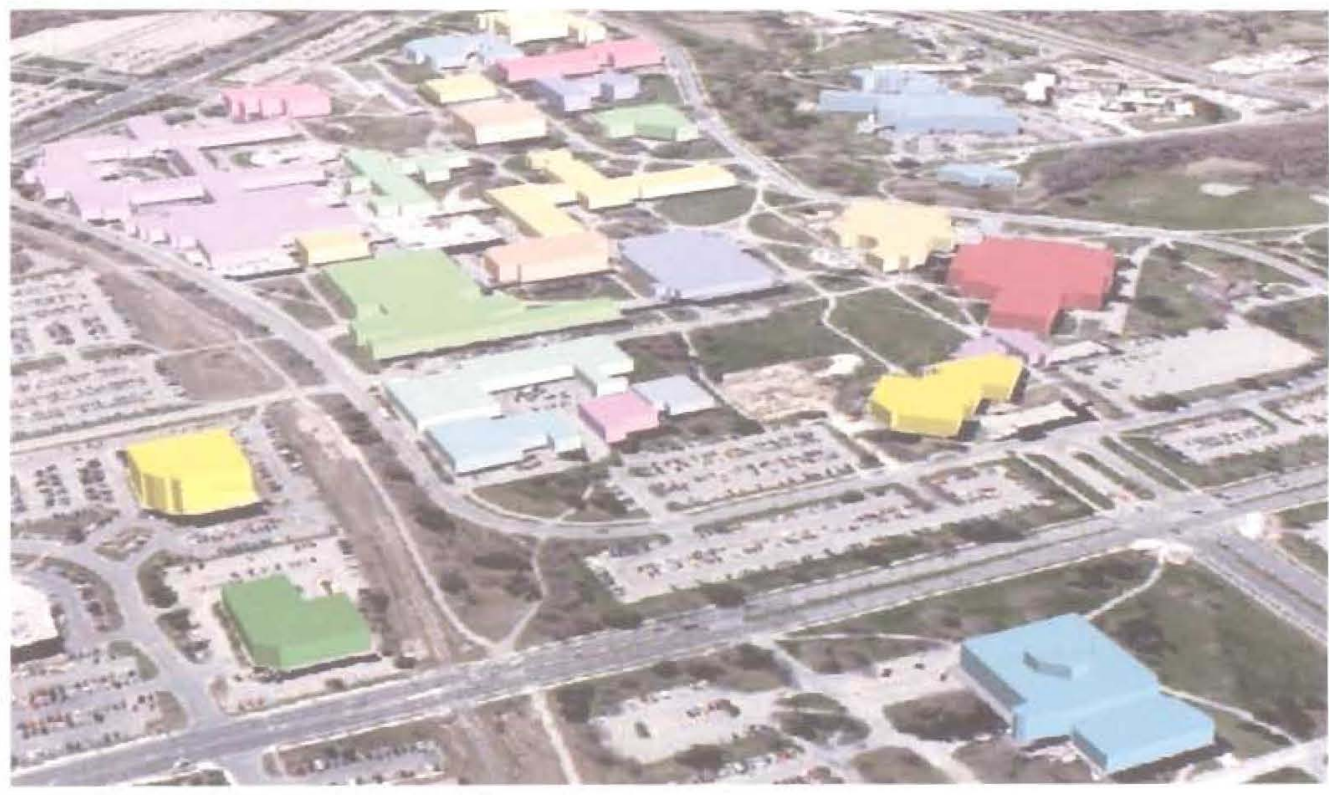

Figure 5.35 3D view of UW campus 


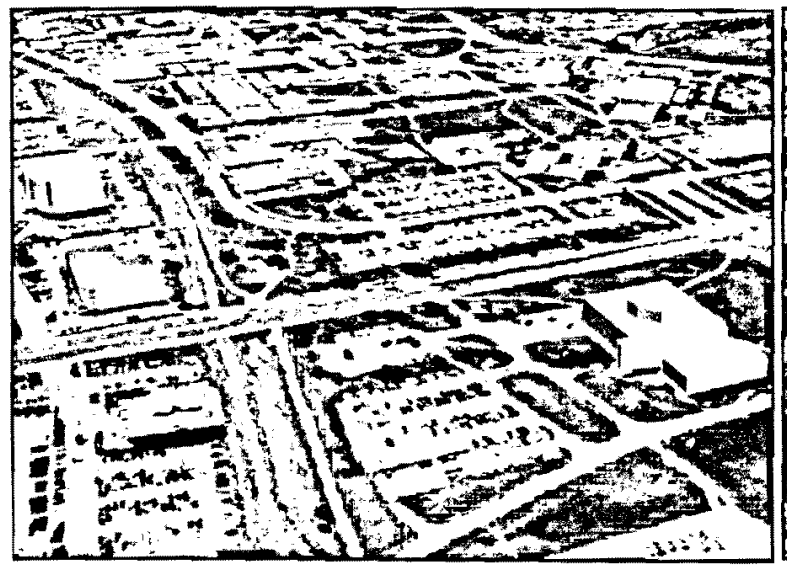

(a)

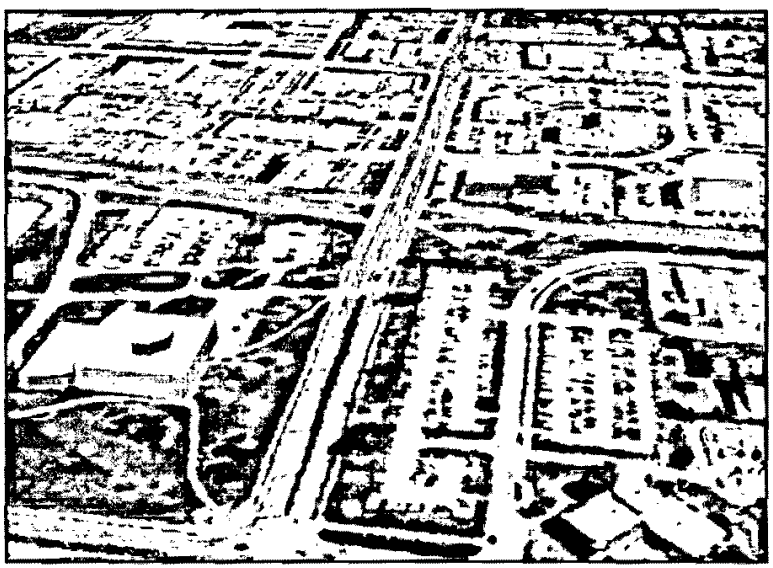

(b)

Figure 5.34 3D view of selected buildings

Figure 5.35 offers a comprehensive 3D view of the UW campus. The building shapefiles are acquired from map library while their height information is from planning office.

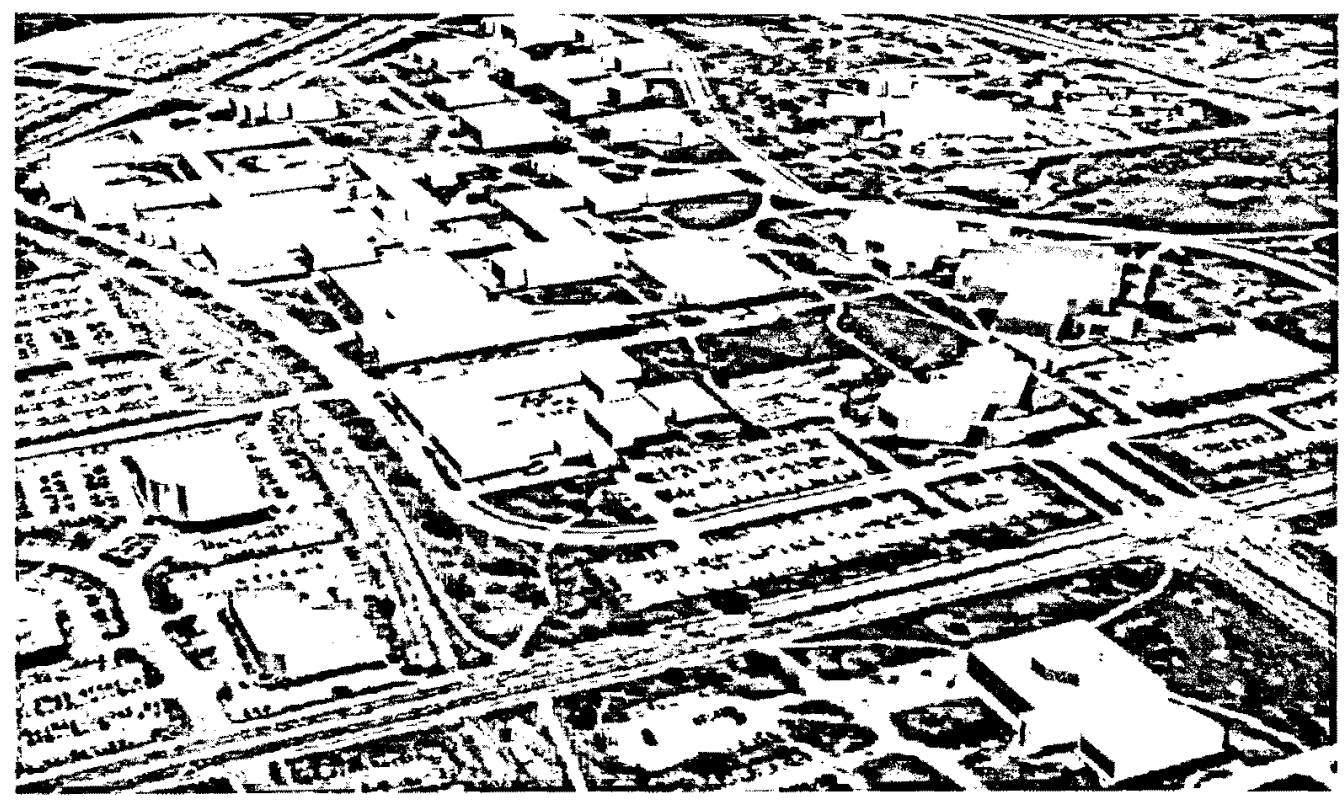

Figure 5.35 3D view of UW campus 


\subsection{Accuracy Evaluation}

In this study, three tools in descriptive statistics, namely overall accuracy (OA), commission error (CE) and omission error (OE) are introduced for accuracy estimation. Measurement of surface area covered by polygon shapefile is used for computation while standard shapefiles from UW map library are imported as a reference. The inspection is achieved in an ArcGIS environment based on individual building polygon. The formula and brief explanation of each tool is provided as follows:

1. Overall Accuracy $(\mathrm{OA})$

As the name suggests, OA tests how well the building shape is recovered according the performance of the algorithm.

$$
O A=\frac{A_{r e f}}{A_{a l s}}
$$

$A_{r e f}$ is the area of reference building polygon, $A_{\text {als }}$ is the area of building polygon from topographic LiDAR raw data.

2. Commission Error (CE)

CE indicates how much area of the building polygon is recovered where it should not. The designer of the algorithm is more concerned about $\mathrm{CE}$, because it tells the correct interpretation rate building shape.

$$
C E=\frac{A_{a l s}-\left(A_{a l s} \cup A_{r e f}\right)}{A_{a l s}}
$$




\section{Omission Error (OE)}

$\mathrm{OE}$ implies how much area of the building polygon fails to be recovered where it should be found. The user takes more care of the value, because it tells the percentage of the building shape that is correctly restored.

$$
O E=\frac{A_{r e f}-\left(A_{a l s} \cup A_{r e f}\right)}{A_{r e f}}
$$

Figure 5.36 exhibits overlay effect of shapefiles. Standard shapefiles are in solid red line, generated ones are in yellow colour. In all three images it is noticeable that some area controversies exist between two versions of the shapefiles in each building, by computing area differences, the accuracy rate of proposed algorithm can be estimated.

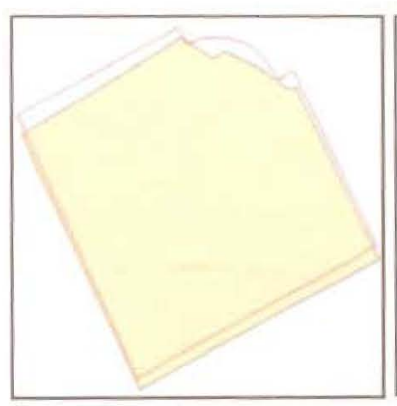

(a)

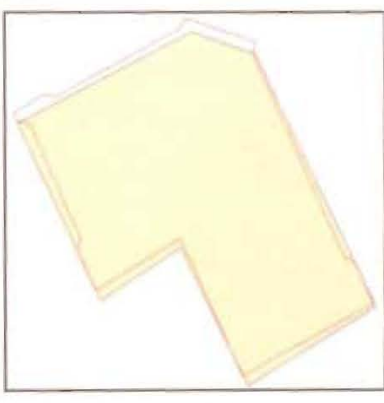

(b)

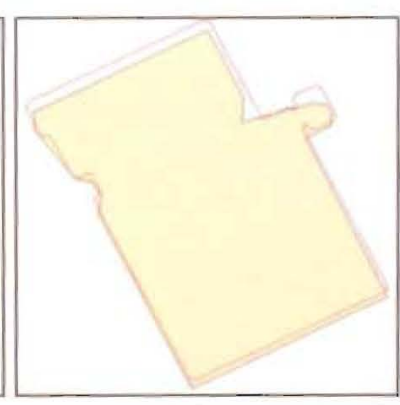

(c)

Figure 5.36 Overlay of shapefiles

Table 5.9 lists the area differences of three buildings in both coloured shapefiles and numbers.

Given two versions of shapefiles in 


\section{Omission Error (OE)}

OE implies how much arca of the building polygon fails to be recovered where it should be found. The user takes more care of the value, because it tells the percentage of the building shape that is correctly restored.

$$
O E=\frac{A_{r e f}-\left(A_{\text {cls }} \cup A_{\text {ref }}\right)}{A_{\text {ref }}}
$$

Figure 5.36 exhibits overlay effect of shapefiles. Standard shapefiles are in solid red line, generated ones are in yellow colour. In all three images it is noticeable that some area controversies exist between two versions of the shapefiles in each building, by computing area differences, the accuracy rate of proposed algorithm can be estimated.

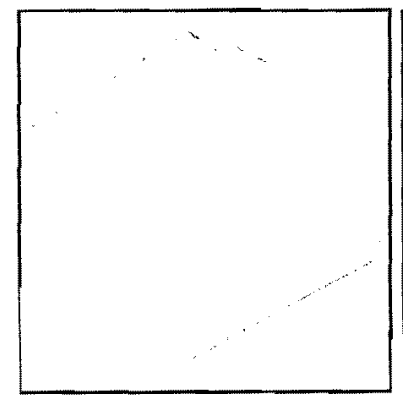

(a)

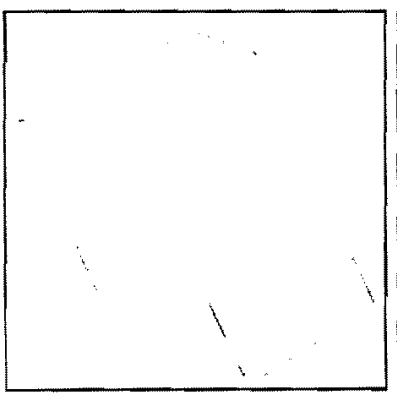

(b)

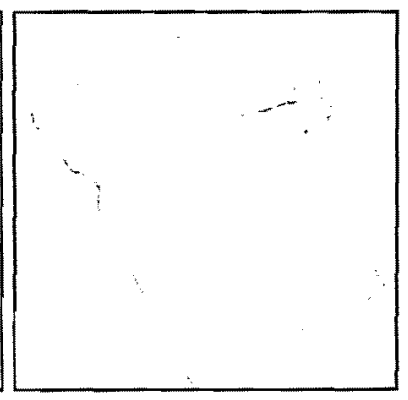

(c)

Figure 5.36 Overlay of shapefiles

Table 5.9 lists the area differences of three buildings in both coloured shapefiles and numbers.

Given two versions of shapefiles in 
Figure 5.36, new shapefiles which carry the union area coverage of both shapefiles are created except that they are divided into three sections. The area in rose colour is the area that both shapefiles agree, the area in blue colour is the area in the standard shapefile, but absent from generated shapefile, the area in green colour is the area present in generated shapefile, but missing from standard shapefile. Coloured shapefiles are presented in the second column of Table 5.9. In the third column, the measurements of individual coloured areas are calculated through geometry computing function in ArcGIS.

Table 5.9 Shapefiles and their area coverage

\begin{tabular}{|c|c|c|}
\hline Building names & Union of shapefiles & Area by colour $\left(\mathrm{m}^{2}\right)$ \\
\hline \multirow[t]{3}{*}{ RIM } & & $\square \quad 115.80$ \\
\hline & & $\square \quad 1568.68$ \\
\hline & & $\square \quad 95.25$ \\
\hline \multirow[t]{3}{*}{$\mathrm{BFG}$} & & $\square \quad 63.69$ \\
\hline & & $\square \quad 1607.48$ \\
\hline & & $\square \quad 118.48$ \\
\hline \multirow[t]{3}{*}{ Optometry } & & $\square \quad 232.82$ \\
\hline & & $\square \quad 3459.07$ \\
\hline & & $\square \quad 122.32$ \\
\hline
\end{tabular}


Figure 5.36, new shapefiles which carry the union area coverage of both shapefiles are created except that they are divided into three sections. The area in rose colour is the area that both shapefiles agree, the area in blue colour is the area in the standard shapefile, but absent from generated shapefile, the area in green colour is the area present in generated shapefile, but missing from standard shapefile. Coloured shapefiles are presented in the second column of Table 5.9. In the third column, the measurements of individual coloured areas are calculated through geometry computing function in ArcGIS.

Table 5.9 Shapefiles and their area coverage

\begin{tabular}{|c|c|c|}
\hline Building names & Union of shapefiles & Area by colour $\left(\mathrm{m}^{2}\right)$ \\
\hline \multirow[t]{3}{*}{ RIM } & & ㅁ 115.80 \\
\hline & & ㅁ 1568.68 \\
\hline & & $\square 95.25$ \\
\hline \multirow[t]{3}{*}{$\mathrm{BFG}$} & & ㅁ 63.69 \\
\hline & & ㅁ 1607.48 \\
\hline & & ㅁ 118.48 \\
\hline \multirow[t]{3}{*}{ Optometry } & & ㅁ 232.82 \\
\hline & & ㅁ 3459.07 \\
\hline & & ㅁ 122.32 \\
\hline
\end{tabular}


In Table 5.10, $\mathrm{OA}, \mathrm{CE}$ and $\mathrm{OE}$ of each building are calculated based on the data in Table 5.9, where the averages of each error are listed in the last row as well. OA ranges from almost $97 \%$ to $103 \%$ with an average around $100.38 \%$. Regarding CE, an average of $5.34 \%$ of the area is mistakenly included the created shapefiles. On the other hand, $5.66 \%$ of the average area is overlooked in the created shapefiles as shown by OE. By analyzing above table, two facts are observable: the building areas are proximately fully reconstructed by the algorithm, both $\mathrm{CE}$ and $\mathrm{OE}$ are less than $6 \%$. As such, the algorithm accomplishes satisfactory results.

Table 5.10 Accuracy evaluation

\begin{tabular}{|c|c|c|c|}
\hline & $\mathrm{OA}$ & $\mathrm{CE}$ & $\mathrm{OE}$ \\
\hline RIM & $101.24 \%$ & $5.72 \%$ & $6.87 \%$ \\
\hline BFG & $96.82 \%$ & $6.87 \%$ & $3.81 \%$ \\
\hline Optometry & $103.09 \%$ & $3.42 \%$ & $6.31 \%$ \\
\hline Average & $100.38 \%$ & $5.34 \%$ & $5.66 \%$ \\
\hline
\end{tabular}

Among the main factors that contribute the inaccuracies of created shapefiles, several aspects need to be examined in more detail.

HRV causes constant obstacle in building roof outline verification, it shadows neighboring buildings partly, which makes it complicated to separate building roof outline points from tree canopies, especially in situations where there is no auxiliary data available like a field map or an aerial image. The east part of the Optometry building suffers distortion of border line and loss of part of border region from strong appearance of HRV. There are some 
remedies accessible to alleviate the impact of HRV, avoiding flight operation during tree bloom season and seeking verification from another form of data in dense HRV areas.

The scanning rate of dataset is $35 \mathrm{kHz}$, which is pretty low sampling rate considering the prevailing scanning rate is more than $200 \mathrm{kHz}$. As a result some elaborate details of building fails to be recorded. Both the BFG and the Optometry buildings have some subtle curves in roof outline design as shown in

Figure 5.36. Omission of such information in the original topographic LiDAR dataset leads to the failure to retrieve proper border lines from algorithm.

Building roof outline regularizing algorithm assumes that all roof edge lines are straight, it works well for the BFG and the Optometry buildings, but introduces problem when obvious curve border line is present in the North East part of the RIM building. The linear substitution of curve line causes part of building failed to be recovered and it is exhibited in Figure $5.36(a)$.

Standard shapefiles are from map library and there are no descriptions about how they are created. Standard shapefiles are assumed to be accurate in this study, however in Figure 5.36, it is observable that all the created shapefiles slide certain extent to south, which leaves gaps between two version of roof outlines in north part for each building. In order to 
trace the origin of the problem, building roof points of the RIM building are extracted and overlaid with standard shapefile. Figure 5.37 shows overlay image of two datasets, as indicated by arrow, there is clearly down shift of original topographic LiDAR raw data compared to standard shapefile, which contributes to both OE's and CE's in Table 5.10. The cause of discrepancy is difficult to locate for lack of necessary information since it may come from the calibration process after the data is collected or from GPS errors during operation. The accuracy level will be further increased if systematic errors are reduced.

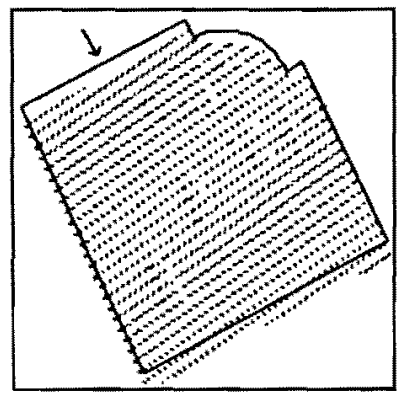

Figure 5.37 Overlay of RIM building roof points from topographic LiDAR data and standard shapefile

\subsection{Chapter Summary}

In this chapter, a complete case of 3D building reconstruction from topographic LiDAR raw data is implemented. The study area is part of UW campus. The experiment consists of five parts. First, raw data is segmented by GRMF-MCMC algorithm proposed in Chapter 4 . Second, filtering process removes HRV points from dataset. Thirdly, building roof edge points are extracted and regularized. In the next step 3D buildings are reconstructed in an 
ArcGIS environment. Finally, an accuracy assessment is conducted, which shows that the whole process achieves promising outcome with low point density topographic LiDAR data alone, 


\section{CONCLUSIONS AND RECOMMENDATIONS}

\subsection{Conclusions}

Rapid and accurate reconstruction of $3 \mathrm{D}$ building models in urban areas remains a challenging task for geomatics community. Topographic LiDAR systems are capable of acquiring 3D information directly over terrain features and become a very active research topic in recent years. In this thesis, a method of 3D building model reconstruction from topographic LiDAR point clouds is presented. Compared with existing approaches, this procedure has two advantages. First, it works on LiDAR point clouds directly without pre-processing or rasterization, which eliminates the loss of spatial information during interpolation step. Second, it functions alone without auxiliary data such as vector maps or GIS data, which makes the approach more versatile.

The proposed approach consists of four steps: building roof detection, roof outline extraction, and regularization, and $3 \mathrm{D}$ building model generation. In the segmentation step, mathematical model resembling the distribution of LiDAR data is established by Gaussian distribution and MRF theory. The MCMC algorithm is utilized to obtain the optimal solution. The building outlines are extracted by the $\mathrm{MCH}$ algorithm. However, the outlines are distorted due the nature of topographic LiDAR systems and further refinement is required. The regularization of the extracted roof outlines is achieved by a modified hierarchical 
regularization algorithm. Finally, the regularized the building roof outlines are input to the ArcGIS software to generate 3D building models.

Among the four aforementioned steps, the building detection is the most critical one and determines the quality of the building models. In this study, a new algorithm called GMRF-MCMC is proposed. The contributions of this algorithm lie in following two perspectives:

1. Topographic LiDAR data can be viewed as a large number of points positioned in 3D co-ordinate system, it is relatively easy and obvious to find solutions pursuing spatial geometry path and this is what most other researchers did in processing topographic LiDAR data. The GMRF-MCMC algorithm takes another route by introducing statistics and probability theory to solve geometry problem. This attempt will benefit other researchers in terms of diversifying mind and developing novel solutions.

2. This algorithm provides user with solution quality control that best matches the application intention. If the application requires fine resolution like what is presented in the Optometry building, parameters are adjustable, and more computing time and memory space are needed as a trade off. If a rough solution is acceptable, then a quick result is achievable in a shorter time and with less cost. This function is desirable especially in handling large volume of data like topographic LiDAR data. 
The accuracy assessment is conducted through a comparison with the standard shapefiles of the buildings. The proposed method detected the full building areas with less than $6 \%$ of commission and omission errors. The results are satisfactory despite the fact that the sample LiDAR data are low accuracy data and the systematic errors exist during data collection.

The approach demonstrated in this thesis provides an efficient and accurate way to reconstruct 3D building models from topographic LiDAR point clouds. The objectives of this study are fulfilled.

\subsection{Recommendations for Future Research}

Although the proposed approach achieves promising results, several aspects for future research still remain.

The GMRF-MCMC algorithm requires human interaction, expertise is necessary in terms of having clear goal and choice of parameters, which causes inconvenience for non-professional users. A full automation algorithm is more user-friendly where the user only needs to choose the accuracy level and the algorithm can estimate best matching category number and parameter combination. Running time is one of the main concerns for this approach, as dataset becomes larger, time consumption will increase. 
The proposed algorithm is point-based algorithm, it takes each point into consideration, and then groups points with analogous properties together. This approach is straight forward but not highly time efficient. If dataset is divided into finite portion of subsets based on certain criteria, it would be more time economic when joining these subsets back together after processing. This area-based method will yield a faster outcome than a point-based method.

During building outline regularization, the algorithm assumes that all the building roof edges are straight. This assumption is straight forward but may introduce discrepancies when handling buildings with complicated footprints. The curve roof edge detection algorithm will reduce omission error.

HRV is one of the main sources of artifact and can significantly deteriorate the preciseness of 3D building models. In topographic LiDAR point clouds, HRV points have multiple echoes, larger elevation differences and distinct intensity values compared with building roof and ground points. These properties can be utilized to separate HRV points from other points, which will greatly increase the accuracy rate, especially in heavy wooded regions. 


\section{REFERENCES}

Alharthy A., J. Bethel, E. M. Mikhail, 2004. Analysis and accuracy assessment of airborne lasers canning system, Proceedings of the 20th ISPRS Congress, Commission II: 114-149.

Arefi, H. and M. Hahn, 2005. A hierarchical procedure for segmentation and classification of airborne LiDAR images, IEEE International; 7(7): 4950-4953.

ASPRS LiDAR Guidelines: Horizontal Accuracy Reporting, 2008. URL: http://www.asprs.org/society/divisions/ppd/standards/Horizontal_Accuracy_Reporting_fo r_ALS_Data.pdf (last date accessed: 10 January, 2008).

ASPRS LiDAR Guidelines : Vertical Accuracy Reporting for LiDAR Data, 2008. URL: http://www.asprs.org/society/committees/ALS/Downloads/Vertical_Accuracy_Reporting _for_ALS_Data.pdf (last date accessed: 15 January, 2008).

Axelsson, P., 2000. DEM Generation from laser scanner data using TIN adaptive models, International Archives of Photogrammetry \& Remote Sensing, 33(B3): 85-92.

Baltsavias, E. P., 1999a. A comparison between photogrammetry and laser scanning, ISPRS Journal of Photogrammetry \& Remote Sensing, 54(2-3): 83-94.

Baltsavias, E. P., 1999b. Airborne laser scanning: basic relations and formulas, ISPRS Journal of Photogrammetry \& Remote Sensing, 54(2-3): 199-214.

Baltsavias, E. P., 1999c. Airborne laser scanning: existing systems and firms and other 
resources, ISPRS Journal of Photogrammetry \& Remote Sensing, 54(2-3): 164-198.

Baltsavias, E.P., E. Favey, A. Bauder, H. Bosch, and M. Pateraki, 2001. Digital surface modelling by airborne laser scanning and digital photogrammetry for glacier monitoring. The Photogrammetric Record, 17 (98): 243-273.

Barber, D., 2006. The LAS Format, Proceedings of the Heritage $3 d$ Workshop, Newcastle, The United Kingdom. URL: http://www.ceg.ncl.ac.uk/heritage3d/downloads/ workshop2006/barber1.pdf (last date accessed: 05 February 2008)

Bouman, A. C., 1995. Markov Random Fields and Stochastic Image Models, Proceeding of IEEE International Conference on Image Processing, Washington D.C., USA.

Brovelli, M., M. Cannata, and U. Longoni, 2002. Managing and processing LiDAR data within GRASS. Proceedings of the Open Source GIS-GRASS users conference, Trento, Italy.

Charaniya, A., R. Manduchi and S. Lodha, 2004. Supervised parameteric classification of aerial LiDAR data, Proceedings of IEEE Workshop on Real-Time 3D Sensors, pp. 25-32. Chen, Q., F. Gong, D. Baldocchi, and G. Xie, 2007. Filtering airborne laser scanning data with morphological methods, Photogrammetric Engineering \& Remote Sensing, 73(2): $175-185$.

Crombaghs, M., R. Brügelmann, E. de Min, 2000. On the adjustment of overlapping strips of laser altimeter height data, International Archive of Photogrammetry and Remote Sensing , $33(3 \mathrm{~A}): 230-237$. 
Dash, J., E. Steinle, R. P. Singh and H. P. Bähr, 2004. Automatic building extraction from laser scanning data: an input tool for disaster management, Advances in Space Research, $33(3): 317-322$.

Deng, F., Z. Zhang and J. Zhang, 2004. Construct 3D city model by multi-sensor data, Proceeding of ISPRS Workshop on Service and Application of Spatial Data Infrastructure, $187-190$.

Dorninger, P. and N. Pfeifer, 2008. A comprehensive automated 3d approach for building extraction, reconstruction, and regularization from airborne laser scanning point clouds, Sensor, 8(11): 7323-7343.

Douglas, D. H. and T. K. Peucker, 1973. Algorithms for the reduction of the number of points required to represent a digitized line or its caricature. Canadian Cartographer, 10(2): $112-122$.

Elberink, S. O. and G. Vosselman, 2006. 3D modeling of topographic objects by fusing 2D maps and LiDAR data, International Archives of Photogrammetry and Remote Sensing, $36(4): 175-184$.

Fan, T., G. Medioni, and R. Nevatia, 1987. Segmented description of 3-D surfaces, IEEE Transactions on Robotics and Automation, 3(6): 527-538.

Flood, M, and B. Gutelius, 1997. Commercial implications of topographic terrain mapping using scanning airborne laser radar, photogrammetric engineering \& remote sensing, Photogrammetric Engineering and Remote Sensing, 63(4): 327-329. 
Flood, M., 2001. Eye safety concerns in ALS mapping, Proceedings of the American Society for Photogrammetry and Remote Sensing Annual Convention, pp. 23-27.

Flood, M., 2002. Product definitions and guidelines for use in specifying ALS deliverables, Photogrammetric Engineering \& Remote Sensing, 68 (12): 785-791.

Fowler, R., 2001. Topographic LiDAR, in Digital Elevation Model Technologies and Applications: The DEM Users Manual, David F. Maune, Editor, The American Society for Photogrammetry andRemote Sensing, Bethesda, Maryland, USA, 539 pages.

GIM International Product Survey: ALS Sensors (2007):21(2). URL: http://www.gim-international.com/productsurvey/id19-Airborne_ALS_Sensors_Fe bruary_,_Volume_,_Issue.html, (last date accessed: 15 March 2008).

Gorte, B., 2002. Segmentation of tin-structured surface models, Symposium on geospatial theory, Processing and Applications, Ottawa, Canada, pp. 278-281.

Haala, N. and K. Brenner 1997. Generation of 3D city models from airborne laser scanning data. Proceedings EARSEL Workshop on LiDAR Remote Sensing on Land and Sea, Tallinn/Estonia, pp. 145-153.

Haala, N., C. Brenner and K.-H. Anders,1998. 3D urban GIS from laser altimeter and 2D map data, International Archives of Photogrammetry and Remote Sensing, 32(3/1):339-346.

Han, S. H., J. H. Lee and K. Y. Yu, 2007. An approach for segmentation of airborne laser point clouds utilizing scan-line characteristics, Electronics and Telecommunications 
Research Institute (ETRI), 29 (5): 641-648.

Han, S. H., J. H. Lee, and K.Y. Yu, 2007. An approach for segmentation of airborne laser point clouds utilizing scan-line characteristics, ETRI Journal, 29 (5): 641-648.

Heesch, D and M. Petrou, 2007. Non-Gibbsian Markov random field models for contextual labeling of structured scenes, Proceedings of British Machine Vision Conference, University of Warwick, UK, pp. 183-192.

Hofmann, A., 2004. Analysis of TIN-structure parameter spaces in airborne laser scanner data for 3-D building model generation, International Archives of Photogrammetry and Remote Sensing, 35(B3):302-307.

Huber, M., W. Schickler, S. Hinz and A. Baumgartner, 2003. Fusion of LiDAR data and aerial imagery for automatic reconstruction of building surfaces, Remote Sensing and Data Fusion over Urban Areas, 2nd GRSS/ISPRS Joint Workshop on International Conference on Image Processing, Washington, D.C., USA, pp. 82-86.

Inside Mac Media, Inc., 2008. URL: http://www.osxfaq.com/man/5/a.out.ws (last date accessed: March 5 2008).

Isenburg, M. and J. Shewchuk, 2008. LAStools: converting, viewing, and compressing LiDAR data in LAS format, URL:http://www.cs.unc.edu/ isenburg/lastools/ (last date accessed: 24 October 2008).

Jenks, G. F., 1989. Geographic logic in line generalization, Cartographica, 26 (1): $27-42$. Jiang, X. and H. Bunke, 1994. Fast segmentation of range images into planar regions by 
scanline grouping. Machine Vision and Applications ,7 (2):115-122.

Jonas, D., and P. Byrne, 2003. Airborne laser scanning: beyond its formative years, URL: http://www.aamhatch.com.au/resources/pdf/publications/technical_papers/Ssc2003_Jona s.pdf / (last date accessed: 29 April 2008).

Jwa, Y., G. Sohn, V. Tao and W. Cho, 2008. An implicit geometric regularization of 3d building shape using airborne LiDAR data, International Archives of Photogrammetry and Remote Sensing, 37 (B3a): 69-76.

Kilian, J., N. Haala, and M. English, 1996. Capture and evaluation of airborne laser scanner data, International Archives of Photogrammetry and Remote Sensing, 31 (B3): 383-388.

Kim, C., M. Ghanma, and A. Habib, 2006. Integration of Photogrammetric and LiDAR data for realistic 3D model generation, first International Workshop on Mobile Geospatial Augmented Reality, Banff, Canada, pp. 135-143.

Kraus, K and N. Pfeifer, 1997. A new method for surface reconstruction from laser scanner data, International Archives of Photogrammetry \& Remote Sensing, 32(3-2W3): 80-86.

Kraus, K. and N. Pfeifer, 1998. Determination of terrain models in wooded areas with airborne laser scanner data, ISPRS Journal of Photogrammetry \& Remote Sensing, 53(4):193-203.

LAS format, 2008. URL: http://www.asprs.org/resources/standards/ALS_format.html (last date accèssed: 10 January 2008).

Lee, H. S and N. H. Younan, 2003. DTM extraction of LiDAR returns via adaptive 
processing, IEEE Transactions On Geoscience and Remote Sensing, 41(9): 2063-2069.

Lee, I. and T. Schenk, 2001. 3d perceptual organization of laser altimetry data, International Archives of Photogrammetry and Remote Sensing, 34:57-65.

Lee, J. H., S. H. Han, Y. G. Byun, K. Y. Yu, and Y. I. Kim, 2007. Building extraction and 3d modeling from airborne laser scanning data, Korean Journal of Remote Sensing, 23(5): $447 \sim 453$.

Lin, S. and $\mathrm{H}$. Wu, 2006. Sweep line based filtering of airborne laser scanning data, Proceedings of SPIE, the International Society for Optical Engineering, 6419: 64191S-64191S-10.

Lohmann, P., 2002. Segmentation and filtering of laser scanner digital surface models, International Archives of Photogrammetry Remote Sensing, 34(2): 311-316.

Maas, H.-G., 1999. Closed solution for the determination of parametric building models from invariant moments of airborne laser scanner data, International Archives of Photogrammetry and Remote Sensing,32(3-2W5):193-199.

Mass, H. G., 2003. Planimetric and height accuracy of airborne laser scanner data: user requirement and system performance, URL:http://www.tu-dresden.de/ipf/photo/publikationen/2003/Maas_PhoWo2003.pdf / (last date accessed: 15 May 2008).

Maune, F. D., 2008. Digital Elevation Model Technologies and Applications: The DEM Users Manual, URL: https:/eserv.asprs.org/eseries/staticcontent/staticpages/1069.htm 
(last date accessed: 20 March 2008).

Morin, K., and E. N. Sheimy, 2002. Post-mission adjustment methods of airborne laser scanning data, International Federation of Surveyors (FIG) XXII International Congress, Washington, D.C. USA, pp. 235-244.

NOAA, 2008. Remote Sensing for Coastal Management, URL:http://www.csc.noaa.gov/crs/rs_apps/sensors/lidar.htm (last date accessed: 14 November 2008).

Optech, 2008. Optech: the LiDAR company, PowerPoint presentation on 12 February 2008, University of Waterloo.

Perez, P., 1998. Markov Random Fields and Images, CWI Quarterly, 11(4): 413-457.

Pfeifer, N., and C. Briese, 2007. Laser scanning - principles and applications, URL: http://publik.tuwien.ac.at/files/pub-geo_1951.pdf/(last date accessed: 20 May 2008).

Pieczynski, w. and A. N. Tebbache, 2000. Pairwise markov random fields and its application in textured images segmentation, Proceedings of 4th IEEE Southwest Symposium on Image Analysis and Interpretation, Austin, USA, pp. 106-110.

Roggero, M., 2001. Airborne laser scanning: clustering in raw data, International Archives of the Photogrammetry and Remote Sensing, 34 (B3/W4): 227-232.

Rönnholm , P., E. Honkavaara, P. Litkey, H. Hyyppä and J. Hyyppä, 2007. Integration of laser scanning and photogrammetry, International Archives of Photogrammetry and Remote Sensing, 36(3/W52):355-362. 
Rottensteiner, F, J. Trinder, S. Clode and K. Kubik, 2003. Building detection using LiDAR data and multi-spectral images, Proceedings of 7th Digital Image Computing: Techniques and Applications, Sydney, Australia, pp. 673-682.

Rottensteiner, F, and C. Briese, 2002. A new method for building extraction in urban areas from high-resolution LiDAR data, Proceedings of the ISPRS Commission III Symposium, Graz, Austria, pp. 295-301.

Samberg, A., 2007. An implementation of the ASPRS LAS standard, International Archives of Photogrammetry and Remote Sensing, 36 (3 / W52):363-372.

Sampath, A and J. Shan, 2007. Building Boundary Tracing and Regularization from Airborne LiDAR Point Clouds, Photogrammetric Engineering and Remote Sensing, 73(7): 805-812.

Sampath, A., and J. Shan, 2004. Urban modeling based on segmentation and regularization of ALS point clouds, International Archive of Photogrammetry and Remote Sensing, 35(B3): 937-941.

Schenk, T., 2001. Modeling and recovering systematic errors in airborne laser scanners, The European Organization for Experimental Photogrammetric Research (OEEPE) Workshop on Airborne Laser scanning and Interferometric SAR for Detailed Digital Elevation Model, pp. 40-48.

Schickler, w. and A. Thorpe, 2001. Surface estimation based on LiDAR, URL:http://www.sanmap.com/Pdfs/Article_SurfaceEstimation.pdf/ (last date accessed: 10 
May 2008).

Schwalbe, E., H. Maas and F. Seidel, 2005. 3D building model generation from airborne laser scanner data using 2D GIS data and orthogonal point cloud projections, ISPRS WG III/3, III/4, V/3 Workshop "Laser scanning 2005", Enschede, the Netherlands, pp. 209-214.

Sentinel Archiving, Inc, 2008. The electromagnetic nature of light. URL: http://www.sentinelarchiving.com/ARTICLES/electromag.htm (last data accessed: 18 January 2008).

Shan, J and A. Sampath, 2005. Urban DEM generation from raw LiDAR data: a labeling algorithm and its performance, Photogrammetric Engineering \& Remote Sensing, 71(2): $217-226$.

Sithole, G. and G, Vosselman, 2004. Experimental comparison of filter algorithms for bare-Earth extraction from airborne laser scanning point clouds, ISPRS Journal of Photogrammetry \& Remote Sensing, 59: 85-101.

Sithole, G. and G, Vosselman, 2005. Filtering of airborne laser scanner data based on segmented point clouds, International Society of Photogrammetry and Remote Sensing, Workshop "Laser scanning 2005", Enschede, the Netherlands, pp. 66-71.

Sithole, G. and G. Vosselman, 2003. Comparison of filtering algorithms, Proceedings of the ISPRS working group III/3 workshop 3-D reconstruction from airborne laser scanner and InSAR data', Dresden, Germany, pp. 240-247.

Sithole, G., 2001. Filtering of laser altimetry data using a slope adaptive filter, International 
Archives of the Photogrammetry and Remote Sensing, 34(3/W4): 203-210.

Sithole, G., 2005. Segmentation and classification of airborne laser scanner data, Publications on Geodesy 59, NCG, Delft.

Steed, A., S. Spinello, B. Croxford and R. Milton, 2004. Data visualization within urban models, Proceedings Theory and Practice of Computer Graphics, IEEE Computer Society, Washington D.C, USA, pp. 9-16.

Straatsma, M.W., and H. Middelkoop, 2006. Airborne laser scanning as a tool for low land floodplain vegetation monitoring, Hydrobiologia, 565:87-103.

Tarsha-Kurdi, F., T. Landes and P. Grussenmeyer, 2007. Joint combination of point cloud and DSM for 3D building reconstruction using airborne laser scanner data, Proceedings of Urban Remote Sensing Joint Event, Paris, France, pp.1-7.

Tarsha-Kurdi, F., T. Landes, P. Grussenmeyer and E. Smigiel, 2006. New approach for automatic detection of buildings in airborne laser scanner data using first echo only, International Archives of Photogrammetry and Remote Sensing, 36(3): 25-30.

ThuyVu, T. and M. Tokunaga, 2002. Designing of wavelet-based processing system for airborne laser scanner segmentation. International Archives of the Photogrammetry and Remote Sensing, 34(5/W3):1682-1777.

TMSI, 2005. The Global Market for ALS Systems and Services, TMS International Ltd., Houston, Texas, $158 \mathrm{p}$.

Tolt, G., U. Söderman and S. Ahlberg, 2007. 3D Urban models from laser radar data, Urban 
Remote Sensing Joint Event, 2007, Paris, France, pp.1-5.

Tovari, D. and N. Pfeifer, 2005. Segmentation based robust interpolation - a new approach to laser data filtering, International Archives of Photogrammetry and Remote Sensing, 33(3/W19): 79-84.

Ung, A., 1996. Retargetable loader, URL:

http://www.itee.uq.edu.au/ cristina/students/david/honoursThesis $96 / \mathrm{bff} . h$ tm (last date accessed: 24 January 2008).

Vosselman, G. and H. Mass, 2001. Adjustment and filtering of raw laser altimetry data, URL:http://www.tu-dresden.de/ipf/photo/publikationen/aeltere/Vosselmann_Maas_OEE PEStockholm2001.pdf / (last data accessed: 15 April 2008).

Vosselman, G. and S. Dijkman, 2001. 3D building model reconstruction from point clouds and ground plans, International Archives of Photogrammetry and Remote Sensing, $36(3 / W 4): 37-43$.

Vosselman, G., 2000. Slope based filtering of laser altimetry data, International Archives of Photogrammetry and Remote Sensing, 33(B4): 958-964.

Vosselman, G.,2002. Fusion of laser scanning data, maps, and aerial photographs for building reconstruction, Geoscience and Remote Sensing Symposium, 2002. IGARSS '02. 2002 IEEE International, 1:85-88.

Wang, M. and Y.-H. Tseng, 2004. LiDAR data segmentation and classification based on octree structure. International Archives of the Photogrammetry and Remote Sensing, 
35(B3): 308-313.

Webster, T. L., and G. Dias, 2006. An automated GIS procedure for comparing GPS and proximal ALS elevations, Computers \& Geosciences 32: 713-726.

Webster, T.L., D. L. Forbes, S. Dickie, 2004. Using topographic ALS to map flood risk from storm-surge events from Charlottetown, Prince Edward Island. Canadian Journal of Remote Sensing 30 (1): 64-76.

Wehr, A., and U. Lohr, 1999. Airborne laser scanning-an introduction and overview, ISPRS Journal of Photogrammetry \& Remote Sensing, 54(2-3): 68-82.

Wehr, A., U. Lohr, and E. Baltsavias, 1999. Theme issue on airborne laser scanning, ISPRS Journal of Photogrammetry \& Remote Sensing, 54(2-3):.61-63.

Weidner, U. and W. Förstner, 1995. Towards automatic building extraction from high resolution digital elevation models, ISPRS Journal of Photogrammetry \& Remote Sensing, $50(4): 38-49$.

Woo, H., E. Kang, S. Wang and K. H. Lee, 2002. A new segmentation method for point cloud data, International Journal of Machine Tools \& Manufacture, 42: 167-178.

Zhang, X. and J. Liu, 2004. Analysis of systematic error influences on accuracy of airborne laser scanning altimetry, Geo-spatial Information, 7(3): 218-224.

Zhang; K., S. -C. Chen, D. Whitman, M. -L. Shyu, J. Yan and C. Zhang, 2003. A progressive morphological filter for removing nonground measurements from airborne LiDAR data, IEEE Transactions on Geoscience and Remote Sensing, 41(4): 872-882. 
Zhang, Y., 2000. Markov random field theory.

URL:http://www.fmrib.ox.ac.uk/analysis/techrep/tr00yzl/tr00yzl/node4.html (last date accessed: 2 October 2008). 